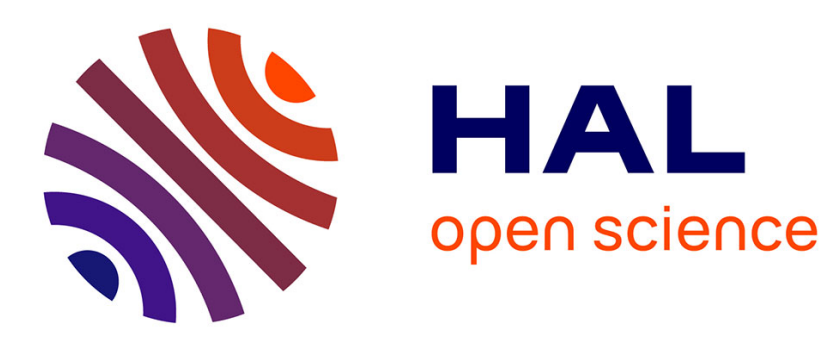

\title{
On Devising Various Alarm Systems for Insurance Companies
}

\author{
Marie Kratz, Shubhabrata Das
}

\section{To cite this version:}

Marie Kratz, Shubhabrata Das. On Devising Various Alarm Systems for Insurance Companies. 2010. hal-00572546

\section{HAL Id: hal-00572546 \\ https://essec.hal.science/hal-00572546}

Submitted on 7 Mar 2011

HAL is a multi-disciplinary open access archive for the deposit and dissemination of scientific research documents, whether they are published or not. The documents may come from teaching and research institutions in France or abroad, or from public or private research centers.
L'archive ouverte pluridisciplinaire HAL, est destinée au dépôt et à la diffusion de documents scientifiques de niveau recherche, publiés ou non, émanant des établissements d'enseignement et de recherche français ou étrangers, des laboratoires publics ou privés. 


\title{
On Devising Various Alarm Systems for Insurance Companies
}

\author{
Research Center \\ ESSEC Working Paper 10008 \\ December 2010
}

Shubhabrata Das

Marie Kratz 


\title{
On Devising Various Alarm Systems for Insurance Companies
}

\author{
Shubhabrata Das * and Marie Kratz $^{\dagger} \ddagger$
}

\begin{abstract}
One possible way of risk management for an insurance company is to develop an early and appropriate alarm system before the possible ruin. The ruin is defined through the status of the aggregate risk process, which in turn is determined by premium accumulation as well as claim settlement out-go for the insurance company. The main purpose of this work is to design an effective alarm system, i.e. to define alarm times and to recommend augmentation of capital of suitable magnitude at those points to prevent or reduce the chance of ruin. In the three different methods outlined in this work, the alarms are signaled on the basis of the past history of the risk process and/or properties of claim distribution. Depending on the method adopted, the alarm time can be a random one or a fixed parameter of the claim distribution (and premium function). The focus of this work is on devising a sequence of alarms, which are indeed fixed parameters based on characteristics of the risk process. To draw a fair measure of effectiveness of alarm system(s), comparison is drawn between a process equipped with an alarm system, with capital being added at the sound of every alarm, and the corresponding process without any alarm system but an equivalently higher initial capital. Detailed analytical results are obtained for general processes and this is backed up simulated performances when the loss severity has exponential, or Pareto or discrete logarithmic distribution. The formulation is eventually intended to be applied and extended for devising alarm system for reinsurance contracts.
\end{abstract}

AMS classification. 91B30, 60K30 Keywords: alarm system, capital accumulation function, efficiency, quantitative risk management, risk process, ruin probability.

\section{Introduction, Notation and Literature Review}

\subsection{Introduction and Overview}

This work develops an early and appropriate alarm system for an insurance institution before its possible ruin based on pattern of premium collection and demands for claim settlement. While keeping a very high initial capital may avoid ruin for the insurance company, it is neither desired by most companies because of obvious investment concerns, nor is feasible at times. An effective alarm system opens the door for an alternate strategy based on ruin theory by opting for less initial capital and topping it up when really necessary.

The work may be applied from two perspectives. On one hand, it can be viewed from a regulatory perspective to provide guidance for regulatory intervention, without compromising the capacity of a company to survive. On the other hand, companies may use it to design good triggers for obtaining contingent capital from banks. The alarm system could serve in structuring such contract to improve capital management.

${ }^{*}$ IIM Bangalore, India

${ }^{\dagger}$ ESSEC Business School Paris, France

${ }^{\ddagger}$ MAP5 (UMR8145, Univ. Paris Descartes) 
Alarm systems have been developed in different contexts in the literature (viz. [14], [7], [17] and references therein), while capital reserving or capital allocation have been addressed in many articles (viz. [3], [13], and references therein). In particular, in [13] Kaishev et al. showed numerically that two capital accumulation functions, one linear and the other piecewise linear with one jump at some instances, would lead to equal chances of survival and also equal accumulated risk capital at the end of the considered time interval. The approach in the present work, with the introduction of a new alarm system, is fundamentally different, even though the broad concern is similar, i.e. to reduce the initial capital without compromising on the survival probability.

The basic idea behind our proposed notion of alarm is as follows. An alarm is sounded at a juncture when the probability of ruin (in absence of any intervention) within a specified future time period is high enough. While few variations in defining the alarm time have been explored in this work, in the main approach we find it more appropriate when the above probability is set in terms of a conditional event given survival up to the alarm time. In addition, we require that the chance of no ruin before the alarm should be sufficiently high. The natural extension of single alarm when adding capital at the sound of each alarm leads to the definition of an alarm system consisting of successive alarms. This system constitutes an alternate strategy for having to put up an excessive initial capital to avoid ruin.

Note that this strategy does not interfere with the Value-at-Risk approach (or any tail approach) applied by insurance companies as mandated by the Solvency regulation. It just means that the capital may also be adjusted on a regular basis (e.g. every quarter) for the risk adjusted capital to be higher than the capital required by Solvency.

For fair evaluation of effectiveness of our strategy, the proposed alarm system is pitted against a default no-alarm system equipped with equivalent higher initial capital. We compare the survival probabilities under the alternatives. In the longer run, the alarm system is expected to win, as is indeed confirmed by our study. In the shorter run, the alarm system may be preferred even if the chance of survival under this is marginally worse. With that being the objective, we focus on analytical as well as numerical evaluations of the comparative survival probabilities under the two systems.

To illustrate our method, we consider a simple linear accumulation model. So the adjustment of capital as mandated by Solvency Rules, might be easily accommodated in the setup using a stepwise linear accumulation function. This simple framework is neither a prerequisite for the proposed formulation and nor the essence of our work. It would be valid irrespective of whether the claim amounts are independent, or identically distributed, or otherwise. Thus our method has the advantage of being simple and adaptable to any model. If the stochastic nature of the risk process is completely known, as assumed in this work, the alarm times are naturally fixed known parameters, depending on various parameters of the underlying risk process. In practice, the proposed mechanism may be embedded into an adaptive scheme where additional information regarding the risk process in terms of claims would be recursively/progressively utilized to lead to a suitable random alarm system that draws on empirical information on claims.

The paper is organized as follows. In Section 1.2, we introduce the basic notation and framework of this paper while in Section 1.3 we report key results from the existing literature. The main approach of this paper is detailed in Section 2, where we also present various related computational results. The computational issues as well as the development of the entire alarm system are also included in this Section. The effectiveness of alarm systems and comparison across the different options including that of not adopting any alarm system is critical; this is taken up in Section 3. Two alternative approaches for defining the alarm times are described in the next section. In Section 4.1, we present a simple approach of defining alarm based on just the severity of claims. As opposed to these two non-random definitions of alarm, we also consider a random alarm time in Section 4.2. Section 4.3 briefly outlines possible approaches to defining alarms for reinsurance contracts. Finally Section 5 concludes with some remarks and observations. 


\subsection{Notations and Framework}

We follow the classical ruin theory framework and adopt the Cramér Lundberg model to present our general approach. To keep the framework as general as possible, most of the definitions and results will be given in terms of the ruin probability, and consequently the results on (joint) c.d.f. of ruin time for dependent or independent claims can be found in the existing literature. However, for comparing our formal results with simulations, we consider simpler setup with i.i.d. simulated claims, since the aim is only to illustrate our method, although it may be extended for dependent claims using e.g. copula methods. Much of the analysis of this paper can be carried over in a straightforward way to more general Lévy processes and premium rates.

Let us assume that the $i$-th claim is of magnitude (severity) $X_{i}$ and happens at time $T_{i}$, for $i \geq 1$. By default, we assume that $X_{i}$ 's are i.i.d. with distribution function $F$ and mean $\mu$. We also assume that the inter-arrival times $W_{k}=T_{k}-T_{k-1}$ are i.i.d. and also independent of the $X_{i}$ 's; a common model for the distribution of the $W_{i}$ 's is exponential with parameter $\lambda_{W}$ and in that case the claim process is said to follow a Poisson process. For most part we consider such Poisson process but with various different claim distributions $F$.

Let $T_{0}=0$. Let $\left(N_{t} ; t \geq 0\right)$ be the counting process $N_{t}=\sup \left\{k \geq 1: T_{k} \leq t\right\}$ corresponding to the Poisson process with intensity $\lambda>0$, independent of the $X_{i}$ 's. Aggregated claims $\left(S_{t} ; t \geq 0\right)$ are defined by $S_{t}=\sum_{i=1}^{N_{t}} X_{i}$. Consider the risk (or surplus) process $\left(V_{t}^{u}, t \geq 0\right)$ given by

$$
V_{t}^{u}=u_{t}+p_{t}-S_{t}=u_{t}-R_{t},
$$

where $u_{t}$ denotes the capital function at time $t$ and the premium rate is linear, viz. $p_{t}=c t$ and the net outgo (without taking capital into account, i.e. aggregate claims less premium collected) process is given by $R_{t}=S_{t}-p_{t}=\sum_{i=1}^{N_{t}} Z_{i}$ with $Z_{i}:=X_{i}-c W_{i}$ (being i.i.d. when so are $X_{i}$ ). Note that while $R_{t}$ is a stochastic process, the capital process $u_{t}$ is non-random and at the discretion of the company. Indeed, one of the key objectives of this work can be restated as the determination of $u_{t}$ given the knowledge of parameters of $R_{t}$. If the decision is to start with only an initial capital $u_{0}=u$ and not to make any further addition, then $u_{t}=u$ for all $t \geq 0$; in such a case, we may denote the risk process, equivalently by $V_{t}^{u}$. This is indeed the starting framework or the benchmark. The ruin time of such a risk process is then formally defined as

$$
T(u)=\inf \left\{t>0: V_{t}^{u}<0\right\}=\inf \left\{t>0: R_{t}>u\right\},
$$

with $T(u)=\infty$ if there is no ruin. Note that while in practice one may wish to define ruin as the first time instance when $V_{t}^{u}$ goes below a level $L$ (other than 0 ), it would take a trivial adjustment in the approach adopted here to carry forward the method. Consequently, in this work, we stick to $L=0$.

Note that for independent claims, $\left(R_{t}\right)$ is a compound Poisson process (Lévy process) and that the c.d.f. $F_{Z}$ of $Z_{i}$ is given in terms of $F$ by

$$
F_{Z}(x)=e^{\lambda x / c} \int_{x}^{\infty} F(z) e^{-\lambda z / c} d z
$$

Recall that for the classical model, the Net Profit Condition (NPC) is given by: $c>\lambda \mu$, i.e. the premium income should exceed expected claim payments. Introducing the premium loading factor $\theta$, we can write $c$ as

$$
c=(1+\theta) \lambda \mu
$$

so the NPC is equivalent to $\theta>0$.

Remark: If $\theta>0$ is small (large), it reflects heavy (light) traffic condition. It is, of course, safer for the company to have the NPC satisfied; but, in practice it may not always be possible. 
An important group of examples of such situations are the heavy-tail severity distributions having infinite mean. Naturally the NPC is violated in such conditions with $\theta$ being -1 . Consequently we strive for formulation and general results that do not depend on the NPC and in many of our computational experiments, the NPC is violated.

Remark: Note that while flexibility in the choice of the initial capital $u$ is an integral part of this work, in the given instance we are concerned with a fixed value for $u$ and not in the asymptotic behaviour with $u \rightarrow \infty$, unlike most related literature. The justification for such asymptotic consideration ([16]) is usually given in terms of avoiding the unit value for the infinite horizon ruin probability. However, not only a fixed initial capital is practical, it is perhaps inevitable (and hence acceptable) that any system will eventually ruin if not interrupted/not intervened at some finite time, however distant it might me. It is also from this consideration that we do not insist on NPC in the present work under all circumstances.

Let $T(a, u)$ denote the ruin time of the risk process $\left(V_{t}^{u} ; t \geq a\right)$ with capital $u$ at time $a \geq 0$ : i.e.

$$
T(a, u)=\inf \left\{t>a: V_{t}^{u}<0\right\}=\inf \left\{t>a: R_{t}>u\right\} .
$$

Set $T(u) \equiv T(0, u)$. The infinite horizon ruin probability with capital $u$ at time $a$ is denoted by:

$$
\psi_{a}(u):=P(T(a, u)<\infty)=P\left(\inf _{t>a} V_{t}^{u}<0\right)=P\left(\sup _{t>a} R_{t}>u\right),
$$

and the corresponding finite horizon ruin probability, which is the distribution function of the r.v. $T(a, u)$, by

$$
\psi_{a}(u, t):=P(T(a, u) \leq t)=P\left(\sup _{a<s \leq t} R_{s}>u\right)
$$

to simplify the notation, we set

$$
\psi_{0}(u)=\psi(u) \quad \text { and } \quad \psi_{0}(u, t)=\psi(u, t)
$$

and also

$$
\bar{\psi}_{a}(u, t)=1-\psi_{a}(u, t) .
$$

Let us introduce the conditional ruin probabilities in infinite and finite times given some event $B$ :

$$
\psi_{a}(u \mid B):=P[T(a, u)<\infty \mid B]=P\left[\inf _{t>a} V_{t}^{u}<0 \mid B\right]=P\left[\sup _{t>a} R_{t}>u \mid B\right]
$$

and

$$
\psi_{a}(u, t \mid B):=P(T(a, u) \in(0, t] \mid B)=P\left[\sup _{a \leq s \leq t} R_{s}>u \mid B\right] .
$$

For $B$ with $P(B=0)$, as would be the case if $B=(Z=z)$ for any continuous random variable $Z$, the above conditional probabilities should be naturally understood, namely as conditional expectations:

$$
\psi_{a}(u, t \mid(Z=z))=E\left[1_{\left(\sup _{a \leq s \leq t} R_{s}>u\right)} \mid Z\right](\omega), \quad \forall \omega \in(Z=z) ;
$$

however, for simplicity, we stick to the notation of conditional probability even in this case throughout the paper.

Notice that, $a$ being fixed, $\psi_{a}(u, t)$ is a non-decreasing function of the time $t$ at given $u$, and is a non-increasing function of the capital $u$ at given $t$. 
Introducing the notation $F_{R_{a}}$ for the cumulative distribution function of $R_{a}$, for a given time $a>0$, we can write

$$
\begin{aligned}
\bar{\psi}(u) & =P\left[\sup _{t>0} R_{t} \leq u\right] \\
& =P\left[\sup _{t>a} R_{t} \leq u, \quad \sup _{0<t \leq a} R_{t} \leq u\right] \\
& =\int_{-\infty}^{u} P\left[\sup _{t>a} R_{t} \leq u, \quad \sup _{0<t \leq a} R_{t} \leq u \quad \mid \quad R_{a}=x\right] d F_{R_{a}}(x) \\
& =\int_{-\infty}^{u} P\left[\sup _{t>a} R_{t}-R_{a} \leq u-x, \sup _{0<t \leq a} R_{t} \leq u \quad \mid \quad R_{a}=x\right] d F_{R_{a}}(x) \\
& =\int_{-\infty}^{u} P\left[\sup _{t>a} R_{t-a} \leq u-x\right] P\left[\sup _{0<t \leq a} R_{t} \leq u \quad \mid \quad R_{a}=x\right] d F_{R_{a}}(x),
\end{aligned}
$$

since $\left(R_{t}\right)$ has independent and stationary increments, in particular, $R_{t}-R_{a}$ is independent of $\sigma\left\{R_{s}: 0<s \leq a\right\}$ and $R_{t}-R_{a} \stackrel{d}{=} R_{t-a}$. This implies:

$$
\bar{\psi}(u)=\int_{-\infty}^{u} \bar{\psi}(u-x) \bar{\psi}\left(u, a \mid R_{a}=x\right) d F_{R_{a}}(x) .
$$

A lower bound for $\bar{\psi}(u)$ can then be deduced, using that $\psi(u)$ is a non-increasing function of $u$, as

$$
\begin{aligned}
\bar{\psi}(u) & \geq \bar{\psi}(0) \int_{-\infty}^{u} \bar{\psi}\left(u, a \mid R_{a}=x\right) d F_{R_{a}}(x) \\
& =\bar{\psi}(0) \bar{\psi}(u, a) .
\end{aligned}
$$

Looking at finite time ruin probabilities, we can proceed in the same way and obtain for $t>a$ (the bound as given in (6) holding good trivially otherwise)

$$
\begin{aligned}
\bar{\psi}(u, t) & =P\left[\sup _{0<s \leq t} R_{s} \leq u\right]=P\left[\sup _{a<s \leq t} R_{s} \leq u, \sup _{0<s \leq a} R_{s} \leq u\right] \\
& =\int_{-\infty}^{u} \bar{\psi}(u-x, t-a) \bar{\psi}\left(u, a \mid R_{a}=x\right) d F_{R_{a}}(x) \\
& \geq \bar{\psi}(0, t-a) \bar{\psi}(u, a) .
\end{aligned}
$$

Notice that the infinite time ruin probabilities can be deduced from the finite ones when taking $t \rightarrow \infty$.

Finally, defining the $\sigma$-field $\sigma_{a}:=\sigma\left\{R_{s} ; s \leq a\right\}$, we obtain for any time $t \in(a, \infty]$, for any element $A$ of $\sigma_{a}$, and for any $u$ and almost all $x$,

$$
\begin{aligned}
\psi_{a}\left(u, t \mid A \cap\left(R_{a}=x\right)\right) & =P\left[\sup _{a<s \leq t} R_{s}>u \mid A \cap\left(R_{a}=x\right)\right] \\
& =P\left[\sup _{a<s \leq t} R_{s}-R_{a}>u-x \mid A \cap\left(R_{a}=x\right)\right] \\
\text { (by independence) } & =P\left[\sup _{a<s \leq t} R_{s}-R_{a}>u-x\right] \\
\text { (by stationarity) } & =P\left[\sup _{a<s \leq t} R_{s-a}>u-x\right] \\
& =\psi(u-x, t-a) .
\end{aligned}
$$

In particular, taking $t \rightarrow \infty$ in (7), we get for any $A \in \sigma_{a}$ :

$$
\psi_{a}\left(u \mid A \cap\left(R_{a}=x\right)\right)=\psi(u-x) .
$$




\subsection{Key relevant results from the existing Literature}

Distributions of ruin time have been explored extensively in the literature; for important representative literature since 2000, see [2], [5], [6], [9], [10], [12], [16]. The alarm times we will define in this paper are simply a parameter of the ruin time distribution, therefore we will attempt to calculate them using such characterizations of ruin-time. From broad considerations, ruin time distributions depend on whether the claims distributions are continuous or discrete, but also on the characterization of the claims tail distributions. Our choices of examples reflect that in attempt to cover broad perspectives. Let us now recall, for the sake of completeness and to help the reading, some exact evaluations of ruin probabilities that can be found in the literature.

\section{- Exponential i.i.d. claims}

It is one of the few cases where ruin probabilities can be explicitly computed. When considering a light-tailed (i.i.d.) claim distributions in the form of exponential distribution with parameter $\rho>0$, the ultimate ruin probability is given, under the NPC, by

$$
\psi(u)=\frac{1}{1+\theta} e^{-\frac{\rho \theta}{1+\theta} u}
$$

with $\theta$ the loading premium factor, which corresponds to the Lundberg's bound up to the multiple factor $(1+\theta)^{-1}$.

The ruin time c.d.f. $\psi(u,$.$) can also be explicitely/analytically computed for exponential claims$ (see e.g. $\S I V$ in [2], or $\S 8$ in [5] when expressing the Bessel function as a series instead of an integral) according to

$$
\psi(u, t)=\psi(u)-\frac{1}{\pi} \int_{0}^{\pi} \frac{f_{u}(t, x) g_{u}(x)}{h(x)} d x
$$

where

$$
\begin{aligned}
f_{u}(t, x)=f_{\rho, \theta, u}(t, x) & =\frac{1}{1+\theta} \exp \left\{\frac{\rho(u+2 t)}{\sqrt{1+\theta}} \cos x-\rho\left(u+\frac{2+\theta}{1+\theta} t\right)\right\}, \\
g_{u}(x)=g_{\rho, \theta, u}(x) & =\cos \left(\frac{\rho u}{\sqrt{1+\theta}} \sin x\right)-\cos \left(\frac{\rho u}{\sqrt{1+\theta}} \sin x+2 x\right), \\
h(x)=h_{\theta}(x) & =\frac{2+\theta}{1+\theta}-\frac{2}{\sqrt{1+\theta}} \cos x .
\end{aligned}
$$

- Exact evaluation of finite time survival probability for any claim (Ignatov et al.)

If $f_{X}$ denotes the joint density function for continuous claims $X$, the finite time survival probability is given by (see [9], Theorem 1)

$$
\begin{array}{r}
\bar{\psi}(u, t)=e^{-\lambda t}\left(1+\sum_{k=1}^{\infty} \lambda^{k} \int_{0}^{u+p(t)} d y_{1} \int_{y_{1}}^{u+p(t)} d y_{2} \ldots \int_{y_{k-1}}^{u+p(t)} A_{k}\left(t ; \nu_{y_{1}}, \ldots, \nu_{y_{k}}\right)\right. \\
\left.f_{X}\left(y_{1}, y_{2}-y_{1}, \ldots, y_{k}-y_{k-1}\right) d y_{k}\right)
\end{array}
$$

where $A_{k}\left(t ; \kappa_{1}, \ldots, \kappa_{k}\right), k \geq 1$, are the classical Appell polynomials $A_{k}(t)$ of degree $k$ with a coefficient in front of $t^{k}$ equal to $1 / k$ !, defined for $k \geq 1$ by $A_{0}(t)=1, A_{k}^{\prime}(t)=A_{k-1}(t)$ and $A_{k}\left(\kappa_{k}\right)=0$, and for $i \geq 0, \nu_{i}=\inf \{t: u+p(t) \geq i\}$. 
For discrete claims, it is given by (see [11])

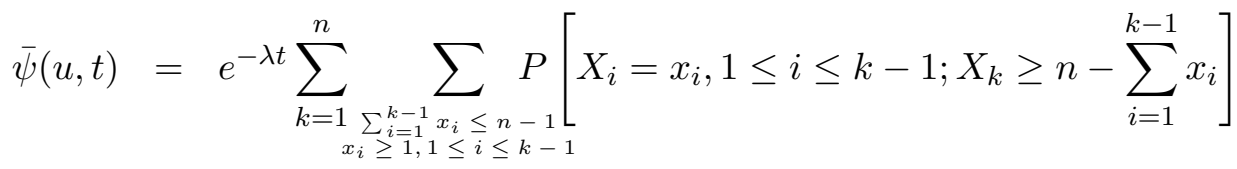

$$
\begin{aligned}
& \sum_{j=0}^{k-1}(-1)^{j} b_{j}\left(z_{1}, \ldots z_{j}\right) \lambda^{j} \sum_{m=1}^{k-j-1}(\lambda t)^{m} / m !
\end{aligned}
$$

where $n$ is the integer part of $(1+u+p(t))$, and for $j \geq 1, z_{j}=\nu_{\sum_{i=1}^{j} x_{i}}$,

$b_{j}\left(z_{1}, \ldots z_{j}\right)=\sum_{i=0}^{j-1} \frac{(-1)^{j+i+1}}{(j-i) !} z_{j}^{j-i} b_{i}\left(z_{1}, \ldots, z_{i}\right)$, with $b_{0}()=$.1 and $b_{1}$ the identity function.

\section{Alarm System Based on Probability of Impending Ruin}

\subsection{Defining Alarm Time}

Here we propose a direct method of devising alarm based on two intuitive requirements of the alarm time, viz.

- at the alarm time, the chance of ruin in not so distant future is substantial if no remedial action is taken;

- the chance of the system getting ruined before this (alarm) time is minimal.

The formal definition, included below, puts these requirements objectively. Although we define two other approaches in Section 4.2 and Section 4.1, this is the main approach adopted in the present work.

Definition. Given specified probabilities $1-\alpha$ and $1-\beta$ and future (lead) time window $d$ and initial capital $u$ (to be chosen by the company), we define the alarm time $A=A(\alpha, \beta, d, u)$ as:

$$
A=\inf \{s>0: P[T(u) \leq s+d \mid T(u)>s] \geq 1-\alpha \text { and } P[T(u)>s] \geq 1-\beta\} .
$$

We have

$$
P[T(u) \leq s+d \mid T(u)>s] \geq 1-\alpha \Leftrightarrow \alpha \bar{\psi}(u, s)-\bar{\psi}(u, s+d) \geq 0
$$

Hence to identify the alarm time $A$, for given $d$ and $\alpha$, we look for the first time $s>0$ satisfying

$$
\begin{gathered}
\qquad \alpha \bar{\psi}(u, s)-\bar{\psi}(u, s+d) \geq 0, \quad \text { and } \bar{\psi}(u, s) \geq 1-\beta, \\
\text { or equivalently } A=\inf _{s>0}\left\{s: \bar{\psi}(u, s) \geq \max \left(1-\beta, \frac{1}{\alpha} \bar{\psi}(u, s+d)\right)\right\} .
\end{gathered}
$$

Remark: Choice of $\alpha, \beta, d: \beta$ in the above specification requirement needs to be small ensuring that the chance of system getting ruined before the sound of alarm is insignificant. On the other hand, $\alpha$ should be (only) moderately small to ensure that the threat of ruin is realistic enough to warrant a remedial action. To emphasize, if $\alpha=0.4$, there is a $60 \%$ chance of ruin in the given future window, which is bad enough for one to consider options available and one need not (actually should not) have to wait for a situation where this chance is very close to 1 (which would be the case, if we were to demand say $\alpha=0.01$ ). The time window $d$ should be moderate, since a 'large' value would imply that the ruin is far from being imminent (and hence perhaps the threat is not 
very serious in that sense), while a small value would give very little opportunity for the remedial actions to take any effect. While in practical situations these choices would be somewhat subjective and/or depending on other problem specific elements, the choice of $\alpha$ and $d$ would be inter-related. We also note that the alarm time, so defined, is actually non-random. We also remark that an alternate formulation of the alarm time could be considered in terms probabilities of joint events as opposed to the the conditional probability as above. We may also note that a simply consequence of our definition of alarm time $A$ is:

$$
P[A<T(u)<A+d] \geq(1-\alpha)(1-\beta) .
$$

As an analytical example let us briefly consider the simple case when the claim severity has an exponential distribution. To find the alarm time $A$ as defined in (12), we assume that $\theta$ does not belong to a neighborhood of 0 for the definition of $A$ to be relevant.

Since $f_{u}(s+d, x)=f_{u}(s, x) \exp \left\{\frac{2(\sqrt{1+\theta}-1) \cos x-\theta}{1+\theta} \rho d\right\}$, we have

$$
f_{u}(s+d, x) \sim f_{u}(s, x) e^{-\frac{\rho \theta}{1+\theta}(1-\cos x) d},
$$

in particular for $\theta<1$. $A$ is then the infimum solution (over $s>0$ ) of the following system:

$$
(S)\left\{\begin{array}{l}
\frac{1}{\pi} \int_{0}^{\pi} \frac{g_{u}(x)}{h(x)} f_{u}(s, x) d x \geq \frac{1}{1+\theta} e^{-\frac{\rho \theta}{1+\theta} u}-\beta \\
\frac{1}{\pi} \int_{0}^{\pi} \frac{g_{u}(x)}{h(x)}\left(\alpha-\exp \left\{\frac{2(\sqrt{1+\theta}-1) \cos x-\theta}{1+\theta} \rho d\right\}\right) f_{u}(s, x) d x \geq \\
\quad(1-\alpha)\left(1-\frac{1}{1+\theta} e^{-\frac{\rho \theta}{1+\theta} u}\right)
\end{array}\right.
$$

and can be found numerically via e.g. Matlab, given the parameters $\rho, \theta, \alpha, \beta$ and $u$. Note that if $\theta$ belongs to a neighborhood of 0 (known as heavy traffic condition), then $f_{u}(s+d, x) \underset{\theta \rightarrow 0}{\sim} f_{u}(s, x)$, hence $\psi(u, s+d) \underset{\theta \rightarrow 0}{\sim} \psi(u, s)$ which makes the definition of $A$ irrelevant.

\subsection{Numerical Illustrations via Simulation}

We undertake simulation studies to better understand the alarm time defined in (12). In particular, the goal is to understand how the alarm time varies with the parameters $\alpha, \beta$ and $d$, also how it depends on the initial capital. To cover discrete and continuous distributions with different tails, heavy vs. light, for claim severities (density $f(\cdot)$ ), we take three examples in the form of exponential, Pareto and (discrete) logarithm distributions. In all these examples, occurrence of claims are assumed to constitute a Poisson process, i.e. the time between successive claims follows an exponential distribution $(\lambda)$; also the risk process entails an initial capital $u_{0}$ and a linear premium function $p_{t}=c t$. All the reported results are based on simulation run of 100000 carried in $\mathrm{R}$.

Example 1 (exponential): Let us first consider a simple homogeneous Poisson claim process with inter-arrival of claims following an exponential distribution with parameter $\lambda=20$ and also the claim severities being i.i.d. exponentially distributed with $\rho=0.5$, with an initial capital of $u_{0}=15$ and a linear premium function of $p_{t}=25 t$. Note that the NPC condition is not satisfied in this case with $\theta$ of (3) being equal to -0.375 , thus implying that the process ruins in finite time with probability 1 . Indeed, we find the distribution of ruin time to be finitely supported with a mode of 0.41 and the ruin time density is shown in Figure 1. 
Figure 1: Density of Ruin time in Example 1

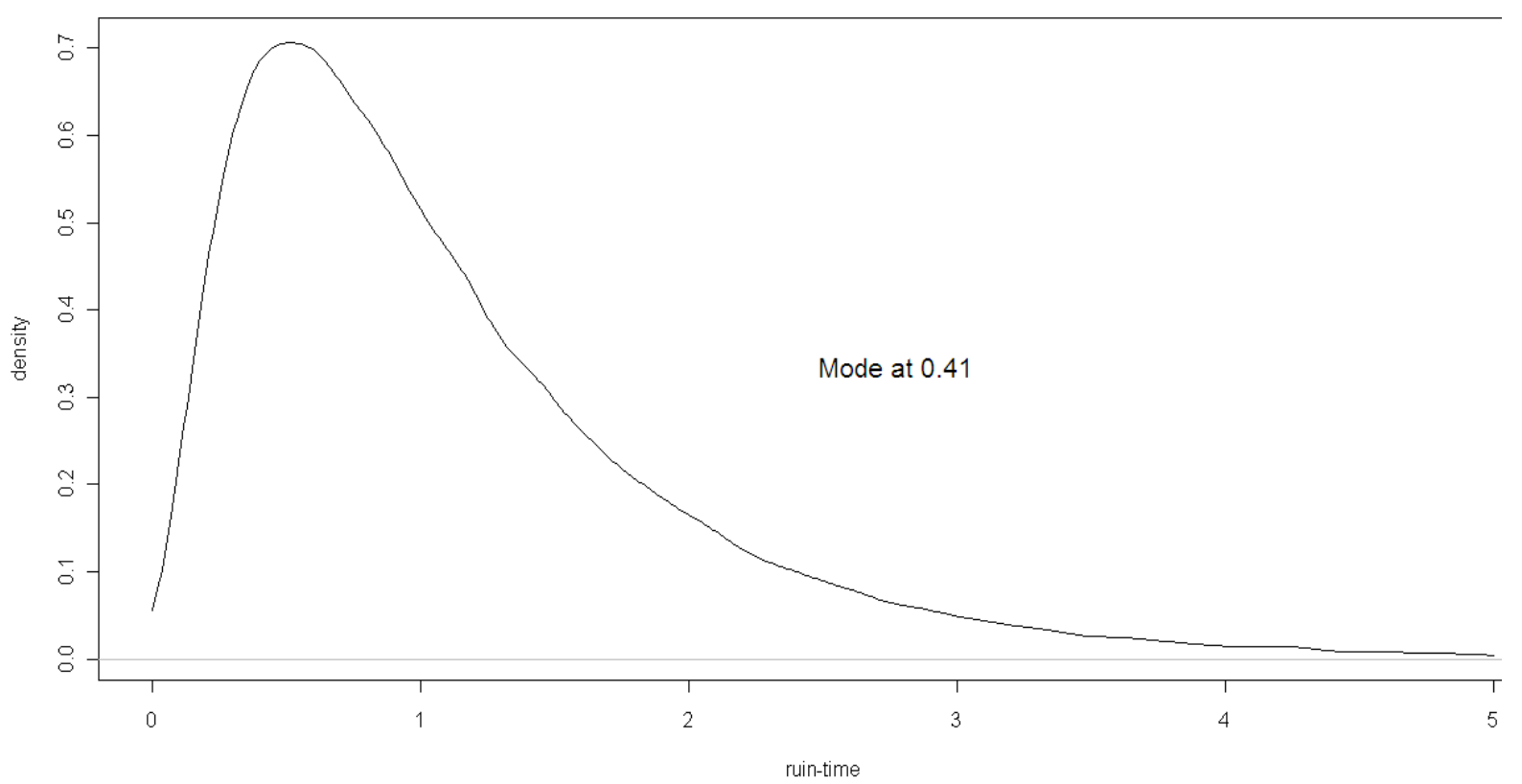

Example 2: (Pareto) We now consider the claims to come from a heavier tail Pareto distribution with infinite mean, having a density function of the form:

$$
f(x)=\frac{\kappa \rho^{\kappa}}{(\rho+x)^{\kappa+1}}, \quad \text { for paramer choice of } \rho=1 \text { and } \kappa=0.95 .
$$

The inter-arrival of claims are again assumed to follow an exponential distribution with parameter $\lambda=20$. The risk process is generated for an initial capital of $u_{0}=50$ and a linear premium function of $p_{t}=40 t$. Ruin time turns out to be finitely supported with a mode of 0.43 with the (kernel) density being shown in Figure 2.

Figure 2: Density of Ruin time in Example 2

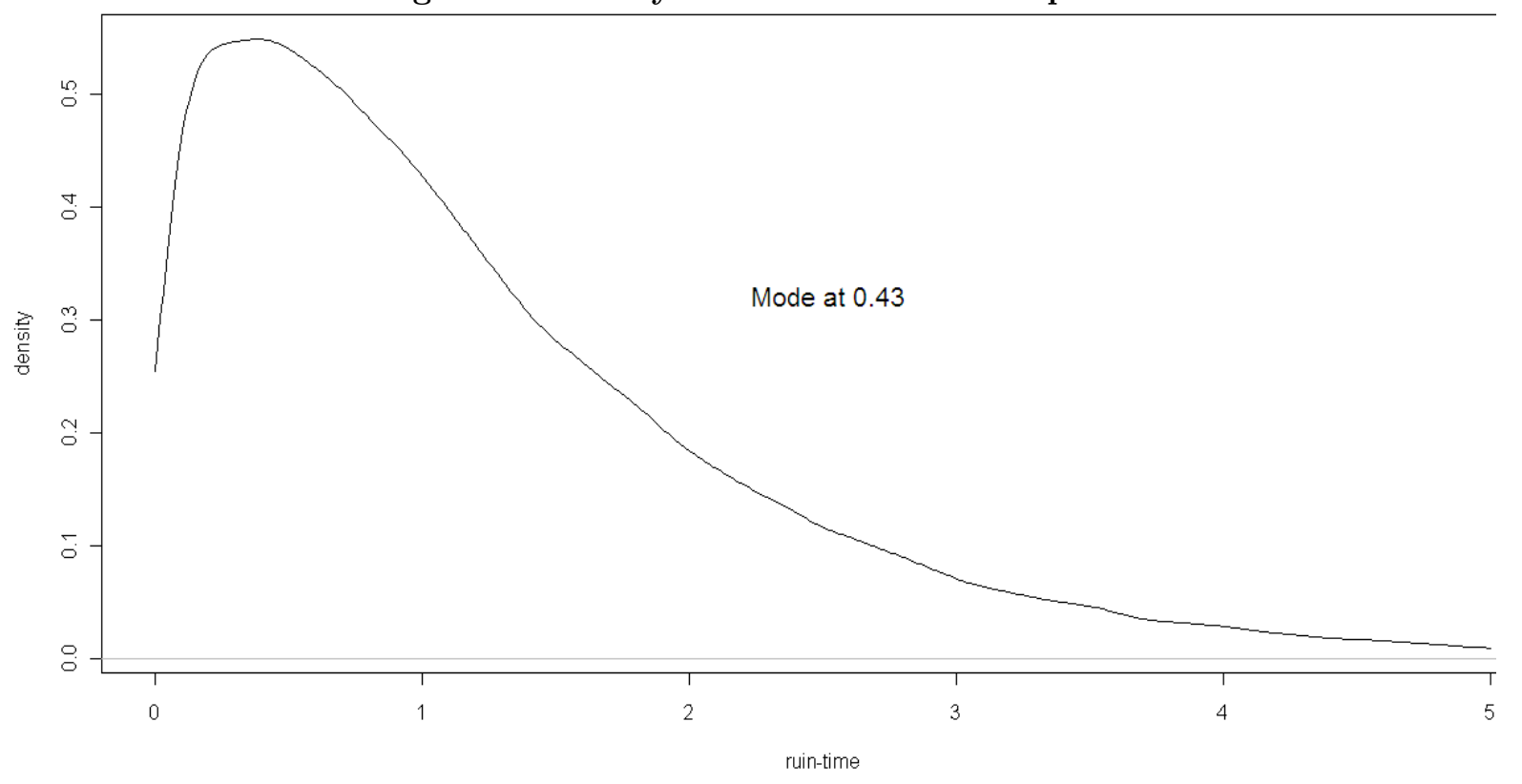


Example 3: (Discrete logarithm) To get a picture when the claim amounts are discrete, we now consider a discrete logarithm distribution as a model for severity of claims. The density function is of the form:

$$
f(i)=\frac{-\kappa^{i}}{i \times \ln (1-\kappa)}, \quad i=1,2, \ldots \text { for parameter choice of } \kappa=0.95 .
$$

While the possible values is unbounded above, in the computation we truncate it at 90 , for the given parameter choice. The mean of the distribution is

$$
\frac{-\kappa}{(1-\kappa) \times \ln (1-\kappa)}
$$

The inter-arrival of claims are again assumed to follow an exponential distribution with parameter $\lambda=30$. The risk process is generated for an initial capital of $u_{0}=50$ and a linear premium function of $p_{t}=25 t$, leading to a violation of NPC with $\theta \approx-0.8686$. Ruin time turns out to be finitely supported with a mode of 0.26 with the (kernel) density being shown in Figure 3.

Figure 3: Density of Ruin time in Example 3

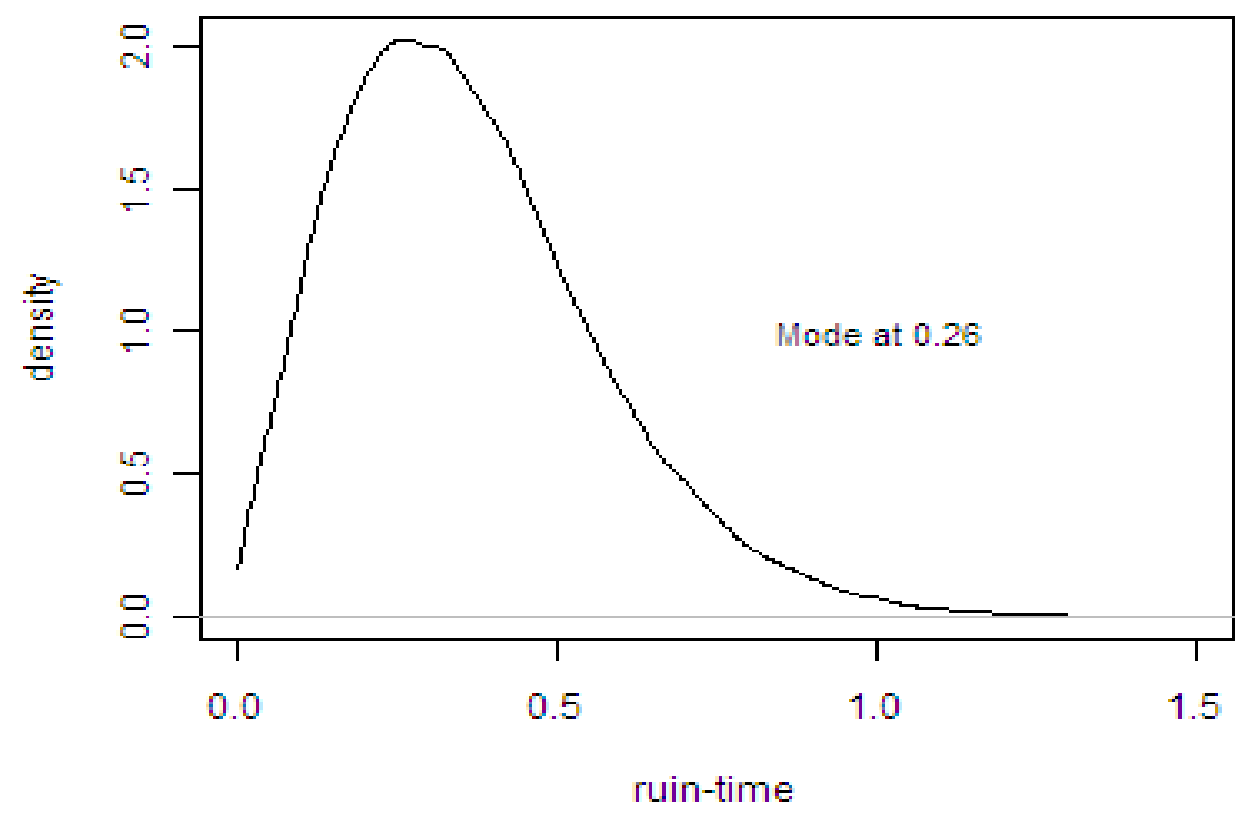

\subsubsection{How alarm time varies with its various parameters}

For Example 1, the alarm times, respectively for $\beta=0.025$ and 0.25 , are reported here in Tables 1.1 and 1.2, for various combination of $d$ and $\alpha$. Additional tables for the in-between $\beta$ values are reported in Appendix Tables A.1.1 to A.1.8. 
Table 1.1: Alarm times with $\beta=0.025$, for different $d$ and $\alpha$ Example 1: Exponential Claim Severity

\begin{tabular}{|c|ccccccccccc|}
\hline $\mathrm{d}$ & & & & & \multicolumn{1}{c|}{$\alpha$} \\
& $\mathbf{0 . 2 5}$ & $\mathbf{0 . 2 7 5}$ & $\mathbf{0 . 3}$ & $\mathbf{0 . 3 2 5}$ & $\mathbf{0 . 3 5}$ & $\mathbf{0 . 3 7 5}$ & $\mathbf{0 . 4}$ & $\mathbf{0 . 4 2 5}$ & $\mathbf{0 . 4 5}$ & $\mathbf{0 . 4 7 5}$ & $\mathbf{0 . 5}$ \\
\hline 0.75 & NA & NA & NA & NA & NA & NA & NA & NA & NA & NA & NA \\
0.8 & NA & NA & NA & NA & NA & NA & NA & NA & NA & NA & 0.12 \\
0.85 & NA & NA & NA & NA & NA & NA & NA & NA & NA & 0.11 & 0.06 \\
0.9 & NA & NA & NA & NA & NA & NA & NA & NA & 0.11 & 0.05 & 0 \\
0.95 & NA & NA & NA & NA & NA & NA & NA & 0.1 & 0.05 & 0 & 0 \\
1 & NA & NA & NA & NA & NA & NA & 0.11 & 0.05 & 0 & 0 & 0 \\
1.05 & NA & NA & NA & NA & NA & 0.11 & 0.05 & 0 & 0 & 0 & 0 \\
1.1 & NA & NA & NA & NA & 0.12 & 0.06 & 0 & 0 & 0 & 0 & 0 \\
1.15 & NA & NA & NA & 0.14 & 0.06 & 0 & 0 & 0 & 0 & 0 & 0 \\
\hline
\end{tabular}

Table 1.2: Alarm times with $\beta=0.25$, for different $d$ and $\alpha$ Example 1: Exponential Claim Severity

\begin{tabular}{|c|ccccccccccc|}
\hline $\mathrm{d}$ & & & & \multicolumn{1}{c}{$\alpha$} & & & & \\
& $\mathbf{0 . 2 5}$ & $\mathbf{0 . 2 7 5}$ & $\mathbf{0 . 3}$ & $\mathbf{0 . 3 2 5}$ & $\mathbf{0 . 3 5}$ & $\mathbf{0 . 3 7 5}$ & $\mathbf{0 . 4}$ & $\mathbf{0 . 4 2 5}$ & $\mathbf{0 . 4 5}$ & $\mathbf{0 . 4 7 5}$ & $\mathbf{0 . 5}$ \\
\hline 0.60 & NA & NA & NA & NA & NA & NA & NA & NA & NA & NA & NA \\
0.65 & NA & NA & NA & NA & NA & NA & NA & NA & NA & NA & 0.44 \\
0.7 & NA & NA & NA & NA & NA & NA & NA & NA & NA & 0.42 & 0.29 \\
0.75 & NA & NA & NA & NA & NA & NA & NA & NA & 0.4 & 0.27 & 0.19 \\
0.8 & NA & NA & NA & NA & NA & NA & NA & 0.39 & 0.27 & 0.18 & 0.12 \\
0.85 & NA & NA & NA & NA & NA & NA & 0.39 & 0.27 & 0.18 & 0.11 & 0.06 \\
0.9 & NA & NA & NA & NA & NA & 0.42 & 0.27 & 0.18 & 0.11 & 0.05 & 0 \\
0.95 & NA & NA & NA & NA & 0.45 & 0.27 & 0.18 & 0.1 & 0.05 & 0 & 0 \\
1 & NA & NA & NA & 0.49 & 0.29 & 0.18 & 0.11 & 0.05 & 0 & 0 & 0 \\
1.05 & NA & NA & NA & 0.33 & 0.2 & 0.11 & 0.05 & 0 & 0 & 0 & 0 \\
1.1 & NA & NA & 0.37 & 0.22 & 0.12 & 0.06 & 0 & 0 & 0 & 0 & 0 \\
1.15 & NA & 0.46 & 0.26 & 0.14 & 0.06 & 0 & 0 & 0 & 0 & 0 & 0 \\
\hline
\end{tabular}

For Example 2 (Pareto case), the alarm times, respectively for $\beta=0.025,0.05,0.225$ and 0.25 , are reported here in Tables 2.1 and 2.2 below, for various combination of $d$ and $\alpha$. Additional tables for the in-between $\beta$ values are reported in Appendix Tables A.2.1 to A.2.3.

Table 2.1: Alarm times with $\beta=0.025$ and 0.05 , for different $d$ and $\alpha$ Example 2: Pareto Claim Severity

\begin{tabular}{|c|c|c|c|c|c|c|c|c|c|}
\hline & \multicolumn{4}{|c|}{$\beta=0.025$} & \multirow[b]{3}{*}{ d } & \multicolumn{4}{|c|}{$\beta=0.05$} \\
\hline \multirow[t]{2}{*}{ d } & \multicolumn{4}{|c|}{$\alpha$} & & \multicolumn{4}{|c|}{$\alpha$} \\
\hline & 0.4 & 0.425 & 0.45 & 0.475 & & 0.4 & 0.425 & 0.45 & 0.475 \\
\hline 0.95 & NA & NA & $\mathrm{NA}$ & NA & 0.95 & NA & NA & NA & NA \\
\hline 1 & NA & NA & NA & NA & 1 & NA & NA & NA & 0.07 \\
\hline 1.05 & NA & NA & NA & 0 & 1.05 & NA & NA & 0.09 & 0 \\
\hline 1.1 & NA & NA & 0 & 0 & 1.1 & NA & NA & 0 & 0 \\
\hline 1.15 & NA & 0.01 & 0 & 0 & 1.15 & NA & 0.01 & 0 & 0 \\
\hline
\end{tabular}

Table 2.2: Alarm times with $\beta=0.225$ and 0.25 , for different $d$ and $\alpha$ Example 2: Pareto Claim Severity

\begin{tabular}{|c|c|c|c|c|c|c|c|c|c|c|c|c|}
\hline & \multicolumn{5}{|c|}{$\beta=0.225$} & & \multicolumn{6}{|c|}{$\beta=0.25$} \\
\hline \multirow[t]{2}{*}{$\mathrm{d}$} & \multicolumn{5}{|c|}{$\alpha$} & & \multicolumn{6}{|c|}{$\alpha$} \\
\hline & 0.375 & 0.4 & 0.425 & 0.45 & 0.475 & $\mathrm{~d}$ & 0.35 & 0.375 & 0.4 & 0.425 & 0.45 & 0.475 \\
\hline 0.85 & NA & NA & NA & NA & NA & 0.85 & NA & NA & NA & NA & NA & NA \\
\hline 0.9 & NA & NA & NA & NA & 0.39 & 0.9 & NA & NA & NA & NA & NA & 0.39 \\
\hline 0.95 & NA & NA & NA & NA & 0.21 & 0.95 & NA & NA & NA & NA & 0.43 & 0.21 \\
\hline 1 & NA & NA & NA & 0.24 & 0.07 & 1 & NA & NA & NA & NA & 0.24 & 0.07 \\
\hline 1.15 & NA & 0.18 & 0.01 & 0 & 0 & 1.15 & NA & 0.45 & 0.18 & 0.01 & 0 & 0 \\
\hline
\end{tabular}


Table 2.2: Alarm times with $\beta=0.25$, for different $d$ and $\alpha$ Example 2: Pareto Claim Severity

\begin{tabular}{|c|cccccccccc|}
\hline $\mathrm{d}$ & & & \multicolumn{10}{c|}{$\alpha$} \\
& $\mathbf{0 . 2 5}$ & $\mathbf{0 . 2 7 5}$ & $\mathbf{0 . 3}$ & $\mathbf{0 . 3 2 5}$ & $\mathbf{0 . 3 5}$ & $\mathbf{0 . 3 7 5}$ & $\mathbf{0 . 4}$ & $\mathbf{0 . 4 2 5}$ & $\mathbf{0 . 4 5}$ & $\mathbf{0 . 4 7 5}$ \\
\hline 0.8 & NA & NA & NA & NA & NA & NA & NA & NA & NA & NA \\
0.85 & NA & NA & NA & NA & NA & NA & NA & NA & NA & NA \\
0.9 & NA & NA & NA & NA & NA & NA & NA & NA & NA & 0.39 \\
0.95 & NA & NA & NA & NA & NA & NA & NA & NA & 0.43 & 0.21 \\
1 & NA & NA & NA & NA & NA & NA & NA & NA & 0.24 & 0.07 \\
1.05 & NA & NA & NA & NA & NA & NA & NA & 0.28 & 0.09 & 0 \\
1.1 & NA & NA & NA & NA & NA & NA & 0.35 & 0.13 & 0 & 0 \\
1.15 & NA & NA & NA & NA & NA & 0.45 & 0.18 & 0.01 & 0 & 0 \\
\hline
\end{tabular}

For Example 3 (discrete logarithm case), the alarm times, respectively for $\beta=0.025$ and 0.25 , are reported here in Tables 3.1 and Tables 3.2, for various combination of $d$ and $\alpha$. Additional tables for the in-between $\beta$ values are reported in Appendix Tables A.3.1 to A.3.8.

Table 3.1: Alarm times with $\beta=0.025$, for different $d$ and $\alpha$ Example 3: Discrete Logarithm Claim Severity

\begin{tabular}{|c|c|c|c|c|c|c|c|c|c|c|}
\hline \multirow[t]{2}{*}{ d } & \multicolumn{10}{|c|}{$\alpha$} \\
\hline & 0.25 & 0.275 & 0.3 & 0.325 & 0.35 & 0.375 & 0.4 & 0.425 & 0.45 & 0.475 \\
\hline 0.29 & NA & NA & NA & NA & NA & NA & NA & NA & NA & NA \\
\hline 0.31 & NA & NA & NA & NA & NA & NA & NA & NA & NA & 0.05 \\
\hline 0.33 & NA & NA & NA & NA & NA & NA & NA & NA & 0.04 & 0.03 \\
\hline 0.35 & NA & NA & NA & NA & NA & NA & 0.05 & 0.03 & 0.02 & 0 \\
\hline 0.37 & NA & NA & NA & NA & NA & 0.04 & 0.03 & 0.01 & 0 & 0 \\
\hline 0.39 & NA & NA & NA & 0.05 & 0.04 & 0.02 & 0.01 & 0 & 0 & 0 \\
\hline 0.41 & NA & NA & 0.05 & 0.03 & 0.02 & 0 & 0 & 0 & 0 & 0 \\
\hline 0.43 & NA & 0.05 & 0.03 & 0.01 & 0 & 0 & 0 & 0 & 0 & 0 \\
\hline 0.45 & 0.04 & 0.02 & 0.01 & 0 & 0 & 0 & 0 & 0 & 0 & 0 \\
\hline 0.47 & 0.03 & 0.01 & 0 & 0 & 0 & 0 & 0 & 0 & 0 & 0 \\
\hline 0.49 & 0 & 0 & 0 & 0 & 0 & 0 & 0 & 0 & 0 & 0 \\
\hline 0.51 & 0 & 0 & 0 & 0 & 0 & 0 & 0 & 0 & 0 & 0 \\
\hline 0.53 & 0 & 0 & 0 & 0 & 0 & 0 & 0 & 0 & 0 & 0 \\
\hline
\end{tabular}

Table 3.2: Alarm times with $\beta=0.25$, for different $d$ and $\alpha$ Example 3: Discrete Logarithm Claim Severity

\begin{tabular}{|c|c|c|c|c|c|c|c|c|c|c|}
\hline \multirow[t]{2}{*}{$\mathrm{d}$} & \multicolumn{10}{|c|}{$\alpha$} \\
\hline & 0.25 & 0.275 & 0.3 & 0.325 & 0.35 & 0.375 & 0.4 & 0.425 & 0.45 & 0.475 \\
\hline 0.25 & NA & NA & NA & NA & NA & NA & 0.19 & 0.17 & 0.15 & 0.13 \\
\hline 0.27 & NA & NA & NA & NA & 0.21 & 0.18 & 0.16 & 0.14 & 0.12 & 0.1 \\
\hline 0.29 & NA & NA & NA & 0.2 & 0.17 & 0.15 & 0.13 & 0.11 & 0.09 & 0.07 \\
\hline 0.31 & NA & NA & 0.19 & 0.16 & 0.14 & 0.12 & 0.1 & 0.08 & 0.06 & 0.05 \\
\hline 0.33 & 0.21 & 0.18 & 0.15 & 0.13 & 0.11 & 0.09 & 0.07 & 0.06 & 0.04 & 0.03 \\
\hline 0.35 & 0.18 & 0.15 & 0.13 & 0.1 & 0.08 & 0.07 & 0.05 & 0.03 & 0.02 & 0 \\
\hline 0.37 & 0.15 & 0.12 & 0.1 & 0.08 & 0.06 & 0.04 & 0.03 & 0.01 & 0 & 0 \\
\hline 0.39 & 0.12 & 0.09 & 0.07 & 0.05 & 0.04 & 0.02 & 0.01 & 0 & 0 & 0 \\
\hline 0.41 & 0.09 & 0.07 & 0.05 & 0.03 & 0.02 & 0 & 0 & 0 & 0 & 0 \\
\hline 0.43 & 0.07 & 0.05 & 0.03 & 0.01 & 0 & 0 & 0 & 0 & 0 & 0 \\
\hline 0.45 & 0.04 & 0.02 & 0.01 & 0 & 0 & 0 & 0 & 0 & 0 & 0 \\
\hline 0.47 & 0.03 & 0.01 & 0 & 0 & 0 & 0 & 0 & 0 & 0 & 0 \\
\hline 0.49 & 0 & 0 & 0 & 0 & 0 & 0 & 0 & 0 & 0 & 0 \\
\hline
\end{tabular}

Observations. The observations from this simulation exercise reconfirm the intuitive dependency of alarm times on $\alpha, \beta$ and $d$, viz.

- For fixed $\alpha$ and $\beta$, the alarm time decreases with increase in $d$; for too small a choice of $d$, alarm never happens, and for too large a $d$, the alarm sounds instantaneously (alarm time 
$=0)$. This is intuitively justified as the ruin probability increases with the future time horizon $(d)$.

- For fixed $d$ and $\beta$, the alarm time decreases with increase in $\alpha$. This is also intuitively justifiable as an increase in $\alpha$ amounts to be less restrictive i.e. more proactive in taking precautionary measures.

- The impact of $\beta$ on the alarm time is interesting and perhaps less intuitive. It appears that, if the alarm happens then actually the timing of the alarm does not depend on $\beta$. Of course it is possible that for some small $\beta$, alarm does not sound ever while it does for large $\beta$; e.g. compare the alarm times for $d=1.15, \alpha=0.275$ from Table 1.1 and Table 1.2.

\subsubsection{How alarm time depends on Initial Capital}

Next we investigate how the alarm times change with initial capital. It is intuitively obvious that the alarm time would increase with an increase in the initial capital. To observe the pattern in a specific instance, we revert to the setting in Example 2, except the initial capital is varied from 0 to 120 and we observe the alarm times with $\beta=0.225, \alpha=0.45$ and $d=1.0$. The alarm times are noted in the following table:

Table 4: First Alarm time for different initial capitals in Example 2

\begin{tabular}{|c|c|c|c|c|c|c|c|c|c|c|c|c|c|}
\hline $\mathrm{u}$ & 0 & 5 & 10 & 15 & 20 & 25 & 30 & 35 & 40 & 45 & 50 & 55 & \\
\hline alarm & 0 & 0 & 0 & 0 & 0 & 0 & 0 & 0 & 0 & 0.05 & 0.26 & 0.5 & \\
\hline $\mathrm{u}$ & 60 & 65 & 70 & 75 & 80 & 85 & 90 & 95 & 100 & 105 & 110 & 115 & 120 \\
\hline alarm & 0.79 & 1.06 & 1.41 & 1.69 & 2.13 & 2.45 & 2.92 & 3.62 & 3.86 & 4.44 & 4.76 & 6.25 & 7.01 \\
\hline
\end{tabular}

Figure 4 provides a visual representation of this. As we can see the alarm sounds instantaneously unless the initial capital is at least 45 (possibly marginally less); for higher initial capital, alarm time is delayed almost linearly till the initial capital is 110. For higher initial capital, the alarm time grows at a much higher rate and eventually alarm will not sound; indeed, there would be no ruin.

Figure 4: First alarm time for various initial capital amounts in Example 2

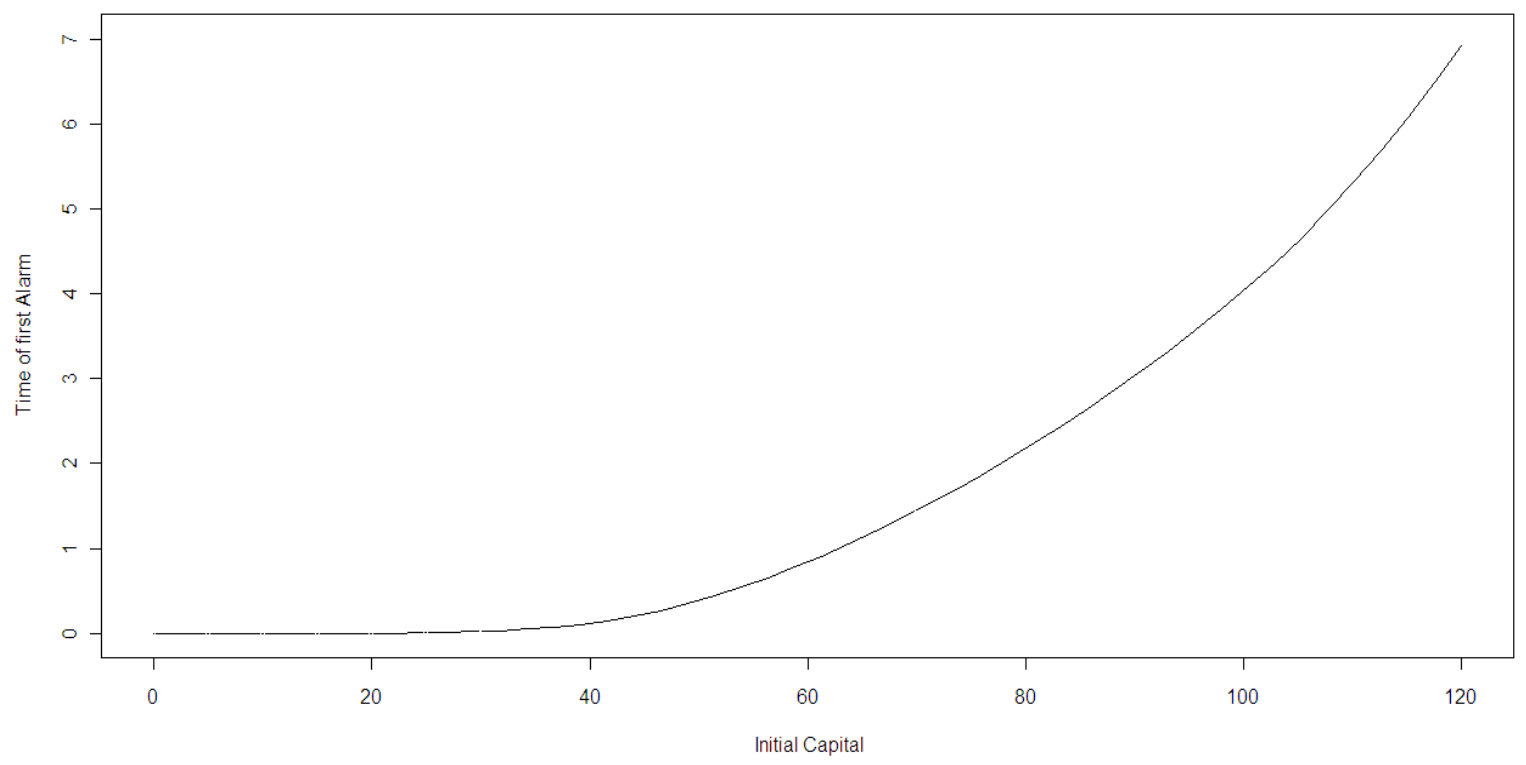


It would be interesting to see how the infinite time (as well as possibly finite time, at different time points) ruin probabilities change with various amounts put as initial capital. To see this, we compare the entire ruin-time distributions for the various choices of the initial capitals; this is being shown in the following set of diagrams in Figure 5.

Figure 5: Density of Ruin-time with various Initial Capitals
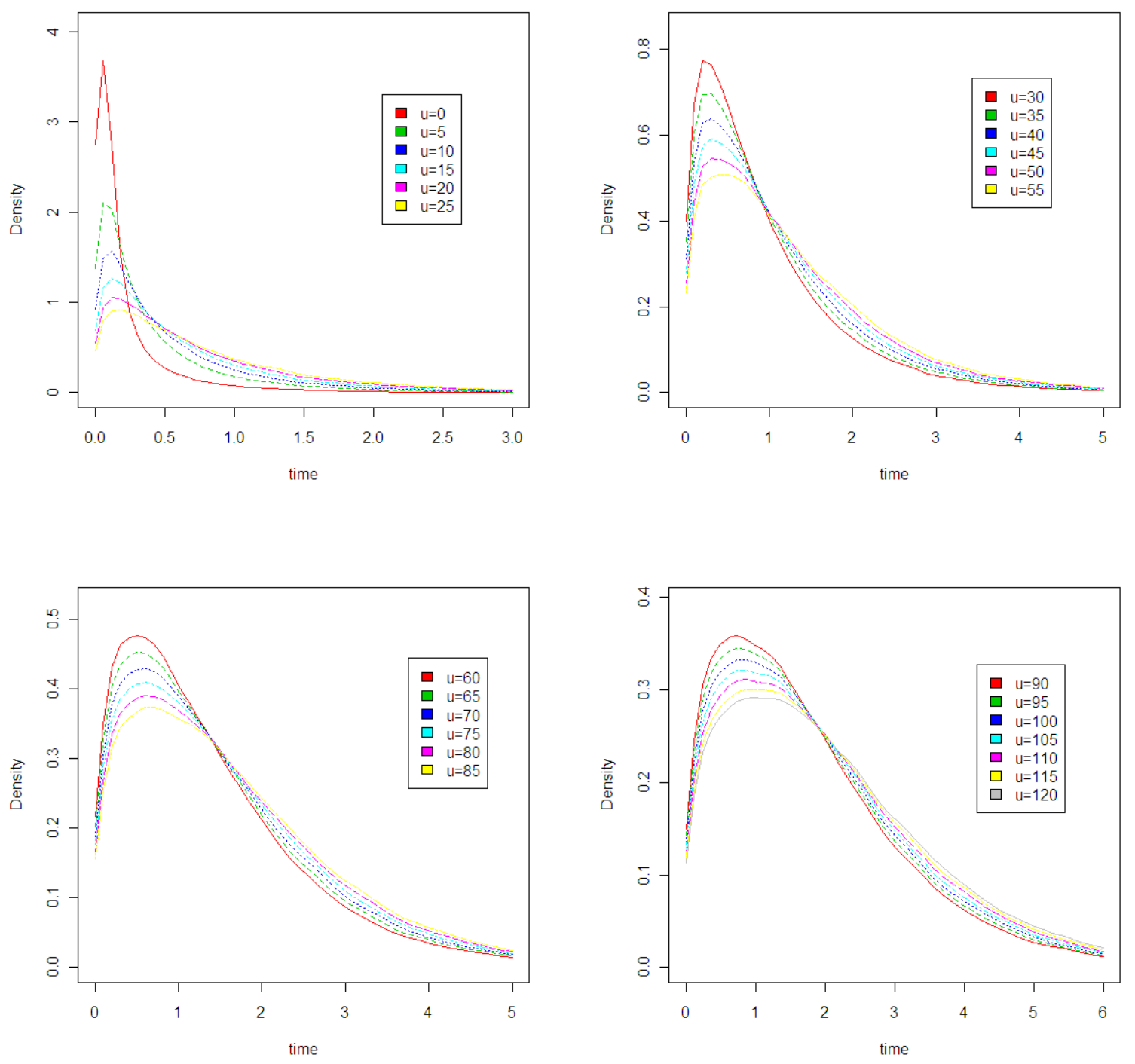

It may be more interesting to compare the probabilities of ruin before a given time $(t)$ directly; this is done for a selection of initial capitals in Figure 6. 
Figure 6: Finite probability of Ruin for various initial capital amounts

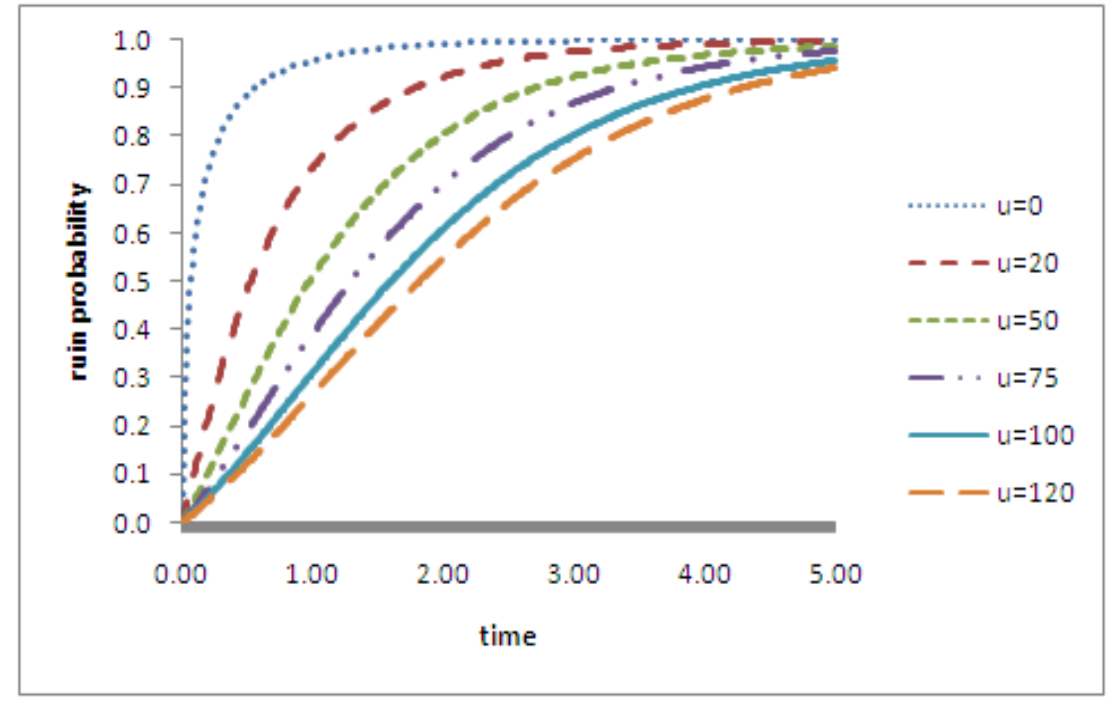

\subsection{Multiple Alarms or An Alarm System with Capital Added at each Alarm Time}

Suppose we have at time 0 a capital $u_{0}$. To prevent the system from ruin, we propose to add a capital $u_{1}$ at time $A$. We generalize this procedure and define a system of alarm times $A_{i}, i \geq 1$, adding a capital $u_{i}$ at every alarm time $A_{i}$. Note that the risk process $V(\cdot)$ gets then modified at each $A_{i}, i \geq 1$.

Let us define the event $B_{i}$ as

$$
B_{i}:=\left(\text { no ruin occurs up to alarm time } A_{i}\right)=\bigcap_{n=1}^{i}\left(T\left(A_{n-1}, \sum_{m=0}^{n-1} u_{m}\right)>A_{n}\right) \text {. }
$$

So, setting $A_{0}:=0$, we define for $i \geq 1$

$$
\begin{aligned}
& A_{i+1}=\inf \left\{s>A_{i}: P\left[T\left(A_{i}, \sum_{n=0}^{i} u_{n}\right) \leq s+d \mid\left(T\left(A_{i}, \sum_{n=0}^{i} u_{n}\right)>s\right) \cap B_{i}\right] \geq 1-\alpha\right. \\
& \left.\quad \text { and } P\left[T\left(A_{i}, \sum_{n=0}^{i} u_{n}\right)>s \mid B_{i}\right] \geq 1-\beta\right\} \\
& =\inf \left\{s>A_{i}: P\left[s<T\left(A_{i}, \sum_{n=0}^{i} u_{n}\right) \leq s+d \mid B_{i}\right] \geq(1-\alpha) P\left[T\left(A_{i}, \sum_{n=0}^{i} u_{n}\right)>s \mid B_{i}\right]\right. \\
& \text { and } \left.P\left[T\left(A_{i}, \sum_{n=0}^{i} u_{n}\right)>s \mid B_{i}\right] \geq 1-\beta\right\} \\
& =\inf \left\{s>A_{i}: \psi_{A_{i}}\left(\sum_{n=0}^{i} u_{n}, s+d \mid B_{i}\right)-\psi_{A_{i}}\left(\sum_{n=0}^{i} u_{n}, s \mid B_{i}\right) \geq\right. \\
& \left.(1-\alpha) \bar{\psi}_{A_{i}}\left(\sum_{n=0}^{i} u_{n}, s \mid B_{i}\right) \quad \text { and } \bar{\psi}_{A_{i}}\left(\sum_{n=0}^{i} u_{n}, s \mid B_{i}\right) \geq 1-\beta\right\} .
\end{aligned}
$$


In particular, this definition implies that

$$
\psi_{A_{i}}\left(\sum_{n=0}^{i} u_{n}, s+d \mid B_{i}\right)-\psi_{A_{i}}\left(\sum_{n=0}^{i} u_{n}, s \mid B_{i}\right) \geq(1-\alpha)(1-\beta) .
$$

Remark: For the sake of simplicity, we keep here the same values for $\alpha, \beta$ and $d$ in the definition of successive alarm times; however, to generalize, one may consider a sequence of values $\left(\alpha_{i}\right)_{i \geq 1}$, $\left(\beta_{i}\right)_{i \geq 1}$ and $\left(d_{i}\right)_{i \geq 1}$ instead. The capital $u_{i}$ added at each alarm time $A_{i}$ is on purpose given; it corresponds in this way to the (available) amount that the company chooses to put at this time. The advantage is that it gives more flexibility to the company, but it also implies that the smallest the added capital are, the closest the alarm times may become. The same procedure is then repeated from any alarm time $A_{i}$ with the associated capital $\sum_{n=0}^{i} u_{n}$. An alternative way would be to optimize the amount $u_{i}$ to be put at an alarm time $A_{i}$, such that the chance of ruin $P\left[T\left(A_{i}, \sum_{n=0}^{i} u_{n}\right) \leq A_{i}+d\right]$ is very small. In this case, the procedure would start again at $A_{i}+d$ instead of at $A_{i}$.

Let us now report the computational results in terms of timings of multiple alarms, in the context of Example 1, Example 2 and Example 3.

Revisit Example 1. We consider the similar risk process, i.e. claim severity has an exponential $(\rho=0.5)$ distribution with claim process being a Poisson process $(\lambda=20)$, premium function being $p_{t}=25 t$; only the initial capital $u$ being changed to 17.5. As for the parameters in the definition of alarm, we take $\beta=0.225, \alpha=0.45$ and $d=1.0$. A fixed percentage of the initial capital is added to the capital at the sound of each alarm; in the following table, we report the pattern of the first few alarm times, for the different choice of this percentage of the initial capital, that would be added to the system:

Table 5: (Example 1) Timings of the first 10 alarms as a function of amounts added at each alarm

\begin{tabular}{|c|c|c|c|c|c|c|c|c|c|c|c|}
\hline $\begin{array}{c}\% \text { of } \mathrm{u} \text { added } \\
\text { at each alarm }\end{array}$ & alarm 1 & alarm 2 & alarm 3 & alarm 4 & alarm 5 & alarm 6 & alarm 7 & alarm 8 & alarm 9 & alarm 10 \\
\hline $1 \%$ & 0.18 & 0.20 & 0.22 & 0.23 & 0.25 & 0.27 & 0.28 & 0.30 & 0.32 & 0.34 \\
$2.5 \%$ & 0.18 & 0.22 & 0.26 & 0.31 & 0.35 & 0.39 & 0.45 & 0.50 & 0.56 & 0.61 \\
$5 \%$ & 0.18 & 0.26 & 0.34 & 0.44 & 0.53 & 0.64 & 0.76 & 0.90 & 1.04 & 1.18 \\
$10 \%$ & 0.18 & 0.35 & 0.54 & 0.76 & 1.03 & 1.32 & 1.66 & 2.01 & 2.37 & NA \\
$20 \%$ & 0.18 & 0.53 & NA & NA & NA & NA & NA & NA \\
$30 \%$ & 0.18 & 0.72 & NA & NA & NA & NA & NA & NA & NA & NA \\
\hline
\end{tabular}

Revisit Example 2. We now revisit the same risk process as in Example 2 and consider alarms with $\beta=0.225, \alpha=0.45$ and $d=1.0$. With $10 \%$ of the initial capital $u=50$, being added to the system at each alarm, we get exactly 4 alarms occurring respectively at $t=0.29,0.58,0.91$, and 1.28 .

Revisit Example 3. With the setup of Example 3, claim severity having discrete logarithmic distribution with $\kappa=0.95$, we take $\beta=0.05, \alpha=0.4$ and $d=0.35$ as the parameters in the definition of alarm. Table $6 \mathrm{~A}$ is with an initial capital of $u=50$, Table $6 \mathrm{~B}$ is with $u=45$, while Table $6 \mathrm{C}$ is with $u=55$; premium function being $p_{t}=25 t$ in either setup. 
Table 6A: (Example 3) First 3 alarm times as a function of amounts added at each alarm

\begin{tabular}{||c|c|c|c||}
\hline \multicolumn{1}{|c|}{$\%$ of u added } & \multicolumn{3}{|c|}{ alarm times } \\
at each alarm & alarm 1 & alarm 2 & alarm 3 \\
\hline $2 \%$ & 0.05 & 0.06 & 0.07 \\
$4 \%$ & 0.05 & 0.06 & 0.08 \\
$6 \%$ & 0.05 & 0.07 & 0.09 \\
$8 \%$ & 0.05 & 0.08 & 0.11 \\
$10 \%$ & 0.05 & 0.09 & 0.13 \\
\hline
\end{tabular}

Table 6B: (Example 3) First 5 alarm times as a function of amounts added at each alarm

\begin{tabular}{|c|c|c|c|c|c||}
\hline $\begin{array}{c}\text { \% of u added } \\
\text { at each alarm }\end{array}$ & \multicolumn{6}{|c|}{ alarm 1 } & alarm 2 & alarm 3 & alarm 4 & alarm 5 \\
\hline $10 \%$ & 0.01 & 0.04 & 0.08 & 0.11 & 0.15 \\
$20 \%$ & 0.01 & 0.08 & 0.15 & NA & NA \\
$30 \%$ & 0.01 & NA & NA & NA & NA \\
$40 \%$ & 0.01 & NA & NA & NA & NA \\
$50 \%$ & 0.01 & NA & NA & NA & NA \\
\hline
\end{tabular}

Table 6C: (Example 3) First 5 alarm times as a function of amounts added at each alarm

\begin{tabular}{|c|c|c|c|c|c||}
\hline \% of u added & \multicolumn{6}{|c|}{ alarm times } \\
at each alarm & alarm 1 & alarm 2 & alarm 3 & alarm 4 & alarm 5 \\
\hline $\mathbf{2 . 5 \%}$ & 0.08 & 0.09 & 0.1 & 0.12 & 0.13 \\
$\mathbf{5 \%}$ & 0.08 & 0.11 & 0.13 & 0.15 & 0.18 \\
$\mathbf{7 . 5 \%}$ & 0.08 & 0.12 & 0.15 & 0.19 & 0.22 \\
$\mathbf{1 0 \%}$ & 0.08 & 0.13 & 0.17 & 0.22 & 0.27 \\
$\mathbf{1 2 . 5 \%}$ & 0.08 & 0.14 & 0.19 & NA & NA \\
$\mathbf{1 5 \%}$ & 0.08 & 0.15 & NA & NA & NA \\
$\mathbf{1 7 . 5 \%}$ & 0.08 & 0.16 & NA & NA & NA \\
$\mathbf{2 0 \%}$ & 0.08 & NA & NA & NA & NA \\
\hline
\end{tabular}

\section{Effectiveness of Alarm System}

We consider the alarm system as an alternate strategy for having to put up an excessive initial capital to avoid ruin. This alternative strategy calls for starting with a comparatively modest initial capital, while additional amounts are to be added to the capital whenever the suitably designed alarms go off. To draw a fair comparison, we consider the total capital to be equivalent under the two alternatives. Mathematically, as well as intuitively, it is clear that the probability of ruin would be lower when one puts the total amount at the very beginning; however we look for devising a suitable alarm system where the difference in ruin probability is nominal, especially when considering the time value of money. More precisely, as described in the alarm system, whenever 'alarm' sounds, a designed amount of capital may be added to the accumulation function to prevent from ruin, providing also a new risk process for which we would define the new alarm time. Thus, in essence, we are proposing a piecewise linear accumulation function with discontinuities at the time point of alarms by upward parallel shift of the function.

\subsection{Comparison with one alarm time model}

First consider the case of two models, one named $M^{A}$ when considering an alarm system with one known (deterministic or random) alarm time $A$ only and adding to the initial amount $u_{0}$ a certain amount $u_{1}$ at $A$, and the other named $M_{r}$ when we do not take into account any alarm time but 
put an initial discounted amount $u_{0}+e^{-r A} u_{1}$, where $r$ denotes a continuously compound interest rate. We want to compare the probability of ruin (in finite or infinite time) of those two models. We will add the symbols $M_{r}$ and $M^{A}$ to our previous notations whenever it will be necessary to precise under which of those two models we are working, $M_{0}$ corresponding when no consideration of time value for money is made. Let us denote the ruin times under two set up by $T^{\left(M_{r}\right)}$ and $T^{M^{A}}$.

- Under the NPC, i.e. $\Theta>0$, we will compare the finite time and infinite time ruin probabilities.

- If the NPC is violated, we have

$$
P[T(u)=\infty]=0, \quad \forall u,
$$

which makes the comparison of infinite horizon survival probabilities worthless. So we focus on comparing the ruin probabilities over finite time only.

In the following, we compare the two models $M_{r}$ and $M^{A}$ by first giving an exact analytical expression of the difference $\Delta(t)$ in the finite time ruin probabilities, i.e.

$$
\Delta(t):=P\left[T^{M^{A}} \leq t\right]-P\left[T^{M_{r}} \leq t\right], \text { for } t>0,
$$

next upper and lower bounds of this difference.

The results will be extended as $t \rightarrow \infty$ to study the difference $\Delta$ in the ultimate ruin probabilities.

As we would observe, the finite horizon ruin probability comparison via $\Delta(t)$ between the two systems is significant, unlike the infinite horizon comparison, irrespective of whether the NPC is satisfied or not. Intuitively, it is reasonable to expect that the alarm system should hold the edge if the rate of interest is moderately high; it would also depend on how late the alarm is put in place and also on the time horizon considered, i.e. the specific values of $A$ and $t$. The goal of this section is to validate such an assertion and possibly obtain objective results in that direction, with specific focus on alarm defined in (12).

a) Finite time Ruin Probabilities In carrying out the comparison, we find that the difference critically depends on whether the time considered $t$ is beyond the alarm time $A$ or not; accordingly segregate the two cases.

- If $t \leq A$, then $\left(T^{M^{A}} \leq t\right)=\left(T\left(u_{0}\right) \leq t\right)$ and so

$$
\Delta(t)=\psi\left(u_{0}, t\right)-\psi\left(u_{0}+e^{-r A} u_{1}, t\right) .
$$

Since $\psi(u, t)$ is a non-decreasing function of the time $t$ and a non-increasing function of the capital $u$, we obtain

$$
0 \leq \Delta(t) \leq \psi\left(u_{0}, A\right)-\psi\left(u_{0}+e^{-r A} u_{1}, t\right) \leq \psi\left(u_{0}, A\right)-\psi\left(u_{0}+e^{-r t} u_{1}, t\right) .
$$

In this case, model $M_{r}$ has an edge over the model $M^{A}$, as is intuitively obvious, with the advantage reducing with increase in $A$.

With the alarm time $A$ defined as in (12), (18) becomes

$$
0 \leq \Delta(t) \leq \beta-\psi\left(u_{0}+e^{-r A} u_{1}, t\right) \leq \beta-\psi\left(u_{0}+e^{-r t} u_{1}, t\right) \leq \beta .
$$

The upper bound being $\beta$, it can be small enough. 
- Suppose $t>A$. First observe that we can write, in the same way as for (5), that for any $t>a$ (including $t=\infty$ ) and for two amounts $u<v$,

$$
\begin{aligned}
P[T(u)>a, T(a, v) \leq t] & =P\left[\sup _{0<s \leq a} R_{s} \leq u, \sup _{a<s \leq t} R_{s}>v\right] \\
& =\int_{-\infty}^{u} P\left[\sup _{0<s \leq a} R_{s} \leq u, \sup _{a<s \leq t} R_{s}-R_{a}>v-x \mid R_{a}=x\right] d F_{R_{a}}(x) \\
& =\int_{-\infty}^{u} \psi(v-x, t-a) \bar{\psi}\left(u, a \mid R_{a}=x\right) d F_{R_{a}}(x)
\end{aligned}
$$

and $P[T(u) \leq a, T(v)>t]$

$$
\begin{aligned}
& =P\left[\sup _{0<s \leq a} R_{s}>u, \sup _{s \leq t} R_{s} \leq v\right] \\
& =\int_{-\infty}^{v} P\left[u<\sup _{0<s \leq a} R_{s} \leq v, \sup _{a<s \leq t} R_{s}-R_{a} \leq v-x \mid R_{a}=x\right] d F_{R_{a}}(x) \\
& =\int_{-\infty}^{v} \bar{\psi}(v-x, t-a)\left(\psi\left(u, a \mid R_{a}=x\right)-\psi\left(v, a \mid R_{a}=x\right)\right) d F_{R_{a}}(x) .
\end{aligned}
$$

Both expressions will be useful to obtain lower and upper bounds for $\Delta(t)$.

Now let us compute $\Delta(t)$.

$$
\begin{aligned}
\Delta(t)= & P\left[T\left(u_{0}+e^{-r A} u_{1}\right)>t\right]-P\left[T\left(u_{0}\right)>A, T\left(A, u_{0}+u_{1}\right)>t\right] \\
= & P\left[T\left(u_{0}+e^{-r A} u_{1}\right)>t\right]-P\left[T\left(u_{0}\right)>A\right]+P\left[T\left(u_{0}\right)>A, T\left(A, u_{0}+u_{1}\right) \leq t\right] \\
= & \psi\left(u_{0}, A\right)-\psi\left(u_{0}+e^{-r A} u_{1}, t\right)+ \\
& \int_{-\infty}^{u_{0}} \psi\left(u_{0}+u_{1}-x, t-A\right) \bar{\psi}\left(u_{0}, A \mid R_{A}=x\right) d F_{R_{A}}(x) \text { using }(19) \\
= & \bar{\psi}\left(u_{0}+e^{-r A} u_{1}\right)-\int_{-\infty}^{u_{0}} \bar{\psi}\left(u_{0}+u_{1}-x\right) \bar{\psi}\left(u_{0}, A \mid R_{A}=x\right) d F_{R_{A}}(x) .
\end{aligned}
$$

Alternatively,

$$
\begin{aligned}
\Delta(t)= & P\left[T\left(u_{0}+e^{-r A} u_{1}\right)>t\right]-P\left[T\left(u_{0}\right)>A, T\left(u_{0}+u_{1}\right)>t\right] \\
= & P\left[T\left(u_{0}+e^{-r A} u_{1}\right)>t\right]-P\left[T\left(u_{0}+u_{1}\right)>t\right]+P\left[T\left(u_{0}\right) \leq A, T\left(u_{0}+u_{1}\right)>t\right] \\
= & \psi\left(u_{0}+u_{1}, t\right)-\psi\left(u_{0}+e^{-r A} u_{1}, t\right)+ \\
& \int_{-\infty}^{u_{0}+u_{1}} \bar{\psi}\left(u_{0}+u_{1}-x, t-A\right)\left(\psi\left(u_{0}, A \mid R_{A}=x\right)-\psi\left(u_{0}+u_{1}, A \mid R_{A}=x\right)\right) d F_{R_{A}}(x)
\end{aligned}
$$

using (20).

Let us now obtain bounds for $\Delta(t)$. Since $\psi$ is a non-increasing function of the capital, the integral term in (22) satisfies

$$
\begin{aligned}
& \int_{-\infty}^{u_{0}} \psi\left(u_{0}+u_{1}-x, t-A\right) \bar{\psi}\left(u_{0}, A \mid R_{A}=x\right) d F_{R_{A}}(x) \\
& \quad \leq \psi\left(u_{1}, t-A\right) \int_{-\infty}^{u_{0}} \bar{\psi}\left(u_{0}, A \mid R_{A}=x\right) d F_{R_{A}}(x)=\psi\left(u_{1}, t-A\right) \bar{\psi}\left(u_{0}, A\right)
\end{aligned}
$$


whereas the one in (23) satisfies

$$
\begin{aligned}
& \int_{-\infty}^{u_{0}+u_{1}} \bar{\psi}\left(u_{0}+u_{1}-x, t-A\right)\left(\psi\left(u_{0}, A \mid R_{A}=x\right)-\psi\left(u_{0}+u_{1}, A \mid R_{A}=x\right)\right) d F_{R_{A}}(x) \\
& \quad \geq \psi(0, t-A) \int_{-\infty}^{u_{0}+u_{1}} P\left[u_{0}<\sup _{s \leq A} R_{s} \leq u_{0}+u_{1} \mid R_{A}=x\right] d F_{R_{A}}(x) \\
& \quad=\bar{\psi}(0, t-A)\left(\psi\left(u_{0}, A\right)-\psi\left(u_{0}+u_{1}, A\right)\right) .
\end{aligned}
$$

Hence, using those results leads to conclude to the following proposition, when considering the case $t>A$ (since for $t \leq A$, it is trivial).

Proposition 1 Let $r$ denote the continuously compounded interest rate. The difference $\Delta(t)$ between the finite time ruin probabilities in the two models, viz. $\left(M_{r}\right)$ (with initial capital $u_{0}+e^{-r t_{A}} u_{1}$ ) and $\left(M^{A}\right)$ (with initial capital $u_{0}$ up to the alarm time $A$, then with an extra amount of $u_{1}$ put at $A)$, can be bounded for any $t>A$ in the following way.

(i) For any deterministic alarm time A, we have

$$
\begin{aligned}
\Delta(t) & \leq \psi\left(u_{0}, A\right) \bar{\psi}\left(u_{1}, t-A\right)+\psi\left(u_{1}, t-A\right)-\psi\left(u_{0}+e^{-r A} u_{1}, t\right) \\
& \leq 1-\bar{\psi}\left(u_{0}, A\right) \bar{\psi}\left(u_{1}, t-A\right)-\psi\left(u_{0}+u_{1}, t\right)
\end{aligned}
$$

and

$$
\begin{aligned}
\Delta(t) \geq \max \left\{\psi\left(u_{0}+e^{-r A} u_{1}, t\right)-\min \left(\bar{\psi}\left(u_{0}, A\right), \bar{\psi}_{A}\left(u_{0}+u_{1}, t\right)\right) ;\right. \\
\left.\psi\left(u_{0}+u_{1}, t\right)-\psi\left(u_{0}+e^{-r A} u_{1}, t\right)+\bar{\psi}(0, t-A)\left[\psi\left(u_{0}, A\right)-\psi\left(u_{0}+u_{1}, A\right)\right]\right\} \\
\geq \psi\left(u_{0}+u_{1}, t\right)-\min \left\{\bar{\psi}\left(u_{0}, A\right) ; \bar{\psi}_{A}\left(u_{0}+u_{1}, t\right) ;\right. \\
\left.\psi\left(u_{0}, t\right)-\bar{\psi}(0, t-A)\left[\psi\left(u_{0}, A\right)-\psi\left(u_{0}+u_{1}, A\right)\right]\right\} \cdot(25)
\end{aligned}
$$

(ii) When considering the specific alarm time defined in (12), and with $T=T\left(u_{0}\right)$, we have,

$$
\left.\Delta(t) \leq \beta \bar{\psi}\left(u_{1}, t-A\right)\right)+\left(\psi\left(u_{1}, t-A\right)-\psi\left(u_{0}+e^{-r A} u_{1}, t\right)\right)
$$

Notice that the maximum difference between the proposed lower and upper bounds for $\Delta(t)$ is given in $(24)$ and (25).

\section{b) Ultimate time Ruin Probabilities}

Let us extend the above results for infinite time, under $N P C$, as discussed in the beginning of Section 3.1. The difference $\Delta$ between ultimate time ruin probabilities in the two models, viz. $\left(M_{r}\right)$ and $\left(M^{A}\right)$, is given under the NPC by

$$
\begin{aligned}
\Delta= & P\left[T^{M^{A}}<\infty\right]-P\left[T^{M_{r}}<\infty\right]=P\left[T^{M_{r}}=\infty\right]-P\left[T^{M^{A}}=\infty\right] \\
= & \psi\left(u_{0}, A\right)-\psi\left(u_{0}+e^{-r A} u_{1}\right)+ \\
& \quad \int_{-\infty}^{u_{0}} \psi\left(u_{0}+u_{1}-x\right) \bar{\psi}\left(u_{0}, A \mid R_{A}=x\right) d F_{R_{A}}(x) \\
= & \bar{\psi}\left(u_{0}+e^{-r A} u_{1}\right)-\int_{-\infty}^{u_{0}} \bar{\psi}\left(u_{0}+u_{1}-x\right) \bar{\psi}\left(u_{0}, A \mid R_{A}=x\right) d F_{R_{A}}(x)
\end{aligned}
$$


or, alternatively

$$
\begin{aligned}
\Delta= & \psi\left(u_{0}+u_{1}\right)-\psi\left(u_{0}+e^{-r A} u_{1}\right)+ \\
& \int_{-\infty}^{u_{0}+u_{1}} \bar{\psi}\left(u_{0}+u_{1}-x\right)\left(\psi\left(u_{0}, A \mid R_{A}=x\right)-\psi\left(u_{0}+u_{1}, A \mid R_{A}=x\right)\right) d F_{R_{A}}(x) .
\end{aligned}
$$

Moreover it has the following bounds, given in the proposition below.

Proposition 2 The difference $\Delta$ between ultimate time ruin probabilities in the two models $\left(M_{r}\right)$ and $\left(M^{A}\right)$ satisfies, under the NPC:

(i) for any deterministic alarm time A,

$$
\Delta \leq 1-\bar{\psi}\left(u_{0}, A\right) \bar{\psi}\left(u_{1}\right)-\psi\left(u_{0}+e^{-r A} u_{1}\right) \leq 1-\bar{\psi}\left(u_{0}, A\right) \bar{\psi}\left(u_{1}\right)-\psi\left(u_{0}+u_{1}\right)
$$

and

$$
\begin{aligned}
& \Delta \geq \max \left\{\psi\left(u_{0}+e^{-r A} u_{1}\right)-\min \left(\bar{\psi}\left(u_{0}, A\right), \bar{\psi}_{A}\left(u_{0}+u_{1}\right)\right) ;\right. \\
&\left.\psi\left(u_{0}+u_{1}\right)-\psi\left(u_{0}+e^{-r A} u_{1}\right)+\bar{\psi}(0)\left(\psi\left(u_{0}, A\right)-\psi\left(u_{0}+u_{1}, A\right)\right)\right\} \\
& \geq \psi\left(u_{0}+u_{1}\right)-\min \left\{\bar{\psi}\left(u_{0}, A\right) ; \bar{\psi}_{A}\left(u_{0}+u_{1}\right) ;\right. \\
&\left.\psi\left(u_{0}\right)-\psi\left(u_{0}+u_{1}\right)-\bar{\psi}(0)\left(\psi\left(u_{0}, A\right)-\psi\left(u_{0}+u_{1}, A\right)\right)\right\} .
\end{aligned}
$$

(ii) for the alarm time $A$ defined as in (12) (and with $T=T\left(u_{0}\right)$ ),

$$
\Delta \leq \beta \bar{\psi}\left(u_{1}\right)+\left(\psi\left(u_{1}\right)-\psi\left(u_{0}+e^{-r A} u_{1}\right)\right)
$$

The upper bound in (31) tells us the possible loss in terms of infinite ruin probability; indeed, the upper bound can be strictly less than $\beta \bar{\psi}\left(u_{1}\right)$ (i.e. much smaller than $\beta$, so very small) whenever $\psi\left(u_{1}\right)-\psi\left(u_{0}+e^{-r A} u_{1}\right)<0$, i.e. whenever $u_{1}\left(1-e^{-r A}\right)>u_{0}$ which is possible only if $u_{0}<u_{1}$. It means that a system with only one alarm time may at least allow to postpone of lag $A$ the fact of putting a large capital. It also means that it might be interesting to develop the study of multiple alarms to be able to put a reduced amount at each alarm time.

Note that we also have $\Delta \leq \beta \bar{\psi}\left(u_{1}\right)+\left(\psi\left(u_{1}\right)-\psi\left(u_{0}+u_{1}\right)\right)$ which is much less interesting since $\left(\psi\left(u_{1}\right)-\psi\left(u_{0}+u_{1}\right)\right)$ is always positive.

\subsection{Generalized comparison}

We now generalize the results in Section 3.1 to compare the model $M^{k}$, for any $k \geq 1$, possibly even $\infty$ (and with $M^{1}=M^{A}$ from the previous subsection), when considering an alarm system with $k$ alarm times as defined in (15) and the model $M_{r}$. We want to compare the ultimate and finite time ruin probabilities of those two models. Towards this, we propose two methods, a recursive one and a direct one.

\subsubsection{Recursive method}

In this method we artificially introduce $k-1$ intermediate models having a specific number $(1, \ldots, k-1)$ of alarms in order to use the one alarm comparison. To enable a fair comparison across the models, we consider the amount being put at the time of the alarm to be such that amounts are equivalent across the models considering the time value of money. To be specific, the model $M^{k}$ (the final model) calls for starting with initial capital $u_{0}$ and putting an amount $u_{i}$ at 
the time of the $i$-th alarm, i.e. $A_{i}, \forall i=1, \ldots, k$. On the other hand, the model preceding it, $M^{k-1}$ allows for capital change only at the time of the first $k-1$ alarms and the amount being put at all but the last of them coincides with the same for model $M^{k}$. Hence, at the time of the $(k-1)-$ th alarm in model $M^{k-1}$, the amount put is given by

$$
u_{k-1}+u_{k} \times e^{-r\left(A_{k}-A_{k-1}\right)} .
$$

Subsequent models are defined similarly. To consider a complete mathematical framework, let us identify $A_{0}=0$ (0-th alarm time) as the starting time of the process. Note that the stochastic process behind all the models remains the same as $R_{t}$; however the ruin or otherwise as per model $M^{i}$ at time $t$, depends on the level applicable at that time, dictated by the amounts added to the system, as per the model specifications. For $i=0,1, \ldots, k$, referring to Model $M^{i}$, these levels at time $t$, denoted by $l_{t}^{i}$, are given by

$$
l_{t}^{i}=\left\{\begin{array}{lc}
\sum_{j=0}^{m} u_{j} & \text { if } t \in\left(A_{m}, A_{m+1}\right] \text { for some } m<i, \\
\sum_{j=0}^{i} u_{j}+\sum_{j=i+1}^{k} u_{j} \times e^{-r\left(A_{j}-A_{i}\right)}=l_{A_{i+1}}^{i} & \text { for } t>A_{i}
\end{array}\right.
$$

Thus, for $i=0,1, \cdots, k-1$, the levels from the successive models may be compared as:

$$
l_{t}^{i}=l_{t}^{i+1}, \forall t \leq A_{i} ; \quad l_{t}^{i}>l_{t}^{i+1}, \forall t \in\left(A_{i}, A_{i+1}\right] ; \quad l_{t}^{i}<l_{t}^{i+1}, \forall t>A_{i+1} .
$$

Note that we set $A_{k+1}=\infty$ and the model $M^{0}$ corresponds to $M_{r}$ with level

$$
l_{t}^{0}=u_{0}+\sum_{j=1}^{k} u_{j} e^{-r\left(A_{j}-A_{i}\right)}, \quad \forall t>A_{0}=0 .
$$

The comparison across the consecutive levels may be better understood through the following figure: 


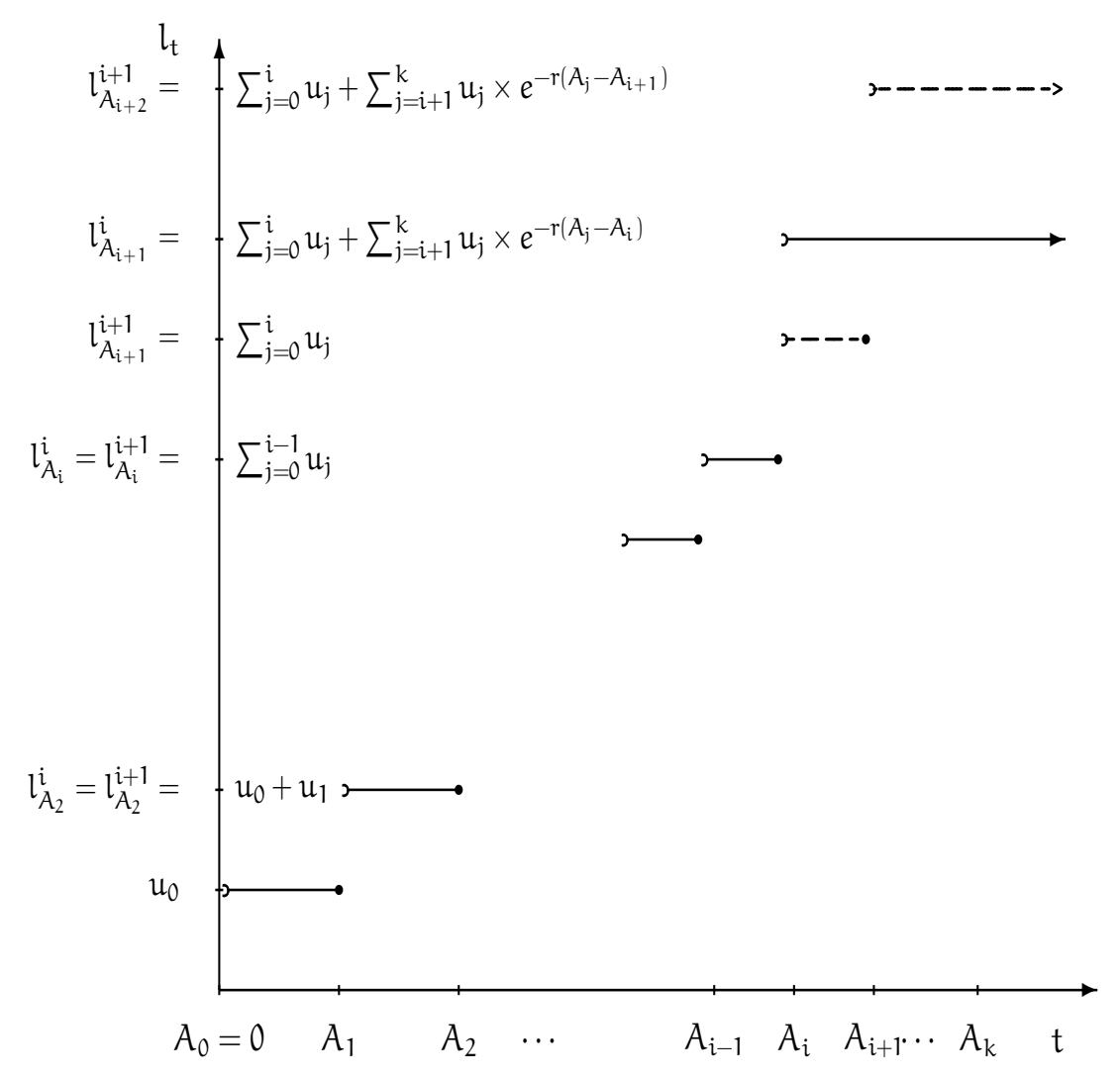

Figure 5: Capital levels under $M^{i}$ (solid line) and $M^{i+1}$ (dashed line, when different after $A_{i}$ )

\section{- Exact Evaluation over Finite and Infinite time Horizon:}

We want to evaluate:

$$
\Delta(t):=P\left(T^{M^{k}} \leq t\right)-P\left(T^{M_{r}} \leq t\right)
$$

for $t>A_{1}$. Note that,

there exists a unique $i$ with $1<i \leq k+1 \quad$ s.t. $t \in\left(A_{i-1}, A_{i}\right]$, (with $A_{k+1}=\infty$ ). (34) For $i \leq k$, i.e. for $A_{1} \leq t \leq A_{k}, \Delta(t)$ can be written as

$$
\begin{aligned}
\Delta(t) & =\sum_{j=0}^{k-1}\left(P\left[T^{M^{j}}>t\right]-P\left[T^{M^{j+1}}>t\right]\right) \\
& =\sum_{j=0}^{i-1}\left(P\left[T^{M^{j}}>t\right]-P\left[T^{M^{j+1}}>t\right]\right)+\sum_{j=i}^{k-1}\left(P\left[T^{M^{j}}>t\right]-P\left[T^{M^{j+1}}>t\right]\right) \\
& =\sum_{j=0}^{i-1}\left(P\left[T^{M^{j}}>t\right]-P\left[T^{M^{j+1}}>t\right]\right)
\end{aligned}
$$

since the models $M^{j}$ and $M^{j+1}$ have the same levels of reference until time $A_{j}$, hence until $t$, for any $j \geq i$. 
Similarly, for $t>A_{k}, \Delta(t)=\sum_{j=0}^{k-1}\left(P\left[T^{M^{j}}>t\right]-P\left[T^{M^{j+1}}>t\right]\right)$. Thus, we have

$$
\Delta(t)=\sum_{j=0}^{i-2}\left(P\left[T^{M^{j}}>t\right]-P\left[T^{M^{j+1}}>t\right]\right)+1_{(i \leq k)}\left(P\left[T^{M^{i-1}}>t\right]-P\left[T^{M^{i}}>t\right]\right) .
$$

with $i$ as defined in (34) for any $t>A_{1}$.

To compute the terms on the RHS of this last equation, we will be using that, for any $j$, the two events (no ruin for $M^{j}$ up to $A_{j}$ ) and (no ruin for $M^{j+1}$ up to $A_{j}$ ) coincide because of (33) whenever $t \leq A_{j}$. Let us denote this event by $B_{j}$, as defined in (14) that we recall here for convenience:

$$
B_{j}:=\bigcap_{n=1}^{j}\left(T\left(A_{n-1}, \sum_{m=0}^{n-1} u_{m}\right)>A_{n}\right) \text {. }
$$

Let us start to evaluate the second term on the RHS of (35). Using the above property of $B_{i-1}$, we can write

$$
\begin{aligned}
P\left[T^{M^{i-1}}>t\right]-P\left[T^{M^{i}}>t\right] & =P\left[B_{i-1} \cap\left(T\left(A_{i-1}, l_{A_{i}}^{i-1}\right)>t\right)\right]-P\left[B_{i-1} \cap\left(T\left(A_{i-1}, l_{A_{i}}^{i}\right)>t\right)\right] \\
& =P\left(B_{i-1}\right)\left[\psi_{A_{i-1}}\left(l_{A_{i}}^{i}, t \mid B_{i-1}\right)-\psi_{A_{i-1}}\left(l_{A_{i}}^{i-1}, t \mid B_{i-1}\right)\right]
\end{aligned}
$$

or, in a similar way,

$$
\begin{aligned}
P\left[T^{M^{i-1}}>t\right]-P\left[T^{M^{i}}>t\right]= & \int_{-\infty}^{l_{A_{i-1}}^{i-1}}\left\{P\left[B_{i-1} \cap\left(T\left(A_{i-1}, l_{A_{i}}^{i-1}\right)>t\right) \mid R_{A_{i-1}}=x\right]\right. \\
\left.-P\left[B_{i-1} \cap\left(T\left(A_{i-1}, l_{A_{i}}^{i}\right)>t\right) \mid R_{A_{i-1}}=x\right]\right\} d F_{R_{A_{i-1}}}(x) & \\
= & \int_{-\infty}^{l_{A_{i-1}-1}^{i-1}} P\left[B_{i-1} \mid R_{A_{i-1}}=x\right]\left\{\psi_{A_{i-1}}\left(l_{A_{i}}^{i}, t \mid B_{i-1} \cap\left(R_{A_{i-1}}=x\right)\right)\right. \\
& \left.-\psi_{A_{i-1}}\left(l_{A_{i}}^{i-1}, t \mid B_{i-1} \cap\left(R_{A_{i-1}}=x\right)\right)\right\} d F_{R_{A_{i-1}}}(x) \\
= & \int_{-\infty}^{l_{A_{i-1}}^{i-1}}\left\{\psi\left(l_{A_{i}}^{i}-x, t-A_{i-1}\right)-\psi\left(l_{A_{i}}^{i-1}-x, t-A_{i-1}\right)\right\} \\
& P\left[B_{i-1} \mid R_{A_{i-1}}=x\right] d F_{R_{A_{i-1}}}(x)
\end{aligned}
$$

using (7) in the last equality.

An upper bound can then be deduced as

$$
\begin{aligned}
& P\left[T^{M^{i-1}}>t\right]-P\left[T^{M^{i}}>t\right] \leq \max _{x \leq l_{A_{i-1}}^{i-1}}\left\{\psi\left(l_{A_{i}}^{i}-x, t-A_{i-1}\right)-\psi\left(l_{A_{i}}^{i-1}-x, t-A_{i-1}\right)\right\} \\
& \int_{-\infty}^{l_{A_{i-1}}^{i-1}} P\left[B_{i-1} \mid R_{A_{i-1}}=x\right] d F_{R_{A_{i-1}}}(x) \\
& =P\left(B_{i-1}\right) \max _{x \leq l_{A_{i-1}}^{i-1}}\left(\psi\left(l_{A_{i}}^{i}-x, t-A_{i-1}\right)-\psi\left(l_{A_{i}}^{i-1}-x, t-A_{i-1}\right)\right) \\
& \leq P\left(B_{i-1}\right) \psi\left(l_{A_{i}}^{i}-l_{A_{i-1}}^{i-1}, t-A_{i-1}\right)=P\left(B_{i-1}\right) \psi\left(u_{i-1}, t-A_{i-1}\right)
\end{aligned}
$$


whereas a lower bound is given by

$$
P\left[T^{M^{i-1}}>t\right]-P\left[T^{M^{i}}>t\right] \geq P\left(B_{i-1}\right) \min _{x \leq l_{A_{i-1}}^{-1}}\left(\psi\left(l_{A_{i}}^{i}-x, t-A_{i-1}\right)-\psi\left(l_{A_{i}}^{i-1}-x, t-A_{i-1}\right)\right) .
$$

Now let us consider the first term on the RHS of (35). Since $t>A_{i-1}$, for any $0 \leq j \leq i-2$, we have:

$P\left[T^{M^{j}}>t\right]-P\left[T^{M^{j+1}}>t\right]=P\left[\left(\right.\right.$ no ruin for $M^{j}$ up to $\left.A_{j}\right) \cap\left(\right.$ no ruin for $M^{j}$ between $A_{j}$ and $\left.\left.t\right)\right]$

$-P\left[\left(\right.\right.$ no ruin for $M^{j+1}$ up to $\left.A_{j}\right) \cap\left(\right.$ no ruin for $M^{j+1}$ between $A_{j}$ and $\left.\left.t\right)\right]$

$=\int_{-\infty}^{l_{A_{j}}^{j}}\left(P\left[\left(\right.\right.\right.$ no ruin for $M^{j}$ up to $\left.A_{j}\right) \cap\left(\right.$ no ruin for $M^{j}$ between $A_{j}$ and $\left.\left.t\right) \mid R_{A_{j}}=x\right]$

$-P\left[\left(\right.\right.$ no ruin for $M^{j+1}$ up to $\left.A_{j}\right) \cap\left(\right.$ no ruin for $M^{j+1}$ between $A_{j}$ and $\left.\left.\left.t\right) \mid R_{A_{j}}=x\right]\right) d F_{R_{A_{j}}}(x)$.

Using the same conditioning and arguments as previously, we obtain

$$
P\left[T^{M^{j}}>t\right]-P\left[T^{M^{j+1}}>t\right]=\int_{-\infty}^{l_{A_{j}}^{j}} P\left[B_{j} \mid R_{A_{j}}=x\right] \Delta^{j}(x, t) d F_{R_{A_{j}}}(x)
$$

with

$$
\begin{aligned}
\Delta^{j}(x, t):= & P\left[\left(T\left(A_{j}, l_{A_{j+1}}^{j}\right)>t\right) \mid B_{j} \cap\left(R_{A_{j}}=x\right)\right]- \\
& P\left[\left(T\left(A_{j}, l_{A_{j+1}}^{j+1}\right)>A_{j+1}\right) \cap\left(T\left(A_{j+1}, l_{A_{j+2}}^{j+1}\right)>t\right) \mid B_{j} \cap\left(R_{A_{j}}=x\right)\right] .
\end{aligned}
$$

Note that $\Delta^{j}(x, t)$, for $0 \leq j \leq i-2$, corresponds to the difference of ruin probabilities for finite time $t>A_{j+1}$ between the two models $M_{r}$ and $M^{A}$ developed in Section 3.1, when taking the time origin at $A_{j}$ with the value $R_{A_{j}}=x$ instead of $R_{0}=0$ ), the one alarm time $A_{j+1}$ instead of $A$, and the associated amounts:

$$
\begin{gathered}
l_{A_{j+1}}^{j+1}=\sum_{n=0}^{j} u_{n} \text { instead of } u_{0}, \\
l_{A_{j+1}}^{j}=\sum_{n=0}^{j} u_{n}+\sum_{n=j+1}^{k} u_{n} e^{-r\left(A_{n}-A_{j}\right)} \quad \text { instead of } u_{0}+e^{-r A} u_{1} \\
\text { and } l_{A_{j+2}}^{j+1}=\sum_{n=0}^{j+1} u_{n}+\sum_{n=j+2}^{k} u_{n} e^{-r\left(A_{n}-A_{j+1}\right)} \quad \text { instead of } u_{0}+u_{1} .
\end{gathered}
$$

Hence applying (22) under those conditions provides for $0 \leq j \leq i-2$, when shifting backward time of $A_{j}$ and levels of $x$ in the expression of $\Delta(t)$ (using the independence and stationarity of the increments of $R$ ),

$$
\begin{aligned}
& \Delta^{j}(x, t)=\psi\left(l_{A_{j+1}}^{j+1}-x, A_{j+1}-A_{j}\right)-\psi\left(l_{A_{j+1}}^{j}-x, t-A_{j}\right)+ \\
& \int_{-\infty}^{l_{A_{j+1}}^{j+1}-x} \psi\left(l_{A_{j+2}+1}^{j+1}-x-y, t-A_{j+1}\right) \bar{\psi}\left(l_{A_{j+1}^{j+1}}^{j+1}-x, A_{j+1}-A_{j} \mid R_{A_{j+1}-A_{j}}=y\right) d F_{R_{A_{j+1}-A_{j}}}(y) .
\end{aligned}
$$


Note that it can also be written as

$$
\begin{aligned}
& \Delta^{j}(x, t)= \bar{\psi}\left(l_{A_{j+1}}^{j}-x, t-A_{j}\right)-\int_{-\infty}^{l_{A_{j+1}}^{j+1}-x} \bar{\psi}\left(l_{A_{j+2}}^{j+1}-x-y, t-A_{j+1}\right) \times \\
& \bar{\psi}\left(l_{A_{j+1}+1}^{j+1}-x, A_{j+1}-A_{j} \mid R_{A_{j+1}-A_{j}}=y\right) d F_{R_{A_{j+1}-A_{j}}}(y) \\
&=\bar{\psi}\left(l_{A_{j+1}^{j}}^{j}-x, t-A_{j}\right)-\int_{-\infty}^{l_{A_{j+1}}^{j+1}} \bar{\psi}\left(l_{A_{j+2}^{j+1}}^{j+z}, t-A_{j+1}\right) \times \\
& \bar{\psi}\left(l_{A_{j+1}}^{j+1}-x, A_{j+1}-A_{j} \mid R_{A_{j+1}-A_{j}}=z-x\right) d F_{R_{A_{j+1}-A_{j}}}(z) .
\end{aligned}
$$

Equivalently, the second expression of $\Delta(x)$ given in (23) can be translated as

$$
\begin{aligned}
& \Delta^{j}(x, t)=\psi\left(l_{A_{j+2}}^{j+1}-x, t-A_{j}\right)-\psi\left(l_{A_{j+1}^{j}}^{j}-x, t-A_{j}\right)+ \\
& \int_{-\infty}^{l_{A_{j+2}}^{j+1}-x} \bar{\psi}\left(l_{A_{j+2}^{j+1}}^{j+1}-x-y, t-A_{j+1}\right)\left[\psi\left(l_{A_{j+1}^{j+1}}^{j+1}-x, A_{j+1}-A_{j} \mid R_{A_{j+1}-A_{j}}=y\right)\right. \\
& \left.\quad-\psi\left(l_{A_{j+2}}^{j+1}-x, A_{j+1}-A_{j} \mid R_{A_{j+1}-A_{j}}=y\right)\right] d F_{R_{A_{j+1}-A_{j}}}(y) .
\end{aligned}
$$

The upper bound of $\Delta^{j}(x, t)$ can be deduced from (42) or (43) in the same way as in the case of one alarm time, or translated from (24) as

$$
\begin{aligned}
\Delta^{j}(x, t) & \leq 1-\bar{\psi}\left(l_{A_{j+1}}^{j+1}-x, A_{j+1}-A_{j}\right) \bar{\psi}\left(l_{A_{j+2}+1}^{j+1}-l_{A_{j+1}}^{j+1}, t-A_{j+1}\right)-\psi\left(l_{A_{j+1}}^{j}-x, t-A_{j}\right) \\
& =\bar{\psi}\left(l_{A_{j+1}}^{j}-x, t-A_{j}\right)-\bar{\psi}\left(l_{A_{j+1}^{j+1}}-x, A_{j+1}-A_{j}\right) \bar{\psi}\left(l_{A_{j+2}}^{j+1}-l_{A_{j+1}}^{j+1}, t-A_{j+1}\right)(45)
\end{aligned}
$$

and the lower bound comes from (44) or can be translated via (25) as

$$
\begin{aligned}
\Delta^{j}(x, t) \geq & \psi\left(l_{A_{j+2}}^{j+1}-x, t-A_{j}\right)-\psi\left(l_{A_{j+1}}^{j}-x, t-A_{j}\right) \\
& +\bar{\psi}\left(0, t-A_{j+1}\right)\left[\psi\left(l_{A_{j+1}^{j+1}}^{j+1}-x, A_{j+1}-A_{j}\right)-\psi\left(l_{A_{j+2}}^{j+1}-x, A_{j+1}-A_{j}\right)\right] .
\end{aligned}
$$

Conclusion: Combining (35) with (37), (41), (43) (respectively (44)), the comparison between the two models can be evaluated for $t \in\left(A_{i-1}, A_{i}\right], 2 \leq i \leq k+1$, according to

$$
\begin{aligned}
\Delta(t)= & \sum_{j=0}^{i-2} \int_{-\infty}^{l_{A_{j}}^{j}} P\left[B_{j} \mid R_{A_{j}}=x\right]\left\{\bar{\psi}\left(l_{A_{j+1}^{j}}^{j}-x, t-A_{j}\right)-\int_{-\infty}^{l_{A_{j+1}}^{j+1}} \bar{\psi}\left(l_{A_{j+2}}^{j+1}-y, t-A_{j+1}\right)\right. \\
& \left.\times \bar{\psi}\left(l_{A_{j+1}}^{j+1}-x, A_{j+1}-A_{j} \mid R_{A_{j+1}-A_{j}}=y-x\right) d F_{R_{A_{j+1}-A_{j}}}(y)\right\} d F_{R_{A_{j}}}(x) \\
+ & 1_{(i \leq k)} \int_{-\infty}^{l_{A_{i-1}-1}^{i}}\left\{\psi\left(l_{A_{i}}^{i}-x, t-A_{i-1}\right) \psi\left(l_{A_{i}}^{i-1}-x, t-A_{i-1}\right)\right\} P\left[B_{i-1} \mid R_{A_{i-1}}=x\right] d F_{R_{A_{i-1}}}(x)
\end{aligned}
$$

or equivalently

$$
\begin{aligned}
\Delta(t)= & -\sum_{j=0}^{i-2} \int_{-\infty}^{l_{A_{j}}^{j}}\left\{\psi\left(l_{A_{j+1}}^{j}-x, t-A_{j}\right)-\psi\left(l_{A_{j+2}}^{j+1}-x, t-A_{j}\right)\right\} P\left[B_{j} \mid R_{A_{j}}=x\right] d F_{R_{A_{j}}}(x) \\
+ & \sum_{j=0}^{i-2} \int_{-\infty}^{l_{A_{j}}^{j}} P\left[B_{j} \mid R_{A_{j}}=x\right] \int_{-\infty}^{l_{A_{j+2}}^{j+1}-x}\left[\psi\left(l_{A_{j+1}}^{j+1}-x, A_{j+1}-A_{j} \mid R_{A_{j+1}-A_{j}}=y\right)-\right. \\
& \left.\psi\left(l_{A_{j+2}}^{j+1}-x, A_{j+1}-A_{j} \mid R_{A_{j+1}-A_{j}}=y\right)\right] \bar{\psi}\left(l_{A_{j+2}+1}^{j+1}-x-y, t-A_{j+1}\right) d F_{R_{A_{j+1}-A_{j}}}(y) d F_{R_{A_{j}}}(x) \\
+ & 1_{(i \leq k)} \int_{-\infty}^{l_{A_{i-1}-1}^{i-1}}\left\{\psi\left(l_{A_{i}}^{i}-x, t-A_{i-1}\right)-\psi\left(l_{A_{i}}^{i-1}-x, t-A_{i-1}\right)\right\} P\left[B_{i-1} \mid R_{A_{i-1}}=x\right] d F_{R_{A_{i-1}}}(x) .
\end{aligned}
$$


We can extend those results, either when taking the limit as $t \rightarrow \infty$ (which implies to consider $i=k+1$ in the results above), or when shifting backward time of $A_{i}$ and levels of $x$ in (27) and (28) (using the independence and stationarity of the increments of $R$ ).

The ultimate ruin probabilities can then be expressed as:

$$
\begin{gathered}
\Delta=\sum_{j=0}^{k-1} \int_{-\infty}^{l_{A_{j}}^{j}} \Delta^{j}(x) P\left[B_{j} \mid\left(R_{A_{j}}=x\right)\right] d F_{R_{A_{j}}}(x) \quad \text { with } \\
\Delta^{j}(x)=\bar{\psi}\left(l_{A_{j+1}^{j}}-x\right)-\int_{-\infty}^{l_{A_{j+1}+1}} \bar{\psi}\left(l_{A_{j+2}^{j+1}}^{j+1}-y\right) \times \\
\bar{\psi}\left(l_{A_{j+1}^{j+1}}-x, A_{j+1}-A_{j} \mid R_{A_{j+1}-A_{j}}=y-x\right) d F_{R_{A_{j+1}-A_{j}}}(y)
\end{gathered}
$$

or equivalently

$$
\begin{aligned}
\Delta^{j}(x)= & \int_{-\infty}^{l_{A_{j+2}}^{j+1}-x} \bar{\psi}\left(l_{A_{j+2}}^{j+1}-x-y\right)\left[\psi\left(l_{A_{j+1}}^{j+1}-x, A_{j+1}-A_{j} \mid R_{A_{j+1}-A_{j}}=y\right)\right. \\
& \left.-\psi\left(l_{A_{j+2}}^{j+1}-x, A_{j+1}-A_{j} \mid R_{A_{j+1}-A_{j}}=y\right)\right] d F_{R_{A_{j+1}-A_{j}}}(y) \\
& -\left(\psi\left(l_{A_{j+1}}^{j}-x\right)-\psi\left(l_{A_{j+2}}^{j+1}-x\right)\right) .
\end{aligned}
$$

\section{- Results on bounds}

Upper and lower bounds for $\Delta(t)$ and $\Delta$ are now deduced from the above results. The upper bounds for $\Delta(t)$ come when plugging (38) or (39), (41) and (45) in (35), whereas the lower bounds come when replacing (38) by (36) or (40), and (45) by (46). Regarding $\Delta$, bounds of $\Delta^{j}(x)$ can be deduced from (50), respectively (51), in the same way as in the case of one alarm time, or translated from (29), respectively (30).

Naturally these bounds will be useful only when they turn out to be less than 1 in absolute value as trivially $|\Delta(t)|<1$ as is $\Delta$.

i) Over finite time horizon, we have:

$$
\begin{aligned}
\Delta(t) \leq & \sum_{j=0}^{i-2} \int_{-\infty}^{l_{A_{j}}^{j}} P\left[B_{j} \mid R_{A_{j}}=x\right]\left\{\bar{\psi}\left(l_{A_{j+1}}^{j}-x, t-A_{j}\right)\right. \\
& \left.-\bar{\psi}\left(l_{A_{j+1}}^{j+1}-x, A_{j+1}-A_{j}\right) \bar{\psi}\left(l_{A_{j+2}}^{j+1}-l_{A_{j+1}}^{j+1}, t-A_{j+1}\right)\right\} d F_{R_{A_{j}}}(x) \\
& +1_{(i \leq k)} P\left(B_{i-1}\right) \max _{x \leq l_{A_{i-1}}^{i-1}}\left(\psi\left(l_{A_{i}}^{i}-x, t-A_{i-1}\right)-\psi\left(l_{A_{i}}^{i-1}-x, t-A_{i-1}\right)\right) \\
\leq & P\left(B_{i-1}\right) \psi\left(u_{i-1}, t-A_{i-1}\right) 1_{(i \leq k)}+\sum_{j=0}^{i-2} \int_{-\infty}^{l_{A_{j}}^{j}} P\left[B_{j} \mid R_{A_{j}}=x\right]\left\{\bar{\psi}\left(l_{A_{j+1}}^{j}-x, t-A_{j}\right)\right. \\
\leq & P\left(B_{i-1}\right) \psi\left(u_{i-1}, t-A_{i-1}\right) 1_{(i \leq k)} \\
& +\sum_{j=0}^{i-2} P\left(B_{j}\right)\left[1-\bar{\psi}\left(l_{A_{j+1}}^{j+1}-l_{A_{j}}^{j}, A_{j+1}-A_{j}\right) \bar{\psi}\left(l_{A_{j+2}}^{j+1}-l_{A_{j+1}}^{j+1}, t-A_{j+1}\right)\right] \\
\leq & P\left(B_{i-1}\right) \psi\left(u_{i-1}, A_{i}-A_{i-1}\right) 1_{(i \leq k)} \\
& +\sum_{j=0}^{i-2} P\left(B_{j}\right)\left[1-\bar{\psi}\left(l_{A_{j+1}}^{j+1}-l_{A_{j}}^{j}, A_{j+1}-A_{j}\right) \bar{\psi}\left(l_{A_{j+2}}^{j+1}-l_{A_{j+1}}^{j+1}, t-A_{j+1}\right)\right\} d F_{R_{A_{j}}}(x)
\end{aligned}
$$


or

$$
\begin{aligned}
\Delta(t) \leq & -\sum_{j=0}^{i-2} \int_{-\infty}^{l_{A_{j}}^{j}}\left(\psi\left(l_{A_{j+1}}^{j}-x, t-A_{j}\right)-\psi\left(l_{A_{j+2}}^{j+1}-x, t-A_{j}\right)\right) P\left[B_{j} \mid R_{A_{j}}=x\right] d F_{R_{A_{j}}}(x) \\
+ & \sum_{j=0}^{i-2} \int_{-\infty}^{l_{A_{j}}^{j}}\left(\psi\left(l_{A_{j+1}}^{j+1}-x, A_{j+1}-A_{j}\right)-\psi\left(l_{A_{j+2}}^{j+1}-x, A_{j+1}-A_{j}\right)\right) P\left[B_{j} \mid R_{A_{j}}=x\right] d F_{R_{A_{j}}}(x) \\
& +1_{(i \leq k)} P\left(B_{i-1}\right) \max _{x \leq l_{A_{i-1}}^{i-1}}\left(\psi\left(l_{A_{i}}^{i}-x, t-A_{i-1}\right)-\psi\left(l_{A_{i}}^{i-1}-x, t-A_{i-1}\right)\right) \\
\leq & -\sum_{j=0}^{i-2} P\left(B_{j}\right) \min _{x \leq l_{A_{j}}^{j}}\left(\psi\left(l_{A_{j+1}}^{j}-x, t-A_{j}\right)-\psi\left(l_{A_{j+2}}^{j+1}-x, t-A_{j}\right)\right) \\
& +\sum_{j=0}^{i-2} P\left(B_{j}\right) \max _{x \leq l_{A_{j}}^{j}}\left(\psi\left(l_{A_{j+1}}^{j+1}-x, A_{j+1}-A_{j}\right)-\psi\left(l_{A_{j+2}}^{j+1}-x, A_{j+1}-A_{j}\right)\right) \\
& +1_{(i \leq k)} P\left(B_{i-1}\right) \max _{x \leq l_{A_{i-1}}^{i-1}}\left(\psi\left(l_{A_{i}}^{i}-x, t-A_{i-1}\right)-\psi\left(l_{A_{i}}^{i-1}-x, t-A_{i-1}\right)\right) \\
\leq & -\sum_{j=0}^{i-2} P\left(B_{j}\right) \min _{t \in\left(A_{i-1}, A_{i}\right]_{x \leq l}} \min _{A_{j}}\left(\psi\left(l_{A_{j+1}}^{j}-x, t-A_{j}\right)-\psi\left(l_{A_{j+2}}^{j+1}-x, t-A_{j}\right)\right) \\
& +\sum_{j=0}^{i-2} P\left(B_{j}\right) \max _{x \leq l_{A_{j}}^{j}}\left(\psi\left(l_{A_{j+1}}^{j+1}-x, A_{j+1}-A_{j}\right)-\psi\left(l_{A_{j+2}}^{j+1}-x, A_{j+1}-A_{j}\right)\right) \\
& +1_{(i \leq k)} P\left(B_{i-1}\right) \max _{t \in\left(A_{i-1}, A_{i}\right]} \max _{x \leq l_{A_{i-1}}^{i-1}}\left(\psi\left(l_{A_{i}}^{i}-x, t-A_{i-1}\right)-\psi\left(l_{A_{i}}^{i-1}-x, t-A_{i-1}\right)\right)
\end{aligned}
$$




$$
\begin{aligned}
& \geq-\sum_{j=0}^{i-2} P\left(B_{j}\right) \max _{t \in\left(A_{i-1}, A_{i}\right]} \max _{x \leq l_{A_{j}}^{j}}\left(\psi\left(l_{A_{j+1}}^{j}-x, t-A_{j}\right)-\psi\left(l_{A_{j+2}}^{j+1}-x, t-A_{j}\right)\right) \\
& +\sum_{j=0}^{i-2} P\left(B_{j}\right) \bar{\psi}\left(0, A_{i}-A_{j+1}\right) \min _{x \leq l_{A_{j}}^{j}}\left[\psi\left(l_{A_{j+1}}^{j+1}-x, A_{j+1}-A_{j}\right)-\psi\left(l_{A_{j+2}}^{j+1}-x, A_{j+1}-A_{j}\right)\right] \\
& +1_{(i \leq k)} P\left(B_{i-1}\right) \min _{t \in\left(A_{i-1}, A_{i}\right]} \min _{x \leq l_{A_{i-1}}^{i-1}}\left(\psi\left(l_{A_{i}}^{i}-x, t-A_{i-1}\right)-\psi\left(l_{A_{i}}^{i-1}-x, t-A_{i-1}\right)\right) .
\end{aligned}
$$

ii) Possible bounds for $\Delta$ can be given according to

$$
\begin{aligned}
\Delta \leq & \sum_{j=0}^{k-1} \int_{-\infty}^{l_{A_{j}}^{j}} P\left[B_{j} \mid R_{A_{j}}=x\right]\left\{\bar{\psi}\left(l_{A_{j+1}}^{j}-x\right)\right. \\
& \left.-\bar{\psi}\left(l_{A_{j+1}+1}^{j+1}-x, A_{j+1}-A_{j}\right) \bar{\psi}\left(l_{A_{j+2}+1}^{j+1}-l_{A_{j+1}}^{j+1}\right)\right\} d F_{R_{A_{j}}}(x) \\
\leq & \sum_{j=0}^{k-1} \int_{-\infty}^{l_{A_{j}}^{j}} P\left[B_{j} \mid R_{A_{j}}=x\right] \bar{\psi}\left(l_{A_{j+1}}^{j}-x\right) d F_{R_{A_{j}}}(x) \\
& -\sum_{j=0}^{k-1} \bar{\psi}\left(l_{A_{j+1}}^{j+1}-l_{A_{j}}^{j}, A_{j+1}-A_{j}\right) \bar{\psi}\left(l_{A_{j+2}}^{j+1}-l_{A_{j+1}}^{j+1}\right) P\left(B_{j}\right) \\
\leq & \sum_{j=0}^{k-1} P\left(B_{j}\right)\left[1-\bar{\psi}\left(l_{A_{j+1}}^{j+1}-l_{A_{j}}^{j}, A_{j+1}-A_{j}\right) \bar{\psi}\left(l_{A_{j+2}}^{j+1}-l_{A_{j+1}}^{j+1}\right)\right]
\end{aligned}
$$

or

$$
\begin{aligned}
\Delta \leq & \sum_{j=0}^{k-1} P\left(B_{j}\right) \max _{x \leq l_{A_{j}}^{j}}\left(\psi\left(l_{A_{j+1}}^{j+1}-x, A_{j+1}-A_{j}\right)-\psi\left(l_{A_{j+2}}^{j+1}-x, A_{j+1}-A_{j}\right)\right) \\
& -\sum_{j=0}^{k-1} P\left(B_{j}\right) \min _{x \leq l_{A_{j}}^{j}}\left(\psi\left(l_{A_{j+1}}^{j}-x\right)-\psi\left(l_{A_{j+2}}^{j+1}-x\right)\right)
\end{aligned}
$$

and

$$
\begin{aligned}
\Delta \geq & \sum_{j=0}^{k-1} \int_{-\infty}^{l_{A_{j}}^{j}} P\left[B_{j} \mid R_{A_{j}}=x\right]\left\{\psi\left(l_{A_{j+2}}^{j+1}-x\right)-\psi\left(l_{A_{j+1}}^{j}-x\right)\right. \\
& \left.+\bar{\psi}(0)\left[\psi\left(l_{A_{j+1}}^{j+1}-x, A_{j+1}-A_{j}\right)-\psi\left(l_{A_{j+2}}^{j+1}-x, A_{j+1}-A_{j}\right)\right]\right\} d F_{R_{A_{j}}}(x) \\
\geq & \bar{\psi}(0) \sum_{j=0}^{k-1} P\left(B_{j}\right) \min _{x \leq l_{A_{j}}^{j}}\left(\psi\left(l_{A_{j+1}}^{j+1}-x, A_{j+1}-A_{j}\right)-\psi\left(l_{A_{j+2}}^{j+1}-x, A_{j+1}-A_{j}\right)\right) \\
& -\sum_{j=0}^{k-1} P\left(B_{j}\right) \max _{x \leq l_{A_{j}}^{j}}\left(\psi\left(l_{A_{j+1}}^{j}-x\right)-\psi\left(l_{A_{j+2}}^{j+1}-x\right)\right) .
\end{aligned}
$$

Notice that in (54), although the distance between the levels is smaller in the negative sum on $j$ than in the positive sum, it is impossible to compare the growth of those sums since $t-A_{j}>A_{j+1}-A_{j}$ (for all $0 \leq j \leq i-2$ ), unless more information on the function $\psi$ is provided.

In the following proposition, some simple bounds given above have been selected; they are expressed in terms of the function $\psi$ for which numerical computations can be performed using (10) and (11). 
Proposition 3 (i) Possible bounds for $\Delta(t)$ are given for any $t \in\left(A_{i-1}, A_{i}\right]$ by

$$
\begin{aligned}
\Delta(t) \leq & \min \left\{1 ; P\left(B_{i-1}\right) \psi\left(u_{i-1}, t-A_{i-1}\right) 1_{(i \leq k)}\right. \\
& +\sum_{j=0}^{i-2} P\left(B_{j}\right)\left[1-\bar{\psi}\left(l_{A_{j+1}}^{j+1}-l_{A_{j}}^{j}, A_{j+1}-A_{j}\right) \bar{\psi}\left(l_{A_{j+2}}^{j+1}-l_{A_{j+1}}^{j+1}, t-A_{j+1}\right)\right] \\
& -\sum_{j=0}^{i-2} P\left(B_{j}\right) \min _{x \leq l_{A_{j}}^{j}}\left(\psi\left(l_{A_{j+1}}^{j}-x, t-A_{j}\right)-\psi\left(l_{A_{j+2}}^{j+1}-x, t-A_{j}\right)\right) \\
& +\sum_{j=0}^{i-2} P\left(B_{j}\right) \max _{x \leq l_{A_{j}}^{j}}\left(\psi\left(l_{A_{j+1}}^{j+1}-x, A_{j+1}-A_{j}\right)-\psi\left(l_{A_{j+2}}^{j+1}-x, A_{j+1}-A_{j}\right)\right) \\
& \left.+1_{(i \leq k)} P\left(B_{i-1}\right) \max _{x \leq l_{A_{i-1}}^{i-1}}\left(\psi\left(l_{A_{i}}^{i}-x, t-A_{i-1}\right)-\psi\left(l_{A_{i}}^{i-1}-x, t-A_{i-1}\right)\right)\right\}
\end{aligned}
$$

and

$$
\begin{aligned}
\Delta(t) & \geq \max \left\{-1 ;-\sum_{j=0}^{i-2} P\left(B_{j}\right) \max _{x \leq l_{A_{j}}^{j}}\left(\psi\left(l_{A_{j+1}}^{j}-x, t-A_{j}\right)-\psi\left(l_{A_{j+2}}^{j+1}-x, t-A_{j}\right)\right)\right. \\
& +\sum_{j=0}^{i-2} P\left(B_{j}\right) \bar{\psi}\left(0, t-A_{j+1}\right) \min _{x \leq l_{A_{j}}^{j}}\left[\psi\left(l_{A_{j+1}^{j+1}}^{j+x}-x, A_{j+1}-A_{j}\right)-\psi\left(l_{A_{j+2}}^{j+1}-x, A_{j+1}-A_{j}\right)\right] \\
& \left.+1_{(i \leq k)} P\left(B_{i-1}\right) \min _{x \leq l_{A_{i-1}}^{i-1}}\left(\psi\left(l_{A_{i}}^{i}-x, t-A_{i-1}\right)-\psi\left(l_{A_{i}}^{i-1}-x, t-A_{i-1}\right)\right)\right\} .
\end{aligned}
$$

(ii) For $\Delta$, we have

$$
\begin{aligned}
\Delta \leq & \min \left\{1 ; \sum_{j=0}^{k-1} P\left(B_{j}\right)\left[1-\bar{\psi}\left(l_{A_{j+1}}^{j+1}-l_{A_{j}}^{j}, A_{j+1}-A_{j}\right) \bar{\psi}\left(l_{A_{j+2}}^{j+1}-l_{A_{j+1}}^{j+1}\right)\right] ;\right. \\
& \sum_{j=0}^{k-1} P\left(B_{j}\right) \max _{x \leq l_{A_{j}}^{j}}\left(\psi\left(l_{A_{j+1}}^{j+1}-x, A_{j+1}-A_{j}\right)-\psi\left(l_{A_{j+2}}^{j+1}-x, A_{j+1}-A_{j}\right)\right) \\
& \left.-\sum_{j=0}^{k-1} P\left(B_{j}\right) \min _{x \leq l_{A_{j}}^{j}}\left(\psi\left(l_{A_{j+1}}^{j}-x\right)-\psi\left(l_{A_{j+2}}^{j+1}-x\right)\right)\right\}
\end{aligned}
$$

and

$$
\begin{aligned}
\Delta \geq \max \{ & -1 ; \bar{\psi}(0) \sum_{j=0}^{k-1} P\left(B_{j}\right) \min _{x \leq l_{A_{j}}^{j}}\left(\psi\left(l_{A_{j+1}+1}^{j+1}-x, A_{j+1}-A_{j}\right)-\psi\left(l_{A_{j+2}}^{j+1}-x, A_{j+1}-A_{j}\right)\right) \\
& \left.-\sum_{j=0}^{k-1} P\left(B_{j}\right) \max _{x \leq l_{A_{j}}^{j}}\left(\psi\left(l_{A_{j+1}}^{j}-x\right)-\psi\left(l_{A_{j+2}}^{j+1}-x\right)\right)\right\} .
\end{aligned}
$$

\section{- Application to Specific Alarm Times Proposed:}

Now let us consider the alarm times proposed in (15) defined with respect to the final model $M^{k}$. Notice that the amounts involved to define those alarm times $A_{j}, 1 \leq j \leq k$, are respectively $\sum_{n=0}^{j-1} u_{n}$, for $1 \leq j \leq k$, and coincide with the levels $l_{A_{j}}^{i+1}$ whenever $j \leq i$ and with the levels $l_{A_{j}}^{i}$ when $j \leq i+1$. 
First let us compute $P\left(B_{i}\right)$, for $1 \leq i \leq k$. Definition (15) induces the recursive relation

$$
P\left(B_{i+1}\right)=P\left[T\left(A_{i}, \sum_{n=0}^{i} u_{n}\right)>A_{i+1} \mid B_{i}\right] P\left(B_{i}\right) \geq(1-\beta) P\left(B_{i}\right)
$$

which implies, for $i \geq 1$,

$$
P\left(B_{i}\right) \geq(1-\beta)^{i-1} P\left(B_{1}\right) \geq(1-\beta)^{i} ;
$$

nevertheless, because of (15), we will often consider that

$$
P\left(B_{i}\right) \simeq(1-\beta)^{i}
$$

Then notice that we have for any $t>A_{i}$ and any amount $u$,

$$
\begin{aligned}
\psi_{A_{i}}\left(u, t \mid B_{i}\right) & =\int_{-\infty}^{u} P\left[\left(\sup _{A_{i}<s \leq t} R_{s}>u\right) \mid\left(R_{A_{i}}=x\right) \cap B_{i}\right] d F_{R_{A_{i}}}(x) \\
& =\int_{-\infty}^{u} P\left[\sup _{A_{i}<s \leq t} R_{s}>u \mid B_{i} \cap\left(R_{A_{i}}=x\right)\right] P\left[B_{i} \mid R_{A_{i}}=x\right] d F_{R_{A_{i}}}(x) \\
& =\int_{-\infty}^{u} P\left[\sup _{A_{i}<s \leq t} R_{s}-R_{A_{i}}>u-x\right] P\left[B_{i} \mid R_{A_{i}}=x\right] d F_{R_{A_{i}}}(x) \\
& =\int_{-\infty}^{u} \psi\left(u-x, t-A_{i}\right) P\left[B_{i} \mid R_{A_{i}}=x\right] d F_{R_{A_{i}}}(x)
\end{aligned}
$$

from which we deduce another expression for (15), namely

$$
\begin{aligned}
& A_{i+1}=\inf \left\{s>A_{i}:\right. \\
& \int_{-\infty}^{l_{A_{i}}^{i}}\left[\psi\left(\sum_{n=0}^{i} u_{n}-x, s-A_{i}+d\right)-\psi\left(\sum_{n=0}^{i} u_{n}-x, s-A_{i}\right)\right] P\left[B_{i} \mid R_{A_{i}}=x\right] d F_{R_{A_{i}}}(x) \\
& \geq(1-\alpha) \int_{-\infty}^{l^{i} A_{i}} \bar{\psi}\left(\sum_{n=0}^{i} u_{n}-x, s-A_{i}\right) P\left[B_{i} \mid R_{A_{i}}=x\right] d F_{R_{A_{i}}}(x) \\
& \text { and } \left.\int_{-\infty}^{l_{A_{i}}^{i}} \bar{\psi}\left(\sum_{n=0}^{i} u_{n}-x, s-A_{i}\right) P\left[B_{i} \mid R_{A_{i}}=x\right] d F_{R_{A_{i}}}(x) \geq(1-\beta) P\left(B_{i}\right)\right\} .
\end{aligned}
$$

Therefore we will also often use the following approximation:

$$
\int_{-\infty}^{l_{A_{i}}^{i}} \bar{\psi}\left(l_{A_{i+1}}^{i+1}-x, A_{i+1}-A_{i}\right) P\left[B_{i} \mid R_{A_{i}}=x\right] d F_{R_{A_{i}}}(x) \simeq(1-\beta) P\left(B_{i}\right) .
$$

$\triangleright$ Bounds for $\Delta(t), t \in\left(A_{i-1}, A_{i}\right],(2 \leq i \leq k+1)$. Let us look for lower bounds of $\Delta(t)$. Using the monotonicity of $\psi$ and (40), a lower bound follows from (48), namely

$$
\begin{aligned}
\Delta(t) \geq & \sum_{j=0}^{i-2} \int_{-\infty}^{l_{A_{j}}^{j}} P\left[B_{j} \mid R_{A_{j}}=x\right]\left\{-\left[\psi\left(l_{A_{j+1}}^{j}-x, t-A_{j}\right)-\psi\left(l_{A_{j+2}}^{j+1}-x, t-A_{j}\right)\right]\right. \\
& \left.+\bar{\psi}\left(0, t-A_{j+1}\right)\left[\psi\left(l_{A_{j+1}}^{j+1}-x, A_{j+1}-A_{j}\right)-\psi\left(l_{A_{j+2}}^{j+1}-x, A_{j+1}-A_{j}\right)\right]\right\} d F_{R_{A_{j}}}(x) \\
& +1_{(i \leq k)} P\left(B_{i-1}\right) \min _{x \leq l_{A_{i-1}}^{i-1}}\left(\psi\left(l_{A_{i}}^{i}-x, t-A_{i-1}\right)-\psi\left(l_{A_{i}}^{i-1}-x, t-A_{i-1}\right)\right) .
\end{aligned}
$$


But we can write via (64) and the monotonicity of $\psi$,

$$
\begin{aligned}
& \int_{-\infty}^{l_{A_{j}}^{j}} P\left[B_{j} \mid R_{A_{j}}=x\right]\left[\psi\left(l_{A_{j+1}}^{j+1}-x, A_{j+1}-A_{j}\right)-\psi\left(l_{A_{j+2}}^{j+1}-x, A_{j+1}-A_{j}\right)\right] d F_{R_{A_{j}}}(x) \\
\geq & \int_{-\infty}^{l_{A_{j}}^{j}} P\left[B_{j} \mid R_{A_{j}}=x\right]\left[\bar{\psi}\left(l_{A_{j+2}}^{j+1}-l_{A_{j}}^{j}, A_{j+1}-A_{j}\right)-\bar{\psi}\left(l_{A_{j+1}}^{j+1}-x, A_{j+1}-A_{j}\right)\right] d F_{R_{A_{j}}}(x) \\
\simeq & P\left(B_{j}\right)\left(\bar{\psi}\left(l_{A_{j+2}}^{j+1}-l_{A_{j}}^{j}, A_{j+1}-A_{j}\right)-(1-\beta)\right)=P\left(B_{j}\right)\left(\beta-\psi\left(l_{A_{j+2}}^{j+1}-l_{A_{j}}^{j}, A_{j+1}-A_{j}\right)\right)
\end{aligned}
$$

and using also (16),

$$
\begin{gathered}
\int_{-\infty}^{l_{A_{j}}^{j}}\left(\psi\left(l_{A_{j+1}}^{j}-x, t-A_{j}\right)-\psi\left(l_{A_{j+2}}^{j+1}-x, t-A_{j}\right)\right) P\left[B_{j} \mid R_{A_{j}}=x\right] d F_{R_{A_{j}}}(x) \\
\leq \int_{-\infty}^{l_{A_{j}}^{j}} \bar{\psi}\left(l_{A_{j+1}}^{j+1}-x, A_{j+1}-A_{j}\right) P\left[B_{j} \mid R_{A_{j}}=x\right] d F_{R_{A_{j}}}(x)-\bar{\psi}\left(l_{A_{j+1}}^{j}-l_{A_{j}}^{j}, t-A_{j}\right) P\left(B_{j}\right) \\
-(1-\alpha)(1-\beta) P\left(B_{j}\right)+\max _{x \leq l_{A_{j}}^{j}}\left(\psi\left(l_{A_{j+1}}^{j+1}-x, A_{j+1}-A_{j}+d\right)-\psi\left(l_{A_{j+2}}^{j+1}-x, t-A_{j}\right)\right) P\left(B_{j}\right) \\
\simeq \quad P\left(B_{j}\right)\left[1-\beta-\bar{\psi}\left(l_{A_{j+1}}^{j}-l_{A_{j}}^{j}, t-A_{j}\right)-(1-\alpha)(1-\beta)\right. \\
+\max _{x \leq l_{A_{j}}^{j}}\left(\psi\left(l_{A_{j+1}}^{j+1}-x, A_{j+1}-A_{j}+d\right)-\psi\left(l_{A_{j+2}}^{j+1}-x, t-A_{j}\right)\right] \\
\quad\left[\alpha(1-\beta)-\bar{\psi}\left(l_{A_{j+1}}^{j}-l_{A_{j}}^{j}, t-A_{j}\right)\right. \\
\left.\quad+\max _{x \leq l_{A_{j}}^{j}}\left(\psi\left(l_{A_{j+1}^{j+1}}^{j+1}-x, A_{j+1}-A_{j}+d\right)-\psi\left(l_{A_{j+2}}^{j+1}-x, t-A_{j}\right)\right)\right] P\left(B_{j}\right),
\end{gathered}
$$

hence (55) becomes

$$
\begin{aligned}
\Delta(t) \geq & \sum_{j=0}^{i-2} P\left(B_{j}\right)\left\{-\alpha+\beta\left(\alpha+\bar{\psi}\left(0, t-A_{j+1}\right)\right)-\bar{\psi}\left(0, t-A_{j+1}\right) \psi\left(l_{A_{j+2}}^{j+1}-l_{A_{j}}^{j}, A_{j+1}-A_{j}\right)\right. \\
& \left.+\bar{\psi}\left(l_{A_{j+1}^{j}}-l_{A_{j}}^{j}, t-A_{j}\right)-\max _{x \leq l_{A_{j}}^{j}}\left(\psi\left(l_{A_{j+1}^{j+1}}^{j+1}-x, A_{j+1}-A_{j}+d\right)-\psi\left(l_{A_{j+2}}^{j+1}-x, t-A_{j}\right)\right)\right\} \\
& +1_{(i \leq k)} P\left(B_{i-1}\right) \min _{x \leq l_{A_{i-1}}^{i-1}}\left(\psi\left(l_{A_{i}}^{i}-x, t-A_{i-1}\right)-\psi\left(l_{A_{i}}^{i-1}-x, t-A_{i-1}\right)\right) \\
\geq & \sum_{j=0}^{i-2} P\left(B_{j}\right)\left\{-\alpha+\beta\left(\alpha+\bar{\psi}\left(0, t-A_{j+1}\right)\right)-\bar{\psi}\left(0, t-A_{j+1}\right) \psi\left(0, A_{j+1}-A_{j}\right)\right. \\
& \quad+1_{(i \leq k)} P\left(B_{i-1}\right) \min _{x \leq l_{A_{i-1}}^{i-1}}\left(\psi\left(l_{A_{i}}^{i}-x, t-A_{i-1}\right)-\psi\left(l_{A_{i}}^{i-1}-x, t-A_{i-1}\right)\right) \\
\geq & \left\{-\alpha+\beta(\alpha+\bar{\psi}(0, t))-\bar{\psi}\left(0, t-A_{i-1}\right) \psi\left(0, \delta_{i}^{A}\right)+\bar{\psi}(0, t)-\psi\left(0, \delta_{i}^{A}+d\right)\right\} \sum_{j=0}^{i-2} P\left(B_{j}\right) \\
& +1_{(i \leq k)} P\left(B_{i-1}\right) \min _{x \leq l_{A_{i-1}}^{i-1}}\left(\psi\left(l_{A_{i}}^{i}-x, t-A_{i-1}\right)-\psi\left(l_{A_{i}}^{i-1}-x, t-A_{i-1}\right)\right)
\end{aligned}
$$


and using (61), this last lower bound in (68) approximately equals

$$
\begin{aligned}
\{-\alpha & \left.+\beta(\alpha+\bar{\psi}(0, t))+\bar{\psi}(0, t)-\psi\left(0, \delta_{i}^{A}\right) \bar{\psi}\left(0, t-A_{i-1}\right)-\psi\left(0, \delta_{i}^{A}+d\right)\right\} \frac{1-(1-\beta)^{i-1}}{\beta} \\
& +1_{(i \leq k)}(1-\beta)^{i-1} \min _{x \leq l_{A_{i-1}^{i-1}}}\left(\psi\left(l_{A_{i}}^{i}-x, t-A_{i-1}\right)-\psi\left(l_{A_{i}}^{i-1}-x, t-A_{i-1}\right)\right)
\end{aligned}
$$

$$
\text { where } \delta_{i}^{A}:=\max _{0 \leq j \leq i-2}\left(A_{j+1}-A_{j}\right)
$$

using (61) for this last approximation.

Now let us look for possible upper bounds of $\Delta(t)$. The integral in $x$ appearing in the second sum of (48) can be bounded by

$$
\begin{aligned}
& \int_{-\infty}^{l_{A_{j}}^{j}}\left(\psi\left(l_{A_{j+1}}^{j+1}-x, A_{j+1}-A_{j}\right)-\psi\left(l_{A_{j+2}}^{j+1}-x, A_{j+1}-A_{j}\right)\right) P\left[B_{j} \mid R_{A_{j}}=x\right] d F_{R_{A_{j}}}(x) \\
& =\int_{-\infty}^{l_{A_{j}}^{j}}\left(\psi\left(l_{A_{j+1}}^{j+1}-x, A_{j+1}-A_{j}\right)-\psi\left(l_{A_{j+1}}^{j+1}-x, A_{j+1}-A_{j}+d\right)\right) P\left[B_{j} \mid R_{A_{j}}=x\right] d F_{R_{A_{j}}}(x) \\
& +\int_{-\infty}^{l_{A_{j}}^{j}}\left(\psi\left(l_{A_{j+1}}^{j+1}-x, A_{j+1}-A_{j}+d\right)-\psi\left(l_{A_{j+2}}^{j+1}-x, A_{j+1}-A_{j}\right)\right) P\left[B_{j} \mid R_{A_{j}}=x\right] d F_{R_{A_{j}}}(x)
\end{aligned}
$$

which, combined with (48), (63) and (38), provides

$$
\begin{aligned}
& \Delta(t) \leq-\sum_{j=0}^{i-2} \int_{-\infty}^{l_{A_{j}}^{j}}\left(\psi\left(l_{A_{j+1}}^{j}-x, t-A_{j}\right)-\psi\left(l_{A_{j+2}}^{j+1}-x, t-A_{j}\right)\right) P\left[B_{j} \mid R_{A_{j}}=x\right] d F_{R_{A_{j}}}(x) \\
& +\sum_{j=0}^{i-2} \int_{-\infty}^{l_{A_{j}}^{j}}\left(\psi\left(l_{A_{j+1}}^{j+1}-x, A_{j+1}-A_{j}+d\right)-\psi\left(l_{A_{j+2}}^{j+1}-x, A_{j+1}-A_{j}\right)\right) P\left[B_{j} \mid R_{A_{j}}=x\right] d F_{R_{A_{j}}}(x) \\
& -(1-\alpha)(1-\beta) \sum_{j=0}^{i-2} P\left(B_{j}\right) \\
& +1_{(i \leq k)} P\left(B_{i-1}\right) \max _{x \leq l_{A_{i-1}}^{i-1}}\left(\psi\left(l_{A_{i}}^{i}-x, t-A_{i-1}\right)-\psi\left(l_{A_{i}}^{i-1}-x, t-A_{i-1}\right)\right) \\
& \leq-(1-\alpha)(1-\beta) \sum_{j=0}^{i-2} P\left(B_{j}\right)-\sum_{j=0}^{i-2} P\left(B_{j}\right) \min _{x \leq l_{A_{j}}^{j}}\left(\psi\left(l_{A_{j+1}}^{j}-x, t-A_{j}\right)-\psi\left(l_{A_{j+2}}^{j+1}-x, t-A_{j}\right)\right) \\
& +\sum_{j=0}^{i-2} P\left(B_{j}\right) \max _{x \leq l_{A_{j}}^{j}}\left(\psi\left(l_{A_{j+1}}^{j+1}-x, A_{j+1}-A_{j}+d\right)-\psi\left(l_{A_{j+2}}^{j+1}-x, A_{j+1}-A_{j}\right)\right) \\
& +1_{(i \leq k)} P\left(B_{i-1}\right) \max _{x \leq l_{A_{i-1}^{i-1}}}\left(\psi\left(l_{A_{i}}^{i}-x, t-A_{i-1}\right)-\psi\left(l_{A_{i}}^{i-1}-x, t-A_{i-1}\right)\right) \\
& \leq\left\{-(1-\alpha)(1-\beta)-\min _{0 \leq j \leq i-2} \min _{x \leq l_{A_{j}}^{j}}\left(\psi\left(l_{A_{j+1}}^{j}-x, t-A_{j}\right)-\psi\left(l_{A_{j+2}}^{j+1}-x, t-A_{j}\right)\right)\right. \\
& \left.+\max _{0 \leq j \leq i-2} \max _{x \leq l_{A_{j}}^{j}}\left(\psi\left(l_{A_{j+1}}^{j+1}-x, A_{j+1}-A_{j}+d\right)-\psi\left(l_{A_{j+2}}^{j+1}-x, A_{j+1}-A_{j}\right)\right)\right\} \sum_{j=0}^{i-2} P\left(B_{j}\right) \\
& +1_{(i \leq k)} P\left(B_{i-1}\right) \max _{x \leq l_{A_{i-1}^{i-1}}}\left(\psi\left(l_{A_{i}}^{i}-x, t-A_{i-1}\right)-\psi\left(l_{A_{i}}^{i-1}-x, t-A_{i-1}\right)\right)
\end{aligned}
$$




$$
\begin{aligned}
& \simeq \quad\left\{-(1-\alpha)(1-\beta)-\min _{0 \leq j \leq i-2} \min _{x \leq l_{A_{j}}^{j}}\left(\psi\left(l_{A_{j+1}}^{j}-x, t-A_{j}\right)-\psi\left(l_{A_{j+2}}^{j+1}-x, t-A_{j}\right)\right)\right. \\
& \left.\quad+\max _{0 \leq j \leq i-2} \max _{x \leq l_{A_{j}}^{j}}\left(\psi\left(l_{A_{j+1}}^{j+1}-x, A_{j+1}-A_{j}+d\right)-\psi\left(l_{A_{j+2}}^{j+1}-x, A_{j+1}-A_{j}\right)\right)\right\} \frac{1-(1-\beta)^{i-1}}{\beta} \\
& \quad+1_{(i \leq k)}(1-\beta)^{i-1} \max _{x \leq l_{A_{i-1}}^{i-1}}\left(\psi\left(l_{A_{i}}^{i}-x, t-A_{i-1}\right)-\psi\left(l_{A_{i}}^{i-1}-x, t-A_{i-1}\right)\right) \\
& \leq(\beta+\alpha(1-\beta)) \frac{1-(1-\beta)^{i-1}}{\beta}+1_{(i \leq k)}(1-\beta)^{i-1} \max _{x \leq l_{A_{i-1}}^{i-1}}\left(\psi\left(l_{A_{i}}^{i}-x, t-A_{i-1}\right)-\psi\left(l_{A_{i}}^{i-1}-x, t-A_{i-1}\right)\right) .
\end{aligned}
$$

Note that, for $2 \leq i \leq k<+\infty$, this upper bound is equivalent, as $\beta \rightarrow 0$ such that $\beta(i-1)<1$, to

$$
\alpha(i-1)+(1-(i-1) \beta) \max _{x \leq l_{A_{i-1}}^{i-1}}\left(\psi\left(l_{A_{i}}^{i}-x, t-A_{i-1}\right)-\psi\left(l_{A_{i}}^{i-1}-x, t-A_{i-1}\right)\right),
$$

that has to be compared with 1 ( since $\Delta(t) \leq 1)$.

An alternative way consists in introducing the non negative parameter function of $t$ :

$$
\gamma(t):=\min _{0 \leq j \leq i-2} \min _{x \leq l_{A_{j}}^{j}} \frac{\psi\left(l_{A_{j+2}}^{j+1}-x, A_{j+1}-A_{j}\right)+\psi\left(l_{A_{j+1}}^{j}-x, t-A_{j}\right)}{\psi\left(l_{A_{j+1}}^{j+1}-x, A_{j+1}-A_{j}\right)} \geq 0
$$

such that for all $x \leq l_{A_{j}}^{j}$,

$$
\left[\psi\left(l_{A_{j+2}}^{j+1}-x, A_{j+1}-A_{j}\right)+\psi\left(l_{A_{j+1}}^{j}-x, t-A_{j}\right)\right] \geq \gamma(t) \psi\left(l_{A_{j+1}}^{j+1}-x, A_{j+1}-A_{j}\right)
$$

that might provide a smaller upper bound of $\Delta(t)$ whenever $\gamma(t)>1$.

Indeed, we deduce from (71) and (75) that

$$
\begin{aligned}
\Delta(t) \leq & 1_{(i \leq k)} P\left(B_{i-1}\right) \max _{x \leq l_{A_{i-1}}^{i-1}}\left(\psi\left(l_{A_{i}}^{i}-x, t-A_{i-1}\right)-\psi\left(l_{A_{i}}^{i-1}-x, t-A_{i-1}\right)\right) \\
& -(1-\alpha)(1-\beta) \sum_{j=0}^{i-2} P\left(B_{j}\right) \\
+ & \sum_{j=0}^{i-2}\left(\left[\psi\left(l_{A_{j+2}}^{j+1}-l_{A_{j}}^{j}, t-A_{j}\right)+\psi\left(l_{A_{j+1}}^{j+1}-l_{A_{j}}^{j}, A_{j+1}-A_{j}+d\right)\right] P\left(B_{j}\right)\right. \\
& \left.-\gamma(t) \int_{-\infty}^{l_{A_{j}}^{j}} \psi\left(l_{A_{j+1}}^{j+1}-x, A_{j+1}-A_{j}\right) P\left[B_{j} \mid R_{A_{j}}=x\right] d F_{R_{A_{j}}}(x)\right) \\
\leq & 1_{(i \leq k)} P\left(B_{i-1}\right) \max _{x \leq l_{A_{i-1}}^{i-1}}\left(\psi\left(l_{A_{i}}^{i}-x, t-A_{i-1}\right)-\psi\left(l_{A_{i}}^{i-1}-x, t-A_{i-1}\right)\right) \\
& -(1-\alpha+\gamma(t))(1-\beta) \sum_{j=0}^{i-2} P\left(B_{j}\right) \\
& +\sum_{j=0}^{i-2} P\left(B_{j}\right)\left[\psi\left(l_{A_{j+2}}^{j+1}-l_{A_{j}}^{j}, t-A_{j}\right)+\psi\left(l_{A_{j+1}}^{j+1}-l_{A_{j}}^{j}, A_{j+1}-A_{j}+d\right)\right]
\end{aligned}
$$




$$
\begin{aligned}
\leq & 1_{(i \leq k)} P\left(B_{i-1}\right) \max _{x \leq l_{A_{i-1}}^{i-1}}\left(\psi\left(l_{A_{i}}^{i}-x, t-A_{i-1}\right)-\psi\left(l_{A_{i}}^{i-1}-x, t-A_{i-1}\right)\right) \\
& +(2-(1-\alpha+\gamma(t))(1-\beta)) \sum_{j=0}^{i-2} P\left(B_{j}\right) \\
\simeq & 1_{(i \leq k)}(1-\beta)^{i-1} \max _{x \leq l_{A_{i-1}-1}^{i-1}}\left(\psi\left(l_{A_{i}}^{i}-x, t-A_{i-1}\right)-\psi\left(l_{A_{i}}^{i-1}-x, t-A_{i-1}\right)\right) \\
& +(1+\alpha-\gamma(t)+\beta(1-\alpha+\gamma(t))) \frac{1}{\beta}\left(1-(1-\beta)^{i-1}\right) .
\end{aligned}
$$

We conclude with the following proposition.

Proposition 4 The difference in the finite time ruin probabilities of the two models $M^{k}$ (with $k$ alarm times) and $M_{r}$, without alarm system but with higher equivalent initial capital, are bounded below and above by different expressions as given from (55) to (69) and from (71) to (77), respectively. These bounds, specially the lower bounds are useful in measuring effecting of the alarm system. In particular, this difference $\Delta(t)=\Delta_{k, r}(t)=P\left(T^{M^{k}} \leq t\right)-P\left(T^{M_{r}} \leq t\right)$ satisfies (72), (76) and (67), $B_{j}$ being defined in (14), namely

$$
\begin{aligned}
& \Delta(t) \leq 1_{(i \leq k)} P\left(B_{i-1}\right) \max _{x \leq l_{A_{i-1}}^{i-1}}\left(\psi\left(l_{A_{i}}^{i}-x, t-A_{i-1}\right)-\psi\left(l_{A_{i}}^{i-1}-x, t-A_{i-1}\right)\right) \\
& -(1-\alpha)(1-\beta) \sum_{j=0}^{i-2} P\left(B_{j}\right)+\min \left\{-(1-\beta) \gamma(t) \sum_{j=0}^{i-2} P\left(B_{j}\right)+\right. \\
& \sum_{j=0}^{i-2}\left[\psi\left(l_{A_{j+2}}^{j+1}-l_{A_{j}}^{j}, t-A_{j}\right)+\psi\left(l_{A_{j+1}}^{j+1}-l_{A_{j}}^{j}, A_{j+1}-A_{j}+d\right)\right] P\left(B_{j}\right) ; \\
& \sum_{j=0}^{i-2}\left[\max _{x \leq l_{A_{j}}^{j}}\left(\psi\left(l_{A_{j+1}}^{j+1}-x, A_{j+1}-A_{j}+d\right)-\psi\left(l_{A_{j+2}}^{j+1}-x, A_{j+1}-A_{j}\right)\right)\right. \\
& \left.\left.-\min _{x \leq l_{A_{j}}^{j}}\left(\psi\left(l_{A_{j+1}}^{j}-x, t-A_{j}\right)-\psi\left(l_{A_{j+2}^{j+1}}^{j+1}-x, t-A_{j}\right)\right)\right] P\left(B_{j}\right)\right\} \\
& \text { where } \gamma(t)=\min _{0 \leq j \leq i-2} \min _{x \leq l_{A_{j}}^{j}} \frac{\psi\left(l_{A_{j+2}}^{j+1}-x, A_{j+1}-A_{j}\right)+\psi\left(l_{A_{j+1}}^{j}-x, t-A_{j}\right)}{\psi\left(l_{A_{j+1}}^{j+1}-x, A_{j+1}-A_{j}\right)} \text {, }
\end{aligned}
$$

and

$$
\begin{aligned}
\Delta(t) \geq & 1_{(i \leq k)} P\left(B_{i-1}\right) \min _{x \leq l_{A_{i-1}}^{i-1}}\left(\psi\left(l_{A_{i}}^{i}-x, t-A_{i-1}\right)-\psi\left(l_{A_{i}}^{i-1}-x, t-A_{i-1}\right)\right) \\
& +\sum_{j=0}^{i-2} P\left(B_{j}\right)\left[-\alpha(1-\beta)+\left\{\beta-\psi\left(0, A_{j+1}-A_{j}\right)\right\} \bar{\psi}\left(0, t-A_{j+1}\right)\right. \\
& \left.+\bar{\psi}\left(u_{j}, t-A_{j}\right)-\psi\left(u_{j}, A_{j+1}-A_{j}+d\right)\right] .
\end{aligned}
$$

\section{Remarks.}

i) The proposition recalls explicitly some of the bounds which are easy to compute numerically, even though these are not the sharpest ones proposed. The sharper upper bound could be either of the two explicit expressions, depending on time horizon $t$ considered. Note that several alternative bounds have been provided from (55) to (77); in practice one has to choose 
the sharpest one, depending on the feasibility of the computations involved, as applicable with the available information. Of course these bounds are useful only when they are strictly less than 1 in absolute value, since $-1 \leq \Delta(t) \leq 1$. We pay greater emphasis on the upper bounds, as these provide indications when the alarm system could be competitive.

ii) The approximation $P\left(B_{j}\right) \sim(1-\beta)^{j}$ (see (61)) can be used to simplify computations (e.g. whenever there are many alarms), and leads respectively to the bounds (69) and (77).

iii) The bounds given in Proposition 4 are valid even for $t \leq A_{1}$, i.e. the case $i=1$; naturally the last terms (summation over $j$ indices) contribute nothing in that case.

iv) The third term (sum) of the upper bound not involving $\gamma(t)$ can further be simplified by ignoring the min term as for $x \approx 0$, the difference in the two terms is often very small (typically significant only at the third decimals, as to be seen in the numerical example). Consequently the difference in the upper bound, not involving $\gamma(t)$, appears only through the first term.

Corollary 1 For $1<i \leq k<+\infty$ and for $\beta \sim 0$ such that $\beta(i-1)<1$, the bounds proposed for $\Delta(t)$ can be approximated as

$$
\begin{aligned}
& \Delta(t) \leq(i-1)(\alpha+\min \{0 ; 1-\gamma(t)+\beta(1+\gamma(t)-\alpha)\})+ \\
& (1-\beta(i-1)) \max _{x \leq l_{A_{i-1}}^{i-1}}\left(\psi\left(l_{A_{i}}^{i}-x, t-A_{i-1}\right)-\psi\left(l_{A_{i}}^{i-1}-x, t-A_{i-1}\right)\right) \\
& \text { and } \Delta(t) \geq(i-1)\left(-\alpha+\bar{\psi}(0, t)-\psi\left(0, \delta_{i}^{A}\right) \bar{\psi}\left(0, t-A_{i-1}\right)-\psi\left(0, \delta_{i}^{A}+d\right)\right)+ \\
& \min _{x \leq l_{A_{i-1}}^{i-1}}\left(\psi\left(l_{A_{i}}^{i}-x, t-A_{i-1}\right)-\psi\left(l_{A_{i}}^{i-1}-x, t-A_{i-1}\right)\right) \\
& \text { with } \delta_{i}^{A} \text { defined in (70). }
\end{aligned}
$$

Note that this last upper bound decreases whenever $\gamma(t)$ increases. In particular, if $\gamma(t)$ is larger than 1, it becomes closer to 0 and might become negative meaning that the probability of survival for an alarm system might become higher than the one for a system without alarms.

Example 2. Let us revisit Example 2 to obtain a numerical evaluation of those bounds. Choosing for instance $\beta=0.225, \alpha=0.45, d=1.0$ and initial capital $u_{0}=50$ with $10 \%$ of it being added at each alarm time, we obtain exactly $k=4$ alarms occurring respectively at $A_{1}=0.29, A_{2}=0.58$, $A_{3}=0.9$ and $A_{4}=1.28$. Also consider, for example, the rate of interest $r$ to be $=10 \%$.

Evaluating the bounds for $\Delta(t)$ at different times $t_{i}$, while choosing $A_{i}<t_{i}<A_{i+1}$, for $i=1, \cdots, 4$ (with $A_{5}=\infty$ ), we observe that the upper bound of $\Delta(t)$, as given explicitly in Proposition 4 , is between 0.30 and 0.48. It is encouraging to observe that the upper bound, even though not the sharpest one, is much smaller than 1 providing indication that the alarm system is competitive in broad generality. This is reinforced through exact numerical evaluation in specific instances, narrated in Section 3.3. Below we report the detailed calculation in support.

Table 7.1

\begin{tabular}{||c|c|c|c||c|c|c|c|c|c||}
\hline Alarm No. & Alarm time & Prob of survival & \multicolumn{1}{c||}{ Cum. prob } & \multicolumn{4}{|c||}{$l_{A_{j}}^{i}$ : level at $t=A_{j}$ with $i$ alarms } \\
\hline$i$ & $A_{i}$ & $P\left(B_{i}\right)$ & $\sum_{j=0}^{i} P\left(B_{j}\right)$ & $A_{0}$ & $A_{1}$ & $A_{2}$ & $A_{3}$ & $A_{4}$ & $A_{5}$ \\
\hline 0 & 0 & 1 & 1 & 68.54 & 68.54 & 68.54 & 68.54 & 68.54 & 68.54 \\
\hline 1 & 0.29 & 0.847 & 1.847 & 50.00 & 50.00 & 69.08 & 69.08 & 69.08 & 69.08 \\
\hline 2 & 0.58 & 0.703 & 2.551 & 50.00 & 50.00 & 55.00 & 69.50 & 69.50 & 69.50 \\
\hline 3 & 0.91 & 0.566 & 3.117 & 50.00 & 50.00 & 55.00 & 60.00 & 69.82 & 69.82 \\
\hline 4 & 1.28 & 0.442 & 3.559 & 50.00 & 50.00 & 55.00 & 60.00 & 65.00 & 70.00 \\
\hline
\end{tabular}


Details for upper bound: At $t \in\left(A_{1}, A_{2}\right]$ or $i=2, P\left(B_{1}\right)=0.847$, the first term in the upper bound, as given in Proposition 4 is

$$
\begin{gathered}
1_{(i \leq k)} P\left(B_{i-1}\right) \max _{x \leq l_{A_{i-1}}^{i-1}}\left(\psi\left(l_{A_{i}}^{i}-x, t-A_{i-1}\right)-\psi\left(l_{A_{i}}^{i-1}-x, t-A_{i-1}\right)\right) \\
0.847 \times \max _{x \leq 50}(\psi(55-x, 0.11)-\psi(69.08-x, 0.11))=0.847 \times 0.178=0.138,
\end{gathered}
$$

with some details of the maximum being shown in the table below:

Table 7.2

\begin{tabular}{|l|l|l|l|l|l|l|}
\hline $\mathrm{x}$ & 0 & 10 & 20 & 30 & 40 & 50 \\
\hline$\psi(55-x, 0.11)$ & 0.050 & 0.061 & 0.077 & 0.104 & 0.157 & 0.308 \\
\hline$\psi(69.08-x, 0.11)$ & 0.041 & 0.047 & 0.056 & 0.069 & 0.091 & 0.130 \\
\hline difference & 0.010 & 0.014 & 0.021 & 0.034 & 0.066 & 0.178 \\
\hline
\end{tabular}

The second term in the upper bound is $-(1-\alpha)(1-\beta)=-0.42625$. For the third term in the upper bound not involving $\gamma(\cdot)$, we get the first part (maximum)

$$
\max _{x \leq l_{0}^{0}}\left(\psi\left(l_{A_{1}}^{1}-x, A_{1}+d\right)-\psi\left(l_{A_{2}}^{1}-x, A_{1}\right)\right)=\max _{x \leq 68.54}(\psi(50-x, 1.29)-\psi(69.08-x, 0.29))
$$

from the table below:

Table 7.3

\begin{tabular}{||c|cccccccc||}
\hline$x$ & 0 & 10 & 20 & 30 & 40 & 50 & 60 & 68.54 \\
\hline$\psi(50-x, 1.29)$ & 0.621 & 0.681 & 0.746 & 0.819 & 0.892 & 0.973 & 1.000 & 1.000 \\
\hline$\psi(69.08-x, 0.29)$ & 0.114 & 0.131 & 0.155 & 0.189 & 0.240 & 0.322 & 0.470 & 0.760 \\
\hline difference & 0.507 & 0.549 & 0.591 & 0.630 & 0.652 & 0.650 & 0.529 & 0.240 \\
\hline
\end{tabular}

The minimum term within the last term

$$
-\min _{x \leq l_{A_{0}}^{0}}\left(\psi\left(l_{A_{1}}^{0}-x, t\right)-\psi\left(l_{A_{2}}^{1}-x, t\right)\right)=-\min _{x \leq 68.54}(\psi(68.54-x, t)-\psi(69.08-x, t))
$$

is achieved for $x=68.54$, and hence equals

$$
-(\psi(0,0.4)-\psi(0.54,0.4))=(\bar{\psi}(0,0.4)-\bar{\psi}(0.54,0.4))=0.149-0.179=-0.03 .
$$

Thus the upper bound for $\Delta(0.40)$, without using the $\gamma(\cdot)$ expression turns out to be

$$
0.138-0.426+0.652-0.03 \approx 0.334
$$

To obtain the upper bound for $\Delta(0.4)$ based on $\gamma($.$) , we first estimate the \gamma(0.4)$ as the minimum of the ratios from the computation in the following table: 
Table 7.4

\begin{tabular}{||c||c|c|c||c||}
\hline$x$ & $\psi\left(l_{A_{2}}^{1}-x, A_{1}-A_{0}\right)$ & $\psi\left(l_{A_{1}}^{0}-x, 0.4-A_{0}\right)$ & $\psi\left(l_{A_{1}}^{1}-x, A_{1}-A_{0}\right)$ & ratio \\
\hline 0 & 0.11 & 0.16 & 0.15 & 1.80 \\
\hline 10 & 0.13 & 0.19 & 0.19 & 1.72 \\
\hline 20 & 0.16 & 0.22 & 0.23 & 1.60 \\
\hline 30 & 0.18 & 0.27 & 0.31 & 1.41 \\
\hline 40 & 0.24 & 0.33 & 0.45 & 1.26 \\
\hline 50 & 0.32 & 0.43 & 0.80 & 0.94 \\
\hline 60 & 0.47 & 0.59 & 1.00 & 1.06 \\
\hline 68.54 & 0.80 & 0.85 & 1.00 & 1.66 \\
\hline
\end{tabular}

This gives:

$$
\begin{gathered}
-(1-\beta) \gamma(0.4) \times P\left(B_{0}\right)+\left[\psi\left(l_{A_{2}}^{1}-l_{A_{0}}^{0}, 0.4-A_{0}\right)+\psi\left(l_{A_{1}}^{1}-l_{A_{0}}^{0}, A_{1}-A_{0}+d\right)\right] \times P\left(B_{j}\right) \\
-0.775 \times 1.312+\psi(69.08-68.54,0.4)+\psi(68.54-50,1.29)=1.09,
\end{gathered}
$$

leading to an eventual upper bound of 0.802 , which is higher than the other method and hence is not considered.

These upper bounds for $\Delta(t)$ for $t=0.4,0.75,1.00,1.37$, as examples over $t$ varying between different alarm times are listed in the table below. Indeed, the difference between upper bounds for two times belonging to the same pair of alarm times are mostly nominal.

Table 7.5

\begin{tabular}{|c|c|c|c|c|c|c|c|c|c|c|c|}
\hline $\mathrm{t}$ & $\mathrm{i}$ & Term 1 & Term 2 & \multicolumn{3}{|c|}{ Term $3(\mathrm{w} / \mathrm{o} \gamma)$} & UB w/o $\gamma$ & term 3 with $\gamma$ & UB with $\gamma$ & best UB \\
\hline & & & & $\mathrm{j}=0$ & $\mathrm{j}=1$ & $\mathrm{j}=2$ & $\mathrm{j}=3$ & & & & \\
\hline 0.4 & 2 & 0.138 & -0.426 & 0.652 & & & & 0.364 & 1.09 & 0.802 & 0.38 \\
\hline 0.75 & 3 & 0.118 & -0.787 & 0.652 & 0.516 & & & 0.498 & 1.151 & 0.481 & 0.48 \\
\hline 1 & 4 & 0.061 & -1.087 & 0.652 & 0.516 & 0.365 & & 0.506 & 1.328 & 0.301 & 0.30 \\
\hline 1.37 & 5 & 0.000 & -1.329 & 0.652 & 0.516 & 0.365 & 0.255 & 0.459 & 2.805 & 1.476 & 0.46 \\
\hline
\end{tabular}

Details for lower bound: While the lower bound is of less significance, we also illustrate the computation of the lower bound for a $t \in\left(A_{1}, A_{2}\right]$ or $i=2$; in particular at $t=0.4$. The first term, based on Table x. 1 turns out to be $0.847 \times 0.01=0.008$. The first term, following slight rearrangement from proposition becomes:

$$
\begin{gathered}
-\alpha \times(1-\beta)+\left\{\beta-\psi\left(0, A_{1}\right)\right\} \times \bar{\psi}(0, t-0.29)+\bar{\psi}\left(u_{0}, t\right)-\psi\left(u_{0}, A_{1}+d\right) \\
=-0.45 \times 0.775+\{0.225-\psi(0,0.29)\} \times \bar{\psi}(0, t-0.29)+\bar{\psi}(50, t)-\psi(50,1.29) \\
=-0.34875+(0.225-0.7996) \times 0.3919+0.786-0.621=-0.409 .
\end{gathered}
$$

Thus, we arrive at a non-trivial lower bound of -0.401 .

$\triangleright$ Application in the case of ultimate ruin probability.

This case can be directly deduced from the previous propositions taking $t \rightarrow \infty$ which implies to consider also $i=k+1$. All the bounds given from (55) to (69) and from (71) to (77) can be rewritten under this hypothesis, in particular we have 
Proposition 5 The difference of ultimate ruin probabilities $\Delta=P\left[T^{M_{r}}=\infty\right]-P\left[T^{M^{k}}=\infty\right]$ entails the following bounds:

$$
\begin{gathered}
\Delta \leq-(1-\alpha)(1-\beta) \sum_{j=0}^{k-1} P\left(B_{j}\right)+\min \left\{-\gamma(1-\beta) \sum_{j=0}^{k-1} P\left(B_{j}\right)+\right. \\
\sum_{j=0}^{k-1}\left[\psi\left(l_{A_{j+2}}^{j+1}-l_{A_{j}}^{j}\right)+\psi\left(l_{A_{j+1}}^{j+1}-l_{A_{j}}^{j}, A_{j+1}-A_{j}+d\right)\right] P\left(B_{j}\right) ; \\
\sum_{j=0}^{k-1}\left[\max _{x \leq l_{A_{j}}^{j}}\left(\psi\left(l_{A_{j+1}}^{j+1}-x, A_{j+1}-A_{j}+d\right)-\psi\left(l_{A_{j+2}}^{j+1}-x, A_{j+1}-A_{j}\right)\right)\right. \\
\left.\left.\quad-\min _{x \leq l_{A_{j}}^{j}}\left(\psi\left(l_{A_{j+1}}^{j}-x\right)-\psi\left(l_{A_{j+2}}^{j+1}-x\right)\right)\right] P\left(B_{j}\right)\right\}
\end{gathered}
$$

where $\gamma=\min _{0 \leq j \leq k-1} \min _{x \leq l_{A_{j}}^{j}} \frac{\psi\left(l_{A_{j+2}}^{j+1}-x, A_{j+1}-A_{j}\right)+\psi\left(l_{A_{j+1}}^{j}-x\right)}{\psi\left(l_{A_{j+1}}^{j+1}-x, A_{j+1}-A_{j}\right)}$

and

$$
\Delta \geq \sum_{j=0}^{k-1} P\left(B_{j}\right)\left[-\alpha+\beta(\alpha+\bar{\psi}(0))-\bar{\psi}(0) \psi\left(0, A_{j+1}-A_{j}\right)+\bar{\psi}\left(u_{j}\right)-\psi\left(u_{j}, A_{j+1}-A_{j}+d\right)\right] .
$$

Remark: Note that if the NPC is violated $(\theta \geq 0)$, as in our numerical examples, then $\bar{\psi}(u)=0$, $\forall u$, which implies that $\Delta=0$. Moreover, as for Proposition 4 , the approximation $P\left(B_{j}\right) \sim(1-\beta)^{j}$ can be used to simplify computations.

Corollary 2 The following approximations can be deduced.

- As $\beta \rightarrow 0$ and for finite $k$ with $k \beta<1$, we have

$$
\begin{aligned}
\Delta & \leq k(\alpha+\min \{0 ; 1-\gamma+(1-\alpha+\gamma) \beta\}) \\
\text { and } \Delta \geq & k\left(-\alpha+\bar{\psi}(0) \bar{\psi}\left(0, \delta_{k+1}^{A}\right)-\psi\left(0, \delta_{k+1}^{A}+d\right)\right) \\
\text { with } & \delta_{k+1}^{A}=\max _{0 \leq j \leq k-1}\left(A_{j+1}-A_{j}\right) .
\end{aligned}
$$

- As $k \rightarrow \infty$, we have

$$
\begin{aligned}
\Delta \leq \frac{1}{\beta}\left[\max _{j \geq 0} \max _{x \leq l_{A_{j}}^{j}}\left(\psi\left(l_{A_{j+1}}^{j+1}-x, A_{j+1}-A_{j}\right)-\psi\left(l_{A_{j+2}}^{j+1}-x, A_{j+1}-A_{j}\right)\right)\right. \\
\left.-\min _{j \geq 0} \min _{x \leq l_{A_{j}}^{j}}\left(\psi\left(l_{A_{j+1}}^{j}-x\right)-\psi\left(l_{A_{j+2}}^{j+1}-x\right)\right)-(1-\alpha)(1-\beta)\right] \\
\text { and } \Delta \geq \alpha+\bar{\psi}(0)-\frac{\alpha-\bar{\psi}(0) \bar{\psi}\left(0, \delta_{k+1}^{A}\right)+\psi\left(0, \delta_{k+1}^{A}+d\right)}{\beta} .
\end{aligned}
$$

Note that we chose to present approximated bounds easy to compute, although rougher than the ones that could also be deduced from Proposition 5. 


\subsubsection{Direct method}

An alternative way to compare the two ultimate ruin probabilities may be the following.

\section{- Comparison of finite time ruin probabilities.}

We will evaluate

$$
\Delta(t)=P\left[T^{M_{r}}>t\right]-P\left[T^{M^{k}}>t\right]
$$

for $t>A_{k}$; it might be considered as the main case of interest since the risk of ruin before $A_{k}$ is small enough for the model $M^{k}$ to define $k$ alarms.

We have $P\left[T^{M_{r}}>t\right]=\bar{\psi}\left(\sum_{n=0}^{k} u_{n} e^{-r A_{n}}, t\right)$ and, using (62),

$$
\begin{aligned}
P\left[T^{M^{k}}>t\right] & =P\left[B_{k} \cap\left(T\left(A_{k}, \sum_{n=0}^{k} u_{n}\right)>t\right)\right]=P\left(B_{k}\right) \bar{\psi}_{A_{k}}\left(l_{A_{k+1}}^{k+1}, t \mid B_{k}\right) \\
& =P\left(B_{k}\right) \int_{-\infty}^{l_{A_{k+1}+1}^{k+1}} \bar{\psi}\left(l_{A_{k+1}}^{k+1}-x, t-A_{k}\right) P\left[B_{k} \mid R_{A_{k}}=x\right] d F_{R_{A_{k}}}(x)
\end{aligned}
$$

that satisfies

$$
P^{2}\left(B_{k}\right) \bar{\psi}\left(0, t-A_{k}\right) \leq P\left[T^{M^{k}}>t\right] \leq P^{2}\left(B_{k}\right)
$$

hence

$$
\bar{\psi}\left(\sum_{n=0}^{k} u_{n} e^{-r A_{n}}, t\right)-P^{2}\left(B_{k}\right) \leq \Delta(t) \leq \bar{\psi}\left(\sum_{n=0}^{k} u_{n} e^{-r A_{n}}, t\right)-P^{2}\left(B_{k}\right) \bar{\psi}\left(0, t-A_{k}\right) .
$$

Note that the difference between the two above bounds is $P^{2}\left(B_{k}\right) \psi\left(0, t-A_{k}\right)$ and increases with the time $t$. Hence we can conclude that

$$
\max \left(-1 ; \bar{\psi}\left(\sum_{n=0}^{k} u_{n} e^{-r A_{n}}, t\right)-P^{2}\left(B_{k}\right)\right) \leq \Delta(t) \leq \min \left(1 ; \bar{\psi}\left(\sum_{n=0}^{k} u_{n} e^{-r A_{n}}, t\right)-P^{2}\left(B_{k}\right) \bar{\psi}\left(0, t-A_{k}\right)\right) .
$$

When considering the alarm times definition (15) and (61), the lower and upper bound of $\Delta(t)$ given in (78) are equivalent, respectively, to

$$
\bar{\psi}\left(\sum_{n=0}^{k} u_{n} e^{-r A_{n}}, t\right)-(1-\beta)^{2 k} \simeq \bar{\psi}\left(\sum_{n=0}^{k} u_{n} e^{-r A_{n}}, t\right)-e^{-2 \beta k} \underset{2 \beta k<1}{\sim} 2 \beta k-\psi\left(\sum_{n=0}^{k} u_{n} e^{-r A_{n}}, t\right)
$$

and

$$
\begin{gathered}
\bar{\psi}\left(\sum_{n=0}^{k} u_{n} e^{-r A_{n}}, t\right)-(1-\beta)^{2 k} \bar{\psi}\left(0, t-A_{k}\right) \simeq \bar{\psi}\left(\sum_{n=0}^{k} u_{n} e^{-r A_{n}}, t\right)-e^{-2 \beta k} \bar{\psi}\left(0, t-A_{k}\right) \\
\simeq 2 \beta k+\psi\left(0, t-A_{k}\right)(1-2 \beta k)-\psi\left(\sum_{n=0}^{k} u_{n} e^{-r A_{n}}, t\right), \quad \text { for } 2 \beta k<1 .
\end{gathered}
$$

\section{- Comparison of ultimate ruin probabilities:}


Since we set $A_{k+1}=+\infty$, we can write

$$
\Delta=P\left[T^{M_{r}}=\infty\right]-P\left[T^{M^{k}}=\infty\right]=\bar{\psi}\left(\sum_{i=0}^{k} u_{i} e^{-r A_{i}}\right)-P\left(B_{k+1}\right) .
$$

When considering our specific alarm time, it comes, using (61),

$$
\Delta \simeq 1-\psi\left(\sum_{i=0}^{k} u_{i} e^{-r A_{i}}\right)-(1-\beta)^{k+1}
$$

Whenever $\beta(k+1)<1$, we have

$$
\Delta \simeq \beta(k+1)-\psi\left(\sum_{i=0}^{k} u_{i} e^{-r A_{i}}\right)
$$

whereas, for $k \rightarrow \infty$,

$$
\Delta \simeq 1-\psi\left(\sum_{i=0}^{\infty} u_{i} e^{-r A_{i}}\right)
$$

Note that if we assume that $u=\sum_{i=0}^{k} u_{i} e^{-r A_{i}} \rightarrow \infty$, then we can consider for the classical model under NPC the Cramér's bound $\psi(u) \sim K e^{-C u}$, where $C$ is the adjustment coefficient.

\subsection{Comparison of systems with or without an alarm: Numerical illustration}

In this section we attempt to empirically verify if and/or when it is advantageous to have an alarm system with additional amounts being added at the sound of alarm as opposed to starting with higher initial capital (and no subsequent addition to the capital). To draw a fair comparison, it is imperative not only to subject both the risk processes to identical claim process but also ensure that the additional amounts provided for the non-alarm system is in congruence with the amounts added subsequently to the alarm-system. Since in our main approach, alarm times are non-random (parameters or fixed values), it is straightforward to consider the discounted amount as per the rate of interest $r$. However even if the alarm times are to be random variables (as in the other approaches indicated in this work), it would be fair to work with the expected discounted amount.

While comparing performances in the simulation set up, one needs to be careful about the choice of rate of interest $r$, specially because in the framework we have kept the unit of the time frame unspecified. Consequently, we take several wide-ranging values of $r$ which at first glance may appear to be unreasonable. In particular, we took the rate of interests to be $0 \%, 5 \%, 10 \%, 20 \%$, $30 \%, 50 \%, 75 \%, 100 \%, 150 \%, 200 \% 300 \%, 500 \%$ and $\infty$ (table represents part of the result). The comparison is carried out in the framework of Example 2, where the alarm system starts with an initial capital of $u=50$ and additional 10\% (=5) of the initial capital being added to the system at each of the alarm times. In the framework, we allowed for as many alarms as required and alarm goes off for only on four occasions, viz. at $t=0.29,0.58,0.91$ and 1.28 with $\alpha=0.45, \beta=0.225$ and $d=1$. Some of the key survival probabilities $P[T \geq t]$ are reported in Table 8 . For more complete comparison, the probabilities of survivals up to different time points [viz. survival function of the corresponding ruin times] of the systems are shown in Figure 8.

This table and figure, clearly brings out the advantages of the alarm system. For example, if one considers the survival at time $=3$ to be the reference frame, then it is beneficial to have the alarm system as opposed to having additional capital in the beginning as long as the rate of interest is $10 \%$ or higher. Of course, if one considers a very short time window, the conclusion would be otherwise. 
Figure 8: Survival function with or without alarm (different ROI)

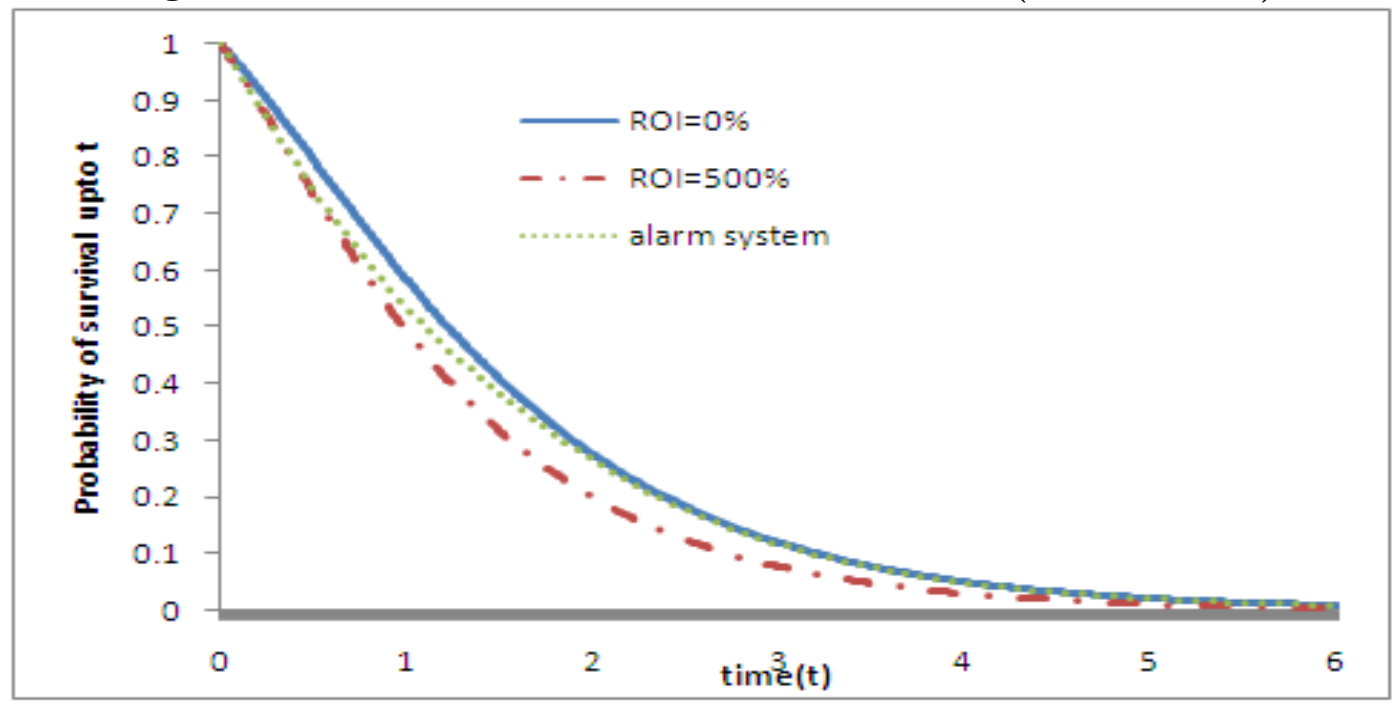

Table 8: Comparing Survival Probabilities between Systems without \& with alarm

\begin{tabular}{|c|c|c|c|c|c|c|c|c|c|c|c|}
\hline \multirow{4}{*}{$\mathrm{t}$} & \multicolumn{11}{|c|}{ NO alarm system with additional equivalent initial capital } \\
\hline & \multirow[b]{3}{*}{$P\left[T^{M^{A}} \geq t\right)$} & $0 \%$ & $10 \%$ & $30 \%$ & $50 \%$ & $100 \%$ & $150 \%$ & $200 \%$ & $500 \%$ & $\infty$ & \\
\hline & & 70.000 & 68.540 & 65.996 & 63.875 & 59.944 & 57.341 & 55.564 & 51.509 & 50.000 & $u_{0}\left(M_{r}\right)$ \\
\hline & & \multicolumn{10}{|c|}{$P\left[T^{M_{r}} \geq t\right]$} \\
\hline 0.01 & 0.995 & 0.996 & 0.996 & 0.996 & 0.996 & 0.996 & 0.996 & 0.996 & 0.995 & 0.995 & \\
\hline 0.1 & 0.950 & 0.964 & 0.963 & 0.962 & 0.961 & 0.958 & 0.956 & 0.955 & 0.952 & 0.950 & \\
\hline 0.2 & 0.896 & 0.924 & 0.922 & 0.919 & 0.917 & 0.911 & 0.908 & 0.905 & 0.899 & 0.896 & \\
\hline 0.3 & 0.842 & 0.883 & 0.880 & 0.876 & 0.872 & 0.865 & 0.859 & 0.855 & 0.845 & 0.841 & \\
\hline 0.4 & 0.794 & 0.840 & 0.837 & 0.832 & 0.826 & 0.816 & 0.809 & 0.804 & 0.791 & 0.785 & \\
\hline 0.5 & 0.743 & 0.796 & 0.792 & 0.785 & 0.779 & 0.767 & 0.758 & 0.751 & 0.736 & 0.729 & \\
\hline 0.75 & 0.632 & 0.687 & 0.682 & 0.673 & 0.664 & 0.648 & 0.636 & 0.627 & 0.607 & 0.599 & \\
\hline 1 & 0.536 & 0.584 & 0.578 & 0.568 & 0.558 & 0.540 & 0.527 & 0.517 & 0.494 & 0.486 & \\
\hline 1.25 & 0.451 & 0.490 & 0.484 & 0.472 & 0.462 & 0.443 & 0.430 & 0.420 & 0.397 & 0.388 & \\
\hline 1.5 & 0.384 & 0.409 & 0.403 & 0.391 & 0.380 & 0.361 & 0.349 & 0.339 & 0.318 & 0.310 & \\
\hline 1.75 & 0.321 & 0.337 & 0.331 & 0.320 & 0.311 & 0.293 & 0.281 & 0.272 & 0.253 & 0.246 & \\
\hline 2 & 0.265 & 0.276 & 0.270 & 0.260 & 0.252 & 0.235 & 0.225 & 0.217 & 0.200 & 0.194 & \\
\hline 2.5 & 0.177 & 0.183 & 0.178 & 0.171 & 0.164 & 0.151 & 0.143 & 0.137 & 0.125 & 0.120 & \\
\hline 3 & 0.116 & 0.119 & 0.116 & 0.110 & 0.105 & 0.096 & 0.090 & 0.086 & 0.078 & 0.074 & \\
\hline 3.5 & 0.076 & 0.077 & 0.075 & 0.070 & 0.067 & 0.060 & 0.056 & 0.053 & 0.048 & 0.045 & \\
\hline 4 & 0.049 & 0.050 & 0.048 & 0.045 & 0.042 & 0.038 & 0.035 & 0.033 & 0.029 & 0.028 & \\
\hline 5 & 0.021 & 0.021 & 0.020 & 0.019 & 0.018 & 0.016 & 0.015 & 0.014 & 0.012 & 0.011 & \\
\hline 6 & 0.009 & 0.009 & 0.008 & 0.008 & 0.007 & 0.006 & 0.006 & 0.005 & 0.005 & 0.005 & \\
\hline 7 & 0.004 & 0.004 & 0.004 & 0.003 & 0.003 & 0.003 & 0.003 & 0.002 & 0.002 & 0.002 & \\
\hline
\end{tabular}

Example 2 setup: claims occur as per Poisson process with $\lambda=20$ with claim severity following Pareto distribution with $\rho=1$ and $\kappa=0.95$. Premium function is linear $p_{t}=40 t$.

Alarm system has initial capital $u_{0}=50$, with addition $10 \%$ of it $\left(u_{i}=5, \forall i\right)$ being added at each alarm. For the given set of parameters, the 4 alarm times $A_{i}$ 's are found to be $0.29,0.58,0.91$, and 1.28 and subsequently there are no more alarms.

In the corresponding No-alarm systems $M_{r}, r$ represents the interest rate determining equivalent initial capital

$$
u_{0}\left(M_{r}\right)=u_{0}+\sum_{i} e^{-r A_{i}} u_{i}
$$

To see another illustration, we revisit Example 1, where the severity has an exponential distribution. Some of the key survival probabilities $P[T \geq t]$ are reported in Table 9 . For more complete comparison, the probabilities of survivals up to different time points [viz. survival function of the corresponding ruin times] of the systems are shown in Figure 9. 
Figure 9: Survival function with or without alarm (different ROI): Example 1

Survival probability with alarm vs without alarm

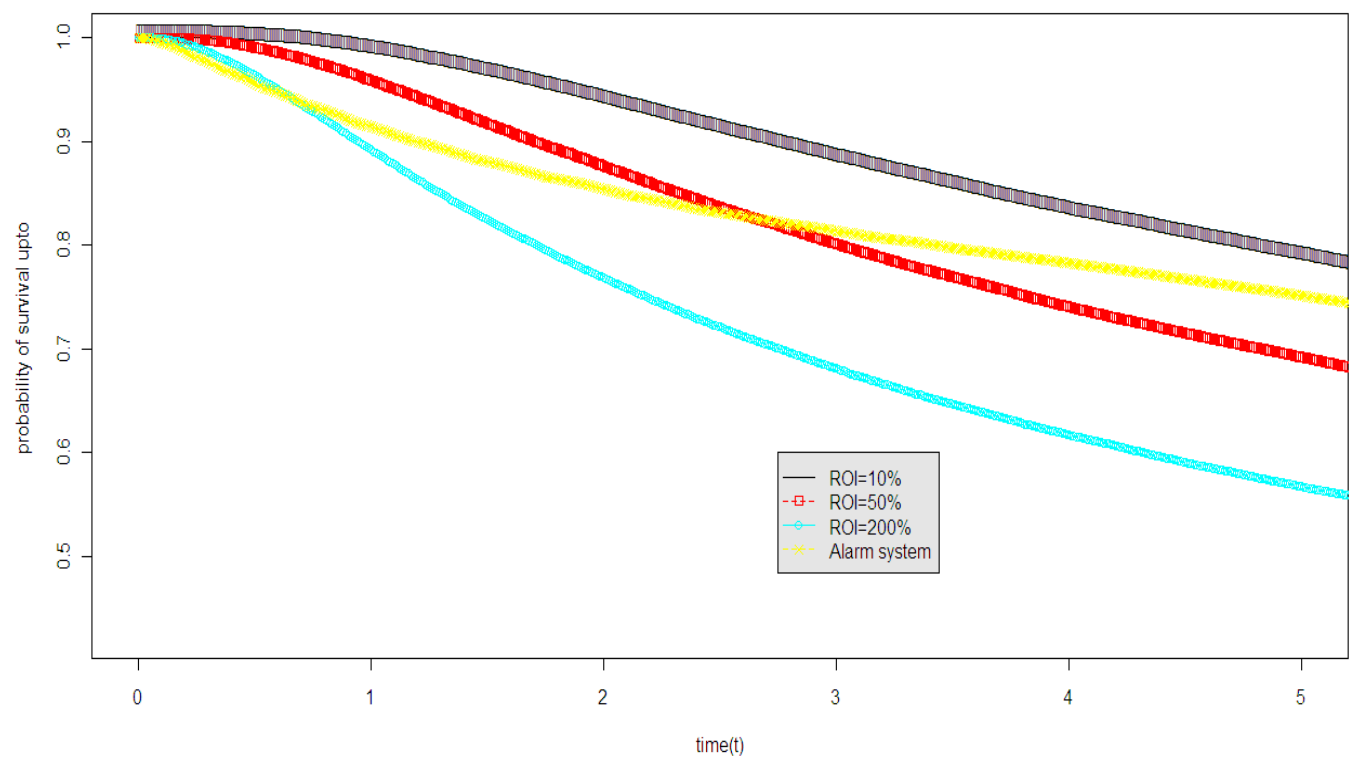

Table 9: Comparing Survival Probabilities between Systems without \& with alarm: Exponential Severity

\begin{tabular}{|c|c|c|c|c|c|c|c|c|c|c|c|}
\hline \multirow{4}{*}{$\mathrm{t}$} & \multicolumn{11}{|c|}{ NO alarm system with additional equivalent initial capital } \\
\hline & \multirow[b]{3}{*}{$P\left[T^{M^{A}} \geq t\right)$} & $0 \%$ & $10 \%$ & $20 \%$ & $30 \%$ & $50 \%$ & $75 \%$ & $100 \%$ & $500 \%$ & $\infty$ & $r$ \\
\hline & & 37.5000 & 34.6412 & 32.3490 & 30.4933 & 27.7234 & 25.4101 & 23.8539 & 18.2586 & 15.0000 & $u_{0}\left(M_{r}\right)$ \\
\hline & & \multicolumn{10}{|c|}{$P\left[T^{M_{r}} \geq t\right]$} \\
\hline 0.25 & 0.981 & 1.000 & 1.000 & 1.000 & 0.999 & 0.999 & 0.997 & 0.996 & 0.983 & 0.962 & \\
\hline 0.5 & 0.957 & 0.999 & 0.998 & 0.997 & 0.995 & 0.991 & 0.985 & 0.980 & 0.942 & 0.898 & \\
\hline 0.75 & 0.933 & 0.996 & 0.993 & 0.990 & 0.986 & 0.977 & 0.966 & 0.956 & 0.896 & 0.837 & \\
\hline 1 & 0.914 & 0.991 & 0.986 & 0.980 & 0.973 & 0.958 & 0.942 & 0.928 & 0.852 & 0.783 & \\
\hline 1.25 & 0.896 & 0.984 & 0.976 & 0.967 & 0.957 & 0.938 & 0.916 & 0.899 & 0.812 & 0.737 & \\
\hline 1.5 & 0.880 & 0.975 & 0.964 & 0.952 & 0.939 & 0.916 & 0.891 & 0.872 & 0.777 & 0.699 & \\
\hline 1.75 & 0.865 & 0.965 & 0.951 & 0.936 & 0.922 & 0.895 & 0.867 & 0.846 & 0.745 & 0.667 & \\
\hline 2 & 0.853 & 0.954 & 0.937 & 0.921 & 0.904 & 0.874 & 0.844 & 0.822 & 0.717 & 0.637 & \\
\hline 2.5 & 0.831 & 0.931 & 0.910 & 0.890 & 0.870 & 0.836 & 0.803 & 0.778 & 0.669 & 0.588 & \\
\hline 3 & 0.814 & 0.909 & 0.883 & 0.860 & 0.838 & 0.801 & 0.765 & 0.739 & 0.628 & 0.548 & \\
\hline 3.5 & 0.798 & 0.885 & 0.857 & 0.831 & 0.808 & 0.769 & 0.732 & 0.705 & 0.594 & 0.516 & \\
\hline 4 & 0.784 & 0.862 & 0.833 & 0.806 & 0.781 & 0.741 & 0.703 & 0.675 & 0.565 & 0.489 & \\
\hline 5 & 0.752 & 0.820 & 0.788 & 0.758 & 0.732 & 0.691 & 0.652 & 0.624 & 0.516 & 0.444 & \\
\hline 6 & 0.720 & 0.782 & 0.748 & 0.717 & 0.690 & 0.649 & 0.611 & 0.583 & 0.479 & 0.411 & \\
\hline 7 & 0.692 & 0.748 & 0.714 & 0.682 & 0.655 & 0.614 & 0.576 & 0.549 & 0.448 & 0.383 & \\
\hline 8 & 0.666 & 0.719 & 0.684 & 0.652 & 0.625 & 0.584 & 0.548 & 0.521 & 0.423 & 0.361 & \\
\hline 9 & 0.642 & 0.692 & 0.656 & 0.625 & 0.599 & 0.559 & 0.522 & 0.496 & 0.401 & 0.342 & \\
\hline 10 & 0.620 & 0.667 & 0.632 & 0.601 & 0.575 & 0.535 & 0.500 & 0.475 & 0.383 & 0.326 & \\
\hline 15 & 0.537 & 0.574 & 0.541 & 0.512 & 0.488 & 0.452 & 0.420 & 0.398 & 0.318 & 0.269 & \\
\hline 20 & 0.477 & 0.509 & 0.478 & 0.451 & 0.429 & 0.396 & 0.368 & 0.349 & 0.278 & 0.234 & \\
\hline 25 & 0.434 & 0.463 & 0.433 & 0.408 & 0.388 & 0.357 & 0.331 & 0.313 & 0.249 & 0.209 & \\
\hline 30 & 0.402 & 0.427 & 0.400 & 0.376 & 0.357 & 0.329 & 0.305 & 0.288 & 0.228 & 0.192 & \\
\hline 35 & 0.375 & 0.399 & 0.373 & 0.350 & 0.332 & 0.305 & 0.283 & 0.267 & 0.211 & 0.178 & \\
\hline 40 & 0.354 & 0.376 & 0.350 & 0.328 & 0.312 & 0.286 & 0.265 & 0.250 & 0.198 & 0.166 & \\
\hline 45 & 0.335 & 0.356 & 0.332 & 0.311 & 0.295 & 0.271 & 0.251 & 0.236 & 0.187 & 0.157 & \\
\hline 50 & 0.320 & 0.340 & 0.316 & 0.296 & 0.281 & 0.258 & 0.239 & 0.225 & 0.178 & 0.150 & \\
\hline
\end{tabular}

Example 1 setup: claims occur as per Poisson process with $\lambda=20$ with claim severity following exponential distribution with $\rho=0.5$ Premium function is linear $p_{t}=40 t$.

Alarm system has initial capital $u_{0}=15$, with addition $10 \%$ of it $\left(u_{i}=1.5, \forall i\right)$ being added at each alarm. For the given set of parameters, the 15 alarm times $A_{i}$ 's are found to be $0,0.105,0.23,0.37,0.54,0.725,0.93,1.185,1.46,1.75,2.105,2.43$, $2.785,3.14$ and 3.55 ; subsequently there are no more alarms. 


\section{Alternative Approaches for Defining Alarms, Extensions and Concluding comments}

\subsection{Severity based Alarm}

A simple and naive approach to set up a system alarm evolves by working directly with the severity of claims and detecting very large claims which might lead to ruin. This approach might work for small claim sizes, since we know that, in the case of claim sizes heavily distributed, ruin might occur only as the result of one large claim size (see e.g. [16]).

One classic way to do so, when considering the C-L model with i.i.d. claims, is to look for $\gamma=\gamma(t)$ such that

$$
\mathbb{E}\left[\sum_{i=1}^{N(t)} 1_{\left(X_{i}>\gamma\right)}\right]=1
$$

where $\gamma$ represents the return level of order $t$ that is expected to be exceeded in one out of every $t$-period.

Since $\left(N_{t}\right)$ is supposed to be independent of the $X_{i}$ 's, we can write

$$
\mathbb{E}\left[\sum_{i=1}^{N(t)} 1_{\left(X_{i}>\gamma\right)}\right]=\mathbb{E}\left(\mathbb{E}\left[\sum_{i=1}^{N(t)} 1_{\left(X_{i}>\gamma\right)} \mid N_{t}\right]\right)=\mathbb{E}[N(t)] P\left[X_{i}>\gamma\right]=\lambda t \bar{F}(\gamma) .
$$

Hence (79) is equivalent to

$$
F(\gamma)=1-\frac{1}{\lambda t}
$$

Therefore estimating the return level $\gamma$ of order $t$ corresponds to estimate a high quantile of order $p_{t}=1-\frac{1}{\lambda t}$.

When $F$ is continuous, tools of the Extreme Value Theory (EVT) can be used to express the estimators of the return level and period as simple functionals of the estimated parameters either of the GEV distribution (defined for all $x>\mu-\sigma / \xi, \xi, \mu \in \mathbb{R}$ and $\sigma>0$, by $H_{\xi}(x)=e^{-(1+\xi(x-\mu) / \sigma)^{-1 / \xi}}$ ), or of the GPD

$$
G_{(\xi, \sigma(u))}=\left\{\begin{array}{ll}
1-(1+\xi y / \sigma(u))^{-1 / \xi} & \text { if } \xi \neq 0 \\
1-e^{-y / \sigma(u)} & \text { if } \xi=0
\end{array},\right.
$$

for $0 \leq y \leq-\sigma(u) / \xi$ if $\xi<0$ and $\forall y \geq 0$ otherwise (where $\sigma($.$) is a positive function), when using$ the POT method to approximate the distribution of the $N_{u}$ observations above a given level $u$ among $n$ observations (for more details on these methods, we refer the reader e.g. to [6]).

The estimations of the extreme quantile of order $p_{t}$ and of the associated return period are, respectively, given by

$$
\hat{\gamma}_{p_{t}}^{G E V}=\left\{\begin{array}{ll}
\hat{\mu}-\hat{\sigma}\left(1-\left(-\log \left(p_{t}\right)\right)^{-\hat{\xi}}\right) & \text { if } \xi \neq 0 \\
\hat{\mu}-\hat{\sigma} \log \left(-\log \left(p_{t}\right)\right) & \text { if } \xi=0
\end{array} \quad \& \quad \hat{t}^{G E V}=\frac{1}{1-H_{\hat{\xi}}\left(\gamma_{\hat{p}_{t}}\right)}\right.
$$

or

$$
\hat{\gamma}_{p_{t}}^{G P D}=u+\frac{\hat{\sigma}}{\hat{\xi}}\left(\left(n\left(1-p_{t}\right) / N_{u}\right)^{-\hat{\xi}}-1\right), \forall \xi \neq 0 \quad \& \quad \hat{t}^{G P D}=\frac{n}{N_{u}} \cdot \frac{1}{1-G_{\hat{\xi}, \hat{\sigma}}\left(\gamma_{\hat{p}_{t}}\right)},
$$

with $N_{u}=\#\left\{j: 1 \leqslant j \leqslant n, X_{j}>u\right\}$. 
If $F$ is discrete, we propose an approximation of the return level and period. Two ways are possible. The first one consists in using an estimate of the upper bound of the return level $\gamma$ (satisfying (4.1)) proposed in [8] (that applies to any d.f. $F$ and any $t$ ), namely

$$
\hat{\gamma}_{t} \leq \inf \left\{\hat{b}_{t}(\alpha, \beta)=\left(\frac{\lambda t}{\left(1-\frac{1}{\lambda t}\right)^{\beta}} \frac{1}{n} \sum_{i=1}^{n}\left(\frac{i}{n}\right)^{\beta} x_{i, n}^{\alpha}\right)^{\frac{1}{\alpha}} ; \alpha \in\left(\varepsilon, \alpha^{*}\right], \beta \in\left(\varepsilon, \beta^{*}\right]\right\}
$$

for some constants $\alpha^{*}$ and $\beta^{*}$ (depending on the data set), $\varepsilon$ close to 0 , and where $x_{i, n}$ denotes the $i$ th largest observation of the data set $\left(x_{i}, 1 \leq i \leq n\right)$.

The second way consists in smoothing the discrete data (e.g. via a kernel estimation of the spectral density) to generate a continuous sample in order to be able to apply EVT tools, as described above.

These two approaches have been settled and applied in various contexts in [4], in particular in epidemiology (see [7] when using the first approach).

For both approaches, we proceed in two steps to estimate the return level associated to a new observation. First we estimate the high quantile $\gamma_{t}$ of order $p_{t}$ or consider its upper bound $\hat{b}_{t}$ when choosing the first approach, and give the plot $\left(t, \hat{\gamma}_{t}\right)$ or $\left(t, \hat{b}_{t}\right)$ for all $t$. Then, if $x_{t_{0}}$ denotes the new observation at time $t_{0}$, then we set $x_{t_{0}}=\hat{\gamma}_{T}$ or $\hat{b}_{T}$ and read the corresponding return period value $T$. Now, looking at the observations, if there already exists a higher value than $\hat{\gamma}_{T}$ or $\hat{b}_{T}$ on the interval $\left(t_{0}-T, t_{0}\right)$, then we consider $t_{0}$ as an alarm time, since by definition a return level $z_{T}$ associated with a given return period $T$ corresponds to the level expected to be exceeded on average only once every $T$ time units. Note that a return period is usually used in a predictive way; here we used it when looking backward, since we can write for any $0 \leq s<t$ (and setting $X_{0}=0$ ),

$$
\mathbb{E}\left(\sum_{i=0}^{N(t)} 1_{\left(X_{i}>\gamma\right)}\right)=\mathbb{E}\left(\sum_{i=N(s)}^{N(s+t)} 1_{\left(X_{i}>\gamma\right)}\right)
$$

the claims being i.i.d., $\left(N_{t}\right)$ being a Poisson process and independent of the $X_{i}$ 's.

We refer the reader to [4] where this method has been applied considering a simulated sample of severity claims having a log-discrete distribution $F$ with parameter $\alpha=0.9$, mean $\mu=$ $\frac{-\alpha}{(1-\alpha) \log (1-\alpha)} \simeq 3.9$, and when choosing e.g. $c=4$ to satisfy the NPC and the intensity of the Poisson process $\left(N_{t}\right)$ to be $\lambda=1$.

Remark: To refine this method, one way would be to work on the $Z_{i}$ 's rather than on the $X_{i}$ 's, the relation between very large values of $Z_{i}$ and ruin being more explicit. The price to be paid is that, although the $Z_{i}$ 's are i.i.d., they are not independent of the Poisson process $\left(N_{t}\right)$, hence the computation of $\mathbb{E}\left(\sum_{i=1}^{N(t)} 1_{\left(X_{i}>\gamma\right)}\right)$ does not lead to an explicit expression for $\gamma$. Indeed, we have

$$
\mathbb{E}\left(\sum_{i=1}^{N(t)} 1_{\left(Z_{i}>\gamma\right)}\right)=\sum_{n \geq 1} \mathbb{E}\left(\sum_{i=1}^{N(t)} 1_{\left(Z_{i}>\gamma\right)} \mid N(t)=n\right) P[N(t)=n]=\sum_{n \geq 1} \sum_{i=1}^{n} p_{i, n}
$$

where

$$
p_{i, n}=P\left[Z_{i}>\gamma, N(t)=n\right]=P\left[X_{i}-c W_{i}>\gamma, \sum_{j=1}^{n} W_{j} \leq t<\sum_{j=1}^{n+1} W_{j}\right] .
$$


Then, conditioning given $W_{i}$, if $f_{W_{1}}$ denotes the density function of $W_{i}$, we obtain

$$
\begin{aligned}
\mathbb{E}\left(\sum_{i=1}^{N(t)} 1_{\left(Z_{i}>\gamma\right)}\right) & =\sum_{n \geq 1} \sum_{i=1}^{n} \int_{\mathbb{R}} P\left(X_{i}-c x>\gamma, \sum_{j=1, j \neq i}^{n} W_{j} \leq t-x<\sum_{j=1, j \neq i}^{n+1} W_{j}\right) f_{W_{1}}(x) d x \\
& =\sum_{n \geq 1} \sum_{i=1}^{n} \int_{\mathbb{R}} P\left[X_{i}-c x>\gamma\right] P[N(t-x)=n-1] f_{W_{1}}(x) d x \\
& =\sum_{n \geq 1} \frac{n}{\lambda} \int_{0}^{+\infty} \bar{F}(\gamma+c x) P[N(t-x)=n-1] e^{-\lambda x} d x \\
& =e^{-\lambda t} \sum_{n \geq 1} \frac{n}{(n-1) !} \lambda^{n-2} \int_{0}^{+\infty}(t-x)^{n-1} \bar{F}(\gamma+c x) d x .
\end{aligned}
$$

Therefore we have to find $\gamma_{\text {num solving }}$

$$
e^{-\lambda t} \sum_{n \geq 1} \frac{n}{(n-1) !} \lambda^{n-2} \int_{0}^{+\infty}(t-x)^{n-1} \bar{F}(\gamma+c x) d x=1
$$

that might be done numerically. Then the same method as developed previously on the $X_{i}$ 's applies when considering $\gamma_{\text {num }}$ in order to define the return period associated with each new observation.

Finally, we could also consider the simple C-L model with i.i.d. claims and the severities amount between the times $k-1$ and $k$, represented by $Y=\left(Y_{k} ; k \in \mathbb{N}^{*}\right)$ with $Y_{k}:=\sum_{i=1+N(k-1)}^{N(k)} X_{i}$.

Our goal being to set up a surveillance system for any unusual high severity amount, we would have to estimate the return period associated to such high amount using

$$
\mathbb{E}\left[\sum_{k=1}^{t} 1_{\left(Y_{k}>\gamma\right)}\right]=1
$$

Note that the $Y_{k}$ 's are i.i.d. with d.f. $F_{Y}$ given by

$$
F_{Y}(x)=\sum_{m=0}^{\infty} P[N(1)=m] P\left[\sum_{i=1}^{m} X_{i} \leq x\right]
$$

If $F_{Y}$ is discrete, some extra work would be required to obtain the convergence of the estimate of the upper bound of the return level, when using the bounds method.

\subsection{Defining Alarm at the first hit of specified level}

In the approach we propose to raise an alarm whenever the risk process reached a suitably low level for the first time. The suitability is defined in terms of having high enough risk of ruin in impending future as in Section 2.

Definition. First we define an alarm level $\gamma>0$ of the risk process that satisfies, for suitable $d>0$ and $\alpha>0$,

$$
\inf _{s>0} P[T(u)<s+d \mid T(u-\gamma)=s] \geq 1-\alpha
$$

where we recall that

$$
T(u-\gamma)=\inf \{s>0: R(s)>u-\gamma\}
$$


Remark: we chose here to consider the continuous notation for the ruin time, so the rough conditioning notation refers to probability density function notation.

It is clear that such a $\gamma$ may not exist for all $d$ and $\alpha$ for all the risk processes. The important question is whether it exists for some reasonable choices of these problem-specifying parameters and for some interesting risk processes; the same concern is valid for any approach.

Definition. A random alarm time $A$ may be defined corresponding to the alarm level $\gamma$, defined in (80), as follows:

$$
A=T(u-\gamma)
$$

More generally, setting $\gamma_{1}=\gamma\left(\right.$ and $\left.u=u_{0}\right)$, we can define a sequence of alarm levels $\left(\gamma_{i}\right)_{i \geq 1}$ where $\gamma_{i+1}$ is the solution in $\gamma$ of

$$
\inf _{s>0} P\left[T\left(A_{i}, \sum_{j=1}^{i} u_{j}\right) \leq s+d \mid T\left(A_{i}, \sum_{j=1}^{i} u_{j}-\gamma\right)=s\right] \geq 1-\alpha,
$$

as well as a sequence of alarm times $\left(A_{i}\right)_{i \geq 1}$ defined by $A_{1}=A$ and, for $i \geq 1$,

$$
A_{i+1}=T\left(A_{i}, \sum_{j=1}^{i} u_{j}-\gamma_{i+1}\right)
$$

As an alternative formulation, the conditional probability in (82) may be replaced with the probability of joint events, like remarked earlier.

Remark. It is also possible to define alarm times through the existence of the smallest level below the accumulated capital that the process $R$ will have necessarily to cross at least once before the ruin occurs subject to surviving up to that alarm time with sufficiently high probability. To formalize, as a variation, we work with probabilities of joint events rather than with conditional probabilities, as given below.

Given $d>0$ and $\alpha>0$, the first alarm time may be defined as:

$$
A=\inf _{\gamma>0}\left\{\inf \left\{s>0: u>R_{s}>u-\gamma \text { and } \psi_{s}(u, s+d) \geq 1-\alpha\right\}\right\} .
$$

$\left(A_{0}:=0\right)$. More generally, the subsequent alarm times $A_{i}, i>1$, may be defined as:

$$
\begin{array}{r}
A_{i+1}=\inf _{\gamma>0} \inf \left\{s>A_{i}: \sum_{j=0}^{i} u_{j}>R_{s}>\sum_{j=0}^{i} u_{j}-\gamma\right. \text { and } \\
\left.\psi_{s}\left(\sum_{j=0}^{i} u_{j}, s+d\right) \geq 1-\alpha\right\} .
\end{array}
$$

Remark: it might be the same to compute the alarm times with a given capital $u$, adding the extra amounts only when resetting the system. 
With the random alarm time $A$ defined in (81), we need to evaluate $P[T(u)>s+d \mid T(u-\gamma)=s]$ : it is related to the distribution of the surplus prior to ruin but at a given time, and might be computed as in [18], or similarly to [5] (see $\S 8.5$, p.165).

The comparison between alarm and no-alarm system can be carried through for this random alarm time as well, using that

$$
P\left[T^{M_{r}}=+\infty\right]-P\left[T^{M^{A}}=+\infty\right]=\int_{0}^{+\infty}\left(P\left[T^{M_{r}}=+\infty\right]-P\left[T^{M^{A}}=+\infty \mid A=t\right]\right) d F_{A}(t),
$$

where $F_{A}$ denotes the c.d.f. of the r.v. $A$ and where the conditional probability appearing in the integral can be computed via Proposition 1.

\subsection{Alarm System for Reinsurance}

To start with, consider 1 cedent and 1 reinsurer model, and denoting their ruin times by $T^{c}$ and $T^{c}$ respectively, the following are some of the options for devising alarm system along one of the approaches developed previously.

$$
\begin{gathered}
A=\inf \left\{s>0: P\left[\min \left(T^{r}, T^{c}\right) \leq s+d \mid \min \left(T^{r}, T^{c}\right)>s\right] \geq 1-\alpha\right\} \\
\quad \text { or } A=\inf \left\{s>0: P\left[T^{c} \leq s+d \mid \min \left(T^{r}, T^{c}\right)>s\right] \geq 1-\alpha\right\} \\
\quad \text { or } A=\inf \left\{s>0: P\left[T^{r} \leq s+d \mid \min \left(T^{r}, T^{c}\right)>s\right] \geq 1-\alpha\right\}
\end{gathered}
$$

depending on whether the alarm is for the system, or the cedent or the reinsurer.

In either case, we may consider extensions to 'many cedents - 1 reinsurer' model where the alarm may be designed on the basis of risk process status of the reinsurer and/or some of poor-conditioned cedents:

$$
T=\inf \left\{s>0: P\left[T^{r} \leq s+d \mid T^{r}>s, \min _{i} T^{c_{i}}<s\right] \geq 1-\alpha\right\}
$$

\section{Conclusion}

A simple model has been chosen to illustrate the notion of alarm times as well as alarm systems, and their use to alleviate the initial capital, adding a complementary capital whenever an alarm would ring. To validate such a strategy with alarm systems, comparisons have been made between a model with alarms and one without, with equivalent total capital, numerically as well as analytically, providing bounds for the difference of ruin probabilities of the two models.

Our approach has the advantage of being simple and based only on the knowledge on ruin times distributions. Hence it is adaptable to more general models using various Lévy Processes. This would include cases when the claims are dependent and/or possibly changing distribution, or when the inter-claim time spans follow more complex pattern. However, the specific performance of such an alarm system needs to be closely examined.

Through these adaptations, the proposed alarm system may also be useful for reinsurance companies. Additional considerations and variations in the alarm time formulation for the reinsurance context have been sketched. Other approaches to define alarm times and alarm systems have also been tackled, in particular when considering an EVT approach. 
As is well known, (re-) insurance institutions are mandated to periodically monitor and adjust capital capital, according to Solvency guidelines. Such reality may be easily integrated into our proposed alarm system by considering a piecewise liner accumulation function, as opposed to a linear one. The exact performance of the alarm system for such an adaptation might be worthwhile to consider.

Another direction where the current work will be expanded is in terms of moving from known deterministic models to a framework where data, as periodically received by the company, would be continually updated and fed into the current framework. This would result in formalizing a more realistic and adaptive alarm system which takes into account all data available up to current time. In principle, such an adaptation from fixed distribution for the severity structure may come via any density estimation procedure, either completely empirical based on available data or an estimate in a Bayesian paradigm. The performance of our alarm system for such an adaptation is obviously of great interest and is in the scope of our future work.

Acknowledgement. Bulk of this work was carried out during the first author's stay at ESSEC Business School and the second author's stay at the Indian Institute of Management Bangalore; the authors would like to thank both the institutions for hospitality and facilitating the research undertaken.

\section{References}

[1] Albrecher, H. and Teugels, J.L. (2007). On excess of loss Reinsurance. Eurandom technical report 17.

[2] Asmussen, S. (2000). Ruin probabilities. World Scientific.

[3] Besson, J-L, Dacorogna, M., de Martin, P., Kastenholz, M. and Moller, M. (2009). How much capital does a reinsurance need? The Geneva Papers 34 159-174.

[4] Borchani, A. (2008). Statistiques des valeurs extrêmes dans le cas de lois discrètes. ESSEC technical report.

[5] Dickson, D.C. (2005). Insurance Risk and Ruin. Cambridge.

[6] Embrechts, P., Klüppelberg, C. and Mikosch, T. (2001). Modelling extremal events for Insurance and Finance. Springer.

[7] Guillou, A., Kratz, M. and Le Strat, Y. (2010). An Extreme Value Theory approach for the early detection of time clusters with application to the surveillance of Salmonella. Preprint ArXiv 1003.4466

[8] Guillou, A., Naveau, P., Diebolt J. and Ribereau, P. (2009). Return level bounds for small and moderate sample sizes for discrete and continuous random variables Test 18, 584-604.

[9] Ignatov, Z.G and Kaishev, V.K. (2004). A finite-time ruin probability formula for continuous claim severities. J. Appl. Probab. 41 570-578.

[10] Ignatov, Z.G and Kaishev, V.K. (2006). An explicit formula for the infinite horizon probability of (non-)ruin for integer valued claims. J. Appl. Probab. 43 535-551.

[11] Ignatov, Z.G, Kaishev, V.K. and Krachunov, R.S. (2001). An improved finite-time ruin probability formula and its Mathematica implementation. Insurance: Mathematics and Enconomics $39376-389$. 
[12] Kaishev, V.K. and Dimitrova, S.D. (2006). Excess of loss reinsurance under joint survival optimality. Insurance: Mathematics and Economics 39 (3) 376-389.

[13] Kaishev, V.K., Dimitrova, S.D. and Ignatov, Z.G. (2007). Operational Risk and Insurance: a ruin-probabilistic reserving approach. Astin 2007.

[14] Lindgren, G. (1980). Model processes in non-linear prediction, with application to detection and alarm. Ann. Probab. 8 775-792.

[15] McNeil, A.J., Frey R. and Embrechts, P. (2005). Quantitative Risk Management. Princeton.

[16] Mikosch, T. (2004). Non-life Insurance Mathematics. Springer.

[17] Monteiro, M., Pereira, I. and Scotto, M. (2008). Optimal alarm systems for count processes. Communications in Statistics - Theory and Methods 37 (19) 3054-3076.

[18] Schmidli, H. (2000) Characteristic of ruin probabilities in classical risk models with and without investment, Cox risk models and perturbed risk models, Memoirs No 15, Dept. Theor. Statist., Aarhus University.

\section{A Alarm Times in Examples 1,2 and 3: Variability with Alarm Parameters $\alpha, \beta$ and $d$ :}

Table A.1.1: Alarm times with $\beta=0.025$, for different $d$ and $\alpha$ Example 1: Exponential Claim Severity

\begin{tabular}{|c|ccccccccc|}
\hline $\mathrm{d}$ & & & & & $\alpha$ & & & & \\
& $\mathbf{0 . 3}$ & $\mathbf{0 . 3 2 5}$ & $\mathbf{0 . 3 5}$ & $\mathbf{0 . 3 7 5}$ & $\mathbf{0 . 4}$ & $\mathbf{0 . 4 2 5}$ & $\mathbf{0 . 4 5}$ & $\mathbf{0 . 4 7 5}$ & $\mathbf{0 . 5}$ \\
0.75 & $\mathrm{NA}$ & NA & NA & NA & NA & NA & NA & NA & 0.19 \\
0.8 & NA & NA & NA & NA & NA & NA & NA & 0.18 & 0.12 \\
0.85 & NA & NA & NA & NA & NA & NA & 0.18 & 0.11 & 0.06 \\
0.9 & NA & NA & NA & NA & NA & 0.18 & 0.11 & 0.05 & 0 \\
0.95 & NA & NA & NA & NA & 0.18 & 0.1 & 0.05 & 0 & 0 \\
1 & NA & NA & NA & 0.18 & 0.11 & 0.05 & 0 & 0 & 0 \\
1.05 & NA & NA & 0.2 & 0.11 & 0.05 & 0 & 0 & 0 & 0 \\
1.1 & NA & NA & 0.12 & 0.06 & 0 & 0 & 0 & 0 & 0 \\
1.15 & NA & 0.14 & 0.06 & 0 & 0 & 0 & 0 & 0 & 0 \\
\hline
\end{tabular}

Table A.1.2: Alarm times with $\beta=0.075$, for different $d$ and $\alpha$ Example 1: Exponential Claim Severity

\begin{tabular}{|c|ccccccccc|}
\hline $\mathrm{d}$ & & & & & $\alpha$ & & & & \\
& $\mathbf{0 . 3}$ & $\mathbf{0 . 3 2 5}$ & $\mathbf{0 . 3 5}$ & $\mathbf{0 . 3 7 5}$ & $\mathbf{0 . 4}$ & $\mathbf{0 . 4 2 5}$ & $\mathbf{0 . 4 5}$ & $\mathbf{0 . 4 7 5}$ & $\mathbf{0 . 5}$ \\
0.7 & NA & NA & NA & NA & NA & NA & NA & NA & NA \\
0.75 & NA & NA & NA & NA & NA & NA & NA & NA & 0.19 \\
0.8 & NA & NA & NA & NA & NA & NA & NA & 0.18 & 0.12 \\
0.85 & NA & NA & NA & NA & NA & NA & 0.18 & 0.11 & 0.06 \\
0.9 & NA & NA & NA & NA & NA & 0.18 & 0.11 & 0.05 & 0 \\
0.95 & NA & NA & NA & NA & 0.18 & 0.1 & 0.05 & 0 & 0 \\
1 & NA & NA & NA & 0.18 & 0.11 & 0.05 & 0 & 0 & 0 \\
1.05 & NA & NA & 0.2 & 0.11 & 0.05 & 0 & 0 & 0 & 0 \\
1.1 & NA & 0.22 & 0.12 & 0.06 & 0 & 0 & 0 & 0 & 0 \\
1.15 & NA & 0.14 & 0.06 & 0 & 0 & 0 & 0 & 0 & 0 \\
\hline
\end{tabular}


Table A.1.3: Alarm times with $\beta=0.1$, for different $d$ and $\alpha$ Example 1: Exponential Claim Severity

\begin{tabular}{|c|c|c|c|c|c|c|c|c|c|c|}
\hline \multirow[t]{2}{*}{ d } & \multicolumn{10}{|c|}{$\alpha$} \\
\hline & 0.275 & 0.3 & 0.325 & 0.35 & 0.375 & 0.4 & 0.425 & 0.45 & 0.475 & 0.5 \\
\hline 0.7 & NA & NA & NA & NA & NA & NA & NA & NA & NA & 0.29 \\
\hline 0.75 & NA & NA & NA & NA & NA & NA & NA & NA & 0.27 & 0.19 \\
\hline 0.8 & NA & NA & NA & NA & NA & NA & NA & 0.27 & 0.18 & 0.12 \\
\hline 0.85 & NA & NA & NA & NA & NA & NA & 0.27 & 0.18 & 0.11 & 0.06 \\
\hline 0.9 & NA & NA & NA & NA & NA & 0.27 & 0.18 & 0.11 & 0.05 & 0 \\
\hline 0.95 & NA & NA & NA & NA & 0.27 & 0.18 & 0.1 & 0.05 & 0 & 0 \\
\hline 1 & NA & NA & NA & 0.29 & 0.18 & 0.11 & 0.05 & 0 & 0 & 0 \\
\hline 1.05 & NA & NA & NA & 0.2 & 0.11 & 0.05 & 0 & 0 & 0 & 0 \\
\hline 1.1 & NA & NA & 0.22 & 0.12 & 0.06 & 0 & 0 & 0 & 0 & 0 \\
\hline 1.15 & NA & 0.26 & 0.14 & 0.06 & 0 & 0 & 0 & 0 & 0 & 0 \\
\hline
\end{tabular}

Table A.1.4: Alarm times with $\beta=0.125$, for different $d$ and $\alpha$ Example 1: Exponential Claim Severity

\begin{tabular}{|c|c|c|c|c|c|c|c|c|c|c|}
\hline \multirow[t]{2}{*}{$\mathrm{d}$} & \multicolumn{10}{|c|}{$\alpha$} \\
\hline & 0.275 & 0.3 & 0.325 & 0.35 & 0.375 & 0.4 & 0.425 & 0.45 & 0.475 & 0.5 \\
\hline 0.7 & NA & NA & NA & NA & NA & NA & NA & NA & NA & 0.29 \\
\hline 0.75 & NA & NA & NA & NA & NA & NA & NA & NA & 0.27 & 0.19 \\
\hline 0.8 & NA & NA & $\mathrm{NA}$ & NA & NA & NA & NA & 0.27 & 0.18 & 0.12 \\
\hline 0.85 & NA & NA & NA & NA & NA & NA & 0.27 & 0.18 & 0.11 & 0.06 \\
\hline 0.9 & NA & NA & NA & NA & NA & 0.27 & 0.18 & 0.11 & 0.05 & 0 \\
\hline 0.95 & NA & NA & NA & NA & 0.27 & 0.18 & 0.1 & 0.05 & 0 & 0 \\
\hline 1 & NA & NA & NA & 0.29 & 0.18 & 0.11 & 0.05 & 0 & 0 & 0 \\
\hline 1.05 & NA & NA & 0.33 & 0.2 & 0.11 & 0.05 & 0 & 0 & 0 & 0 \\
\hline 1.1 & NA & NA & 0.22 & 0.12 & 0.06 & 0 & 0 & 0 & 0 & 0 \\
\hline 1.15 & NA & 0.26 & 0.14 & 0.06 & 0 & 0 & 0 & 0 & 0 & 0 \\
\hline
\end{tabular}

Table A.1.5: Alarm times with $\beta=0.15$, for different $d$ and $\alpha$ Example 1: Exponential Claim Severity

\begin{tabular}{|c|c|c|c|c|c|c|c|c|c|c|}
\hline \multirow[t]{2}{*}{$\mathrm{d}$} & \multicolumn{10}{|c|}{$\alpha$} \\
\hline & 0.275 & 0.3 & 0.325 & 0.35 & 0.375 & 0.4 & 0.425 & 0.45 & 0.475 & 0.5 \\
\hline 0.7 & NA & NA & NA & NA & NA & NA & NA & NA & NA & 0.29 \\
\hline 0.75 & NA & NA & NA & NA & NA & NA & NA & NA & 0.27 & 0.19 \\
\hline 0.8 & NA & NA & NA & NA & NA & NA & NA & 0.27 & 0.18 & 0.12 \\
\hline 0.85 & NA & NA & NA & NA & NA & NA & 0.27 & 0.18 & 0.11 & 0.06 \\
\hline 0.9 & NA & NA & NA & NA & NA & 0.27 & 0.18 & 0.11 & 0.05 & 0 \\
\hline 0.95 & NA & NA & NA & NA & 0.27 & 0.18 & 0.1 & 0.05 & 0 & 0 \\
\hline 1 & NA & NA & NA & 0.29 & 0.18 & 0.11 & 0.05 & 0 & 0 & 0 \\
\hline 1.05 & NA & NA & 0.33 & 0.2 & 0.11 & 0.05 & 0 & 0 & 0 & 0 \\
\hline 1.1 & NA & 0.37 & 0.22 & 0.12 & 0.06 & 0 & 0 & 0 & 0 & 0 \\
\hline 1.15 & NA & 0.26 & 0.14 & 0.06 & 0 & 0 & 0 & 0 & 0 & 0 \\
\hline
\end{tabular}

Table A.1.6: Alarm times with $\beta=0.175$, for different $d$ and $\alpha$ Example 1: Exponential Claim Severity

\begin{tabular}{|c|c|c|c|c|c|c|c|c|c|c|}
\hline \multirow[t]{2}{*}{$\mathrm{d}$} & \multicolumn{10}{|c|}{$\alpha$} \\
\hline & 0.275 & 0.3 & 0.325 & 0.35 & 0.375 & 0.4 & 0.425 & 0.45 & 0.475 & 0.5 \\
\hline 0.7 & NA & NA & NA & NA & NA & NA & NA & NA & NA & 0.29 \\
\hline 0.75 & NA & NA & NA & NA & NA & NA & NA & 0.4 & 0.27 & 0.19 \\
\hline 0.8 & NA & NA & NA & NA & NA & NA & 0.39 & 0.27 & 0.18 & 0.12 \\
\hline 0.85 & NA & NA & NA & NA & NA & 0.39 & 0.27 & 0.18 & 0.11 & 0.06 \\
\hline 0.9 & NA & NA & NA & NA & NA & 0.27 & 0.18 & 0.11 & 0.05 & 0 \\
\hline 0.95 & NA & NA & NA & NA & 0.27 & 0.18 & 0.1 & 0.05 & 0 & 0 \\
\hline 1 & NA & NA & NA & 0.29 & 0.18 & 0.11 & 0.05 & 0 & 0 & 0 \\
\hline 1.05 & NA & NA & 0.33 & 0.2 & 0.11 & 0.05 & 0 & 0 & 0 & 0 \\
\hline 1.1 & NA & 0.37 & 0.22 & 0.12 & 0.06 & 0 & 0 & 0 & 0 & 0 \\
\hline 1.15 & NA & 0.26 & 0.14 & 0.06 & 0 & 0 & 0 & 0 & 0 & 0 \\
\hline
\end{tabular}


Table A.1.7: Alarm times with $\beta=0.2$, for different $d$ and $\alpha$ Example 1: Exponential Claim Severity

\begin{tabular}{|c|c|c|c|c|c|c|c|c|c|c|}
\hline \multirow[t]{2}{*}{ d } & \multicolumn{10}{|c|}{$\alpha$} \\
\hline & 0.275 & 0.3 & 0.325 & 0.35 & 0.375 & 0.4 & 0.425 & 0.45 & 0.475 & 0.5 \\
\hline 0.65 & NA & NA & NA & NA & NA & NA & NA & NA & NA & 0.44 \\
\hline 0.7 & NA & NA & NA & NA & NA & NA & NA & NA & 0.42 & 0.29 \\
\hline 0.75 & NA & NA & NA & NA & NA & NA & NA & 0.4 & 0.27 & 0.19 \\
\hline 0.8 & NA & NA & NA & NA & NA & NA & 0.39 & 0.27 & 0.18 & 0.12 \\
\hline 0.85 & NA & NA & NA & NA & NA & 0.39 & 0.27 & 0.18 & 0.11 & 0.06 \\
\hline 0.9 & NA & NA & NA & NA & 0.42 & 0.27 & 0.18 & 0.11 & 0.05 & 0 \\
\hline 0.95 & NA & NA & NA & NA & 0.27 & 0.18 & 0.1 & 0.05 & 0 & 0 \\
\hline 1 & NA & NA & NA & 0.29 & 0.18 & 0.11 & 0.05 & 0 & 0 & 0 \\
\hline 1.05 & NA & NA & 0.33 & 0.2 & 0.11 & 0.05 & 0 & 0 & 0 & 0 \\
\hline 1.1 & NA & 0.37 & 0.22 & 0.12 & 0.06 & 0 & 0 & 0 & 0 & 0 \\
\hline 1.15 & NA & 0.26 & 0.14 & 0.06 & 0 & 0 & 0 & 0 & 0 & 0 \\
\hline
\end{tabular}

Table A.1.8: Alarm times with $\beta=0.225$, for different $d$ and $\alpha$ Example 1: Exponential Claim Severity

\begin{tabular}{|c|c|c|c|c|c|c|c|c|c|c|}
\hline \multirow[t]{2}{*}{$\mathrm{d}$} & \multicolumn{10}{|c|}{$\alpha$} \\
\hline & 0.275 & 0.3 & 0.325 & 0.35 & 0.375 & 0.4 & 0.425 & 0.45 & 0.475 & 0.5 \\
\hline 0.65 & NA & NA & NA & NA & NA & NA & NA & NA & NA & 0.44 \\
\hline 0.7 & NA & NA & NA & NA & NA & NA & NA & NA & 0.42 & 0.29 \\
\hline 0.75 & NA & NA & NA & NA & NA & NA & NA & 0.4 & 0.27 & 0.19 \\
\hline 0.8 & NA & NA & NA & NA & NA & NA & 0.39 & 0.27 & 0.18 & 0.12 \\
\hline 0.85 & NA & NA & NA & NA & NA & 0.39 & 0.27 & 0.18 & 0.11 & 0.06 \\
\hline 0.9 & NA & NA & NA & NA & 0.42 & 0.27 & 0.18 & 0.11 & 0.05 & 0 \\
\hline 0.95 & NA & NA & NA & 0.45 & 0.27 & 0.18 & 0.1 & 0.05 & 0 & 0 \\
\hline 1 & NA & NA & NA & 0.29 & 0.18 & 0.11 & 0.05 & 0 & 0 & 0 \\
\hline 1.05 & NA & NA & 0.33 & 0.2 & 0.11 & 0.05 & 0 & 0 & 0 & 0 \\
\hline 1.1 & NA & 0.37 & 0.22 & 0.12 & 0.06 & 0 & 0 & 0 & 0 & 0 \\
\hline 1.15 & 0.46 & 0.26 & 0.14 & 0.06 & 0 & 0 & 0 & 0 & 0 & 0 \\
\hline
\end{tabular}

Table A.2.1: Alarm times with $\beta=0.075$ and 0.1, for different $d$ and $\alpha$ Example 2: Pareto Claim Severity

\begin{tabular}{|c|c|c|c|c|c|c|c|c|c|c|}
\hline & \multicolumn{4}{|c|}{$\beta=0.075$} & & \multicolumn{5}{|c|}{$\beta=0.1$} \\
\hline $\mathrm{d}$ & & & & & & & & $\alpha$ & & \\
\hline & 0.4 & 0.425 & 0.45 & 0.475 & d & 0.375 & 0.4 & 0.425 & 0.45 & 0.475 \\
\hline 0.95 & NA & NA & NA & NA & 0.95 & NA & NA & NA & NA & NA \\
\hline 1 & NA & NA & NA & 0.07 & 1 & NA & NA & NA & NA & 0.07 \\
\hline 1.05 & NA & NA & 0.09 & 0 & 1.05 & NA & NA & NA & 0.09 & 0 \\
\hline 1.1 & NA & 0.13 & 0 & 0 & 1.1 & NA & NA & 0.13 & 0 & 0 \\
\hline 1.15 & NA & 0.01 & 0 & 0 & 1.15 & NA & 0.18 & 0.01 & 0 & 0 \\
\hline
\end{tabular}

Table A.2.2: Alarm times with $\beta=0.125$ and 0.15 , for different $d$ and $\alpha$ Example 2: Pareto Claim Severity

\begin{tabular}{|c|c|c|c|c|c|c|c|c|c|c|c|}
\hline & \multicolumn{5}{|c|}{$\beta=0.125$} & & \multicolumn{5}{|c|}{$\beta=0.15$} \\
\hline \multirow[t]{2}{*}{$\mathrm{d}$} & \multicolumn{5}{|c|}{$\alpha$} & & & & $\alpha$ & & \\
\hline & 0.375 & 0.4 & 0.425 & 0.45 & 0.475 & $\mathrm{~d}$ & 0.375 & 0.4 & 0.425 & 0.45 & 0.475 \\
\hline 0.9 & NA & NA & NA & NA & NA & 0.9 & NA & NA & NA & NA & NA \\
\hline 0.95 & NA & NA & NA & NA & 0.21 & 0.95 & NA & NA & NA & NA & 0.21 \\
\hline 1 & NA & NA & NA & NA & 0.07 & 1 & NA & NA & NA & 0.24 & 0.07 \\
\hline 1.05 & NA & NA & NA & 0.09 & 0 & 1.05 & NA & NA & 0.28 & 0.09 & 0 \\
\hline 1.1 & NA & NA & 0.13 & 0 & 0 & 1.1 & NA & NA & 0.13 & 0 & 0 \\
\hline 1.15 & NA & 0.18 & 0.01 & 0 & 0 & 1.15 & NA & 0.18 & 0.01 & 0 & 0 \\
\hline
\end{tabular}


Table A.2.3: Alarm times with $\beta=0.175$ and 0.2, for different $d$ and $\alpha$ Example 2: Pareto Claim Severity

\begin{tabular}{|c|c|c|c|c|c|c|c|c|c|c|c|}
\hline & \multicolumn{5}{|c|}{$\beta=0.175$} & & \multicolumn{5}{|c|}{$\beta=0.2$} \\
\hline \multirow[t]{2}{*}{$\mathrm{d}$} & \multicolumn{5}{|c|}{$\alpha$} & \multirow[b]{2}{*}{$\mathrm{d}$} & \multicolumn{5}{|c|}{$\alpha$} \\
\hline & 0.375 & 0.4 & 0.425 & 0.45 & 0.475 & & 0.375 & 0.4 & 0.425 & 0.45 & 0.475 \\
\hline 0.9 & NA & NA & NA & NA & NA & 0.9 & NA & NA & NA & NA & NA \\
\hline 0.95 & NA & NA & NA & NA & 0.21 & 0.95 & NA & NA & NA & NA & 0.21 \\
\hline 1 & NA & NA & NA & 0.24 & 0.07 & 1 & NA & NA & NA & 0.24 & 0.07 \\
\hline 1.05 & NA & NA & 0.28 & 0.09 & 0 & 1.05 & NA & NA & 0.28 & 0.09 & 0 \\
\hline 1.1 & NA & NA & 0.13 & 0 & 0 & 1.1 & NA & 0.35 & 0.13 & 0 & 0 \\
\hline 1.15 & NA & 0.18 & 0.01 & 0 & 0 & 1.15 & NA & 0.18 & 0.01 & 0 & 0 \\
\hline
\end{tabular}

Table A.3.1: Alarm times with $\beta=0.05$, for different $d$ and $\alpha$ Example 3: Discrete Logarithm Claim Severity

\begin{tabular}{|c|c|c|c|c|c|c|c|c|c|c|}
\hline \multirow[t]{2}{*}{$\mathrm{d}$} & \multicolumn{10}{|c|}{$\alpha$} \\
\hline & 0.25 & 0.275 & 0.3 & 0.325 & 0.35 & 0.375 & 0.4 & 0.425 & 0.45 & 0.475 \\
\hline 0.25 & NA & NA & NA & NA & NA & NA & NA & NA & NA & NA \\
\hline 0.27 & NA & NA & NA & NA & NA & NA & NA & NA & NA & NA \\
\hline 0.29 & NA & NA & NA & NA & NA & NA & NA & NA & NA & 0.07 \\
\hline 0.31 & NA & NA & NA & NA & NA & NA & NA & 0.08 & 0.06 & 0.05 \\
\hline 0.33 & NA & NA & NA & NA & NA & NA & 0.07 & 0.06 & 0.04 & 0.03 \\
\hline 0.35 & NA & NA & NA & NA & 0.08 & 0.07 & 0.05 & 0.03 & 0.02 & 0 \\
\hline 0.37 & NA & NA & NA & 0.08 & 0.06 & 0.04 & 0.03 & 0.01 & 0 & 0 \\
\hline 0.39 & NA & NA & 0.07 & 0.05 & 0.04 & 0.02 & 0.01 & 0 & 0 & 0 \\
\hline 0.41 & NA & 0.07 & 0.05 & 0.03 & 0.02 & 0 & 0 & 0 & 0 & 0 \\
\hline 0.43 & 0.07 & 0.05 & 0.03 & 0.01 & 0 & 0 & 0 & 0 & 0 & 0 \\
\hline 0.45 & 0.04 & 0.02 & 0.01 & 0 & 0 & 0 & 0 & 0 & 0 & 0 \\
\hline 0.47 & 0.03 & 0.01 & 0 & 0 & 0 & 0 & 0 & 0 & 0 & 0 \\
\hline 0.49 & 0 & 0 & 0 & 0 & 0 & 0 & 0 & 0 & 0 & 0 \\
\hline
\end{tabular}

Table A.3.2: Alarm times with $\beta=0.075$, for different $d$ and $\alpha$ Example 3: Discrete Logarithm Claim Severity

\begin{tabular}{|c|c|c|c|c|c|c|c|c|c|c|}
\hline \multirow[t]{2}{*}{$\mathrm{d}$} & \multicolumn{10}{|c|}{$\alpha$} \\
\hline & 0.25 & 0.275 & 0.3 & 0.325 & 0.35 & 0.375 & 0.4 & 0.425 & 0.45 & 0.475 \\
\hline 0.25 & NA & NA & NA & NA & NA & NA & NA & NA & NA & NA \\
\hline 0.27 & NA & NA & NA & NA & NA & NA & NA & NA & NA & 0.1 \\
\hline 0.29 & NA & NA & NA & NA & NA & NA & NA & NA & 0.09 & 0.07 \\
\hline 0.31 & NA & $\mathrm{NA}$ & NA & NA & NA & $\mathrm{NA}$ & 0.1 & 0.08 & 0.06 & 0.05 \\
\hline 0.33 & NA & NA & NA & NA & NA & 0.09 & 0.07 & 0.06 & 0.04 & 0.03 \\
\hline 0.35 & NA & NA & NA & 0.1 & 0.08 & 0.07 & 0.05 & 0.03 & 0.02 & 0 \\
\hline 0.37 & NA & NA & 0.1 & 0.08 & 0.06 & 0.04 & 0.03 & 0.01 & 0 & 0 \\
\hline 0.39 & NA & 0.09 & 0.07 & 0.05 & 0.04 & 0.02 & 0.01 & 0 & 0 & 0 \\
\hline 0.41 & 0.09 & 0.07 & 0.05 & 0.03 & 0.02 & 0 & 0 & 0 & 0 & 0 \\
\hline 0.43 & 0.07 & 0.05 & 0.03 & 0.01 & 0 & 0 & 0 & 0 & 0 & 0 \\
\hline 0.45 & 0.04 & 0.02 & 0.01 & 0 & 0 & 0 & 0 & 0 & 0 & 0 \\
\hline 0.47 & 0.03 & 0.01 & 0 & 0 & 0 & 0 & 0 & 0 & 0 & 0 \\
\hline 0.49 & 0 & 0 & 0 & 0 & 0 & 0 & 0 & 0 & 0 & 0 \\
\hline
\end{tabular}


Table A.3.3: Alarm times with $\beta=0.1$, for different $d$ and $\alpha$ Example 3: Discrete Logarithm Claim Severity

\begin{tabular}{|c|c|c|c|c|c|c|c|c|c|c|}
\hline \multirow[t]{2}{*}{ d } & \multicolumn{10}{|c|}{$\alpha$} \\
\hline & 0.25 & 0.275 & 0.3 & 0.325 & 0.35 & 0.375 & 0.4 & 0.425 & 0.45 & 0.475 \\
\hline 0.25 & NA & NA & NA & NA & NA & NA & NA & NA & NA & NA \\
\hline 0.27 & NA & NA & NA & NA & NA & NA & NA & NA & 0.12 & 0.1 \\
\hline 0.29 & NA & NA & NA & NA & NA & NA & NA & 0.11 & 0.09 & 0.07 \\
\hline 0.31 & NA & NA & NA & NA & NA & 0.12 & 0.1 & 0.08 & 0.06 & 0.05 \\
\hline 0.33 & NA & NA & NA & NA & 0.11 & 0.09 & 0.07 & 0.06 & 0.04 & 0.03 \\
\hline 0.35 & NA & NA & NA & 0.1 & 0.08 & 0.07 & 0.05 & 0.03 & 0.02 & 0 \\
\hline 0.37 & NA & 0.12 & 0.1 & 0.08 & 0.06 & 0.04 & 0.03 & 0.01 & 0 & 0 \\
\hline 0.39 & 0.12 & 0.09 & 0.07 & 0.05 & 0.04 & 0.02 & 0.01 & 0 & 0 & 0 \\
\hline 0.41 & 0.09 & 0.07 & 0.05 & 0.03 & 0.02 & 0 & 0 & 0 & 0 & 0 \\
\hline 0.43 & 0.07 & 0.05 & 0.03 & 0.01 & 0 & 0 & 0 & 0 & 0 & 0 \\
\hline 0.45 & 0.04 & 0.02 & 0.01 & 0 & 0 & 0 & 0 & 0 & 0 & 0 \\
\hline 0.47 & 0.03 & 0.01 & 0 & 0 & 0 & 0 & 0 & 0 & 0 & 0 \\
\hline 0.49 & 0 & 0 & 0 & 0 & 0 & 0 & 0 & 0 & 0 & 0 \\
\hline
\end{tabular}

Table A.3.4: Alarm times with $\beta=0.125$, for different $d$ and $\alpha$ Example 3: Discrete Logarithm Claim Severity

\begin{tabular}{|c|c|c|c|c|c|c|c|c|c|c|}
\hline \multirow[t]{2}{*}{$\mathrm{d}$} & \multicolumn{10}{|c|}{$\alpha$} \\
\hline & 0.25 & 0.275 & 0.3 & 0.325 & 0.35 & 0.375 & 0.4 & 0.425 & 0.45 & 0.475 \\
\hline 0.25 & NA & NA & NA & NA & NA & NA & NA & NA & NA & 0.13 \\
\hline 0.27 & NA & NA & NA & NA & NA & NA & NA & 0.14 & 0.12 & 0.1 \\
\hline 0.29 & NA & NA & NA & NA & NA & NA & 0.13 & 0.11 & 0.09 & 0.07 \\
\hline 0.31 & NA & NA & NA & NA & 0.14 & 0.12 & 0.1 & 0.08 & 0.06 & 0.05 \\
\hline 0.33 & NA & NA & NA & 0.13 & 0.11 & 0.09 & 0.07 & 0.06 & 0.04 & 0.03 \\
\hline 0.35 & NA & NA & 0.13 & 0.1 & 0.08 & 0.07 & 0.05 & 0.03 & 0.02 & 0 \\
\hline 0.37 & NA & 0.12 & 0.1 & 0.08 & 0.06 & 0.04 & 0.03 & 0.01 & 0 & 0 \\
\hline 0.39 & 0.12 & 0.09 & 0.07 & 0.05 & 0.04 & 0.02 & 0.01 & 0 & 0 & 0 \\
\hline 0.41 & 0.09 & 0.07 & 0.05 & 0.03 & 0.02 & 0 & 0 & 0 & 0 & 0 \\
\hline 0.43 & 0.07 & 0.05 & 0.03 & 0.01 & 0 & 0 & 0 & 0 & 0 & 0 \\
\hline 0.45 & 0.04 & 0.02 & 0.01 & 0 & 0 & 0 & 0 & 0 & 0 & 0 \\
\hline 0.47 & 0.03 & 0.01 & 0 & 0 & 0 & 0 & 0 & 0 & 0 & 0 \\
\hline 0.49 & 0 & 0 & 0 & 0 & 0 & 0 & 0 & 0 & 0 & 0 \\
\hline
\end{tabular}

Table A.3.5: Alarm times with $\beta=0.15$, for different $d$ and $\alpha$ Example 3: Discrete Logarithm Claim Severity

\begin{tabular}{|c|c|c|c|c|c|c|c|c|c|c|}
\hline \multirow[t]{2}{*}{$\mathrm{d}$} & \multicolumn{10}{|c|}{$\alpha$} \\
\hline & 0.25 & 0.275 & 0.3 & 0.325 & 0.35 & 0.375 & 0.4 & 0.425 & 0.45 & 0.475 \\
\hline 0.25 & NA & NA & NA & NA & NA & NA & NA & NA & 0.15 & 0.13 \\
\hline 0.27 & NA & NA & NA & NA & NA & NA & NA & 0.14 & 0.12 & 0.1 \\
\hline 0.29 & NA & NA & NA & NA & NA & 0.15 & 0.13 & 0.11 & 0.09 & 0.07 \\
\hline 0.31 & NA & NA & NA & NA & 0.14 & 0.12 & 0.1 & 0.08 & 0.06 & 0.05 \\
\hline 0.33 & NA & NA & 0.15 & 0.13 & 0.11 & 0.09 & 0.07 & 0.06 & 0.04 & 0.03 \\
\hline 0.35 & NA & 0.15 & 0.13 & 0.1 & 0.08 & 0.07 & 0.05 & 0.03 & 0.02 & 0 \\
\hline 0.37 & 0.15 & 0.12 & 0.1 & 0.08 & 0.06 & 0.04 & 0.03 & 0.01 & 0 & 0 \\
\hline 0.39 & 0.12 & 0.09 & 0.07 & 0.05 & 0.04 & 0.02 & 0.01 & 0 & 0 & 0 \\
\hline 0.41 & 0.09 & 0.07 & 0.05 & 0.03 & 0.02 & 0 & 0 & 0 & 0 & 0 \\
\hline 0.43 & 0.07 & 0.05 & 0.03 & 0.01 & 0 & 0 & 0 & 0 & 0 & 0 \\
\hline 0.45 & 0.04 & 0.02 & 0.01 & 0 & 0 & 0 & 0 & 0 & 0 & 0 \\
\hline 0.47 & 0.03 & 0.01 & 0 & 0 & 0 & 0 & 0 & 0 & 0 & 0 \\
\hline 0.49 & 0 & 0 & 0 & 0 & 0 & 0 & 0 & 0 & 0 & 0 \\
\hline
\end{tabular}


Table A.3.6: Alarm times with $\beta=0.175$, for different $d$ and $\alpha$ Example 3: Discrete Logarithm Claim Severity

\begin{tabular}{|c|ccccccccccc|}
\hline $\mathrm{d}$ & $\mathbf{8}$ & $\mathbf{0 . 2 5}$ & $\mathbf{0 . 2 7 5}$ & $\mathbf{0 . 3}$ & $\mathbf{0 . 3 2 5}$ & $\mathbf{0 . 3 5}$ & $\mathbf{0 . 3 7 5}$ & $\mathbf{0 . 4}$ & $\mathbf{0 . 4 2 5}$ & $\mathbf{0 . 4 5}$ & $\mathbf{0 . 4 7 5}$ \\
& $\mathbf{0 . 4}$ & NA & NA & NA & NA & NA & NA & NA & 0.17 & 0.15 & 0.13 \\
0.27 & NA & NA & NA & NA & NA & NA & 0.16 & 0.14 & 0.12 & 0.1 \\
0.29 & NA & NA & NA & NA & 0.17 & 0.15 & 0.13 & 0.11 & 0.09 & 0.07 \\
0.31 & NA & NA & NA & 0.16 & 0.14 & 0.12 & 0.1 & 0.08 & 0.06 & 0.05 \\
0.33 & NA & NA & 0.15 & 0.13 & 0.11 & 0.09 & 0.07 & 0.06 & 0.04 & 0.03 \\
0.35 & NA & 0.15 & 0.13 & 0.1 & 0.08 & 0.07 & 0.05 & 0.03 & 0.02 & 0 \\
0.37 & 0.15 & 0.12 & 0.1 & 0.08 & 0.06 & 0.04 & 0.03 & 0.01 & 0 & 0 \\
0.39 & 0.12 & 0.09 & 0.07 & 0.05 & 0.04 & 0.02 & 0.01 & 0 & 0 & 0 \\
0.41 & 0.09 & 0.07 & 0.05 & 0.03 & 0.02 & 0 & 0 & 0 & 0 & 0 \\
0.43 & 0.07 & 0.05 & 0.03 & 0.01 & 0 & 0 & 0 & 0 & 0 & 0 \\
0.45 & 0.04 & 0.02 & 0.01 & 0 & 0 & 0 & 0 & 0 & 0 & 0 \\
0.47 & 0.03 & 0.01 & 0 & 0 & 0 & 0 & 0 & 0 & 0 & 0 \\
\hline
\end{tabular}

Table A.3.7: Alarm times with $\beta=0.2$, for different $d$ and $\alpha$ Example 3: Discrete Logarithm Claim Severity

\begin{tabular}{|c|c|c|c|c|c|c|c|c|c|c|}
\hline \multirow[t]{2}{*}{ d } & \multicolumn{10}{|c|}{$\alpha$} \\
\hline & 0.25 & 0.275 & 0.3 & 0.325 & 0.35 & 0.375 & 0.4 & 0.425 & 0.45 & 0.475 \\
\hline 0.25 & NA & NA & NA & NA & NA & NA & NA & 0.17 & 0.15 & 0.13 \\
\hline 0.27 & NA & NA & NA & NA & NA & 0.18 & 0.16 & 0.14 & 0.12 & 0.1 \\
\hline 0.29 & NA & NA & NA & NA & 0.17 & 0.15 & 0.13 & 0.11 & 0.09 & 0.07 \\
\hline 0.31 & NA & NA & NA & 0.16 & 0.14 & 0.12 & 0.1 & 0.08 & 0.06 & 0.05 \\
\hline 0.33 & NA & 0.18 & 0.15 & 0.13 & 0.11 & 0.09 & 0.07 & 0.06 & 0.04 & 0.03 \\
\hline 0.35 & 0.18 & 0.15 & 0.13 & 0.1 & 0.08 & 0.07 & 0.05 & 0.03 & 0.02 & 0 \\
\hline 0.37 & 0.15 & 0.12 & 0.1 & 0.08 & 0.06 & 0.04 & 0.03 & 0.01 & 0 & 0 \\
\hline 0.39 & 0.12 & 0.09 & 0.07 & 0.05 & 0.04 & 0.02 & 0.01 & 0 & 0 & 0 \\
\hline 0.41 & 0.09 & 0.07 & 0.05 & 0.03 & 0.02 & 0 & 0 & 0 & 0 & 0 \\
\hline 0.43 & 0.07 & 0.05 & 0.03 & 0.01 & 0 & 0 & 0 & 0 & 0 & 0 \\
\hline 0.45 & 0.04 & 0.02 & 0.01 & 0 & 0 & 0 & 0 & 0 & 0 & 0 \\
\hline 0.47 & 0.03 & 0.01 & 0 & 0 & 0 & 0 & 0 & 0 & 0 & 0 \\
\hline 0.49 & 0 & 0 & 0 & 0 & 0 & 0 & 0 & 0 & 0 & 0 \\
\hline
\end{tabular}

Table A.3.8: Alarm times with $\beta=0.225$, for different $d$ and $\alpha$ Example 3: Discrete Logarithm Claim Severity

\begin{tabular}{|c|c|c|c|c|c|c|c|c|c|c|}
\hline \multirow[t]{2}{*}{$\mathrm{d}$} & \multicolumn{10}{|c|}{$\alpha$} \\
\hline & 0.25 & 0.275 & 0.3 & 0.325 & 0.35 & 0.375 & 0.4 & 0.425 & 0.45 & 0.475 \\
\hline 0.25 & NA & NA & NA & NA & NA & NA & 0.19 & 0.17 & 0.15 & 0.13 \\
\hline 0.27 & NA & NA & NA & NA & NA & 0.18 & 0.16 & 0.14 & 0.12 & 0.1 \\
\hline 0.29 & NA & NA & NA & NA & 0.17 & 0.15 & 0.13 & 0.11 & 0.09 & 0.07 \\
\hline 0.31 & NA & NA & 0.19 & 0.16 & 0.14 & 0.12 & 0.1 & 0.08 & 0.06 & 0.05 \\
\hline 0.33 & NA & 0.18 & 0.15 & 0.13 & 0.11 & 0.09 & 0.07 & 0.06 & 0.04 & 0.03 \\
\hline 0.35 & 0.18 & 0.15 & 0.13 & 0.1 & 0.08 & 0.07 & 0.05 & 0.03 & 0.02 & 0 \\
\hline 0.37 & 0.15 & 0.12 & 0.1 & 0.08 & 0.06 & 0.04 & 0.03 & 0.01 & 0 & 0 \\
\hline 0.39 & 0.12 & 0.09 & 0.07 & 0.05 & 0.04 & 0.02 & 0.01 & 0 & 0 & 0 \\
\hline 0.41 & 0.09 & 0.07 & 0.05 & 0.03 & 0.02 & 0 & 0 & 0 & 0 & 0 \\
\hline 0.43 & 0.07 & 0.05 & 0.03 & 0.01 & 0 & 0 & 0 & 0 & 0 & 0 \\
\hline 0.45 & 0.04 & 0.02 & 0.01 & 0 & 0 & 0 & 0 & 0 & 0 & 0 \\
\hline 0.47 & 0.03 & 0.01 & 0 & 0 & 0 & 0 & 0 & 0 & 0 & 0 \\
\hline 0.49 & 0 & 0 & 0 & 0 & 0 & 0 & 0 & 0 & 0 & 0 \\
\hline
\end{tabular}




\section{B Survival Probability Table in Example 2 (Proposition 4)}

Table B.1:

\begin{tabular}{rcccccccc}
\hline \multicolumn{10}{c}{$u^{\mathbf{c}}$} & -28.04 & -25 & -20 & -18.54 & -15 & -10 & -9.04 & -5 \\
\hline $\mathbf{0}$ & 1 & 1 & 1 & 1 & 1 & 1 & 1 & 1 \\
\hline $\mathbf{0 . 0 1}$ & 0.819 & 0.819 & 0.819 & 0.819 & 0.819 & 0.819 & 0.819 & 0.819 \\
\hline $\mathbf{0 . 0 2}$ & 0.670 & 0.670 & 0.670 & 0.670 & 0.670 & 0.670 & 0.670 & 0.670 \\
\hline $\mathbf{0 . 0 3}$ & 0.550 & 0.550 & 0.550 & 0.550 & 0.550 & 0.550 & 0.550 & 0.550 \\
\hline $\mathbf{0 . 0 4}$ & 0.450 & 0.450 & 0.450 & 0.450 & 0.450 & 0.450 & 0.450 & 0.450 \\
\hline $\mathbf{0 . 0 5}$ & 0.369 & 0.369 & 0.369 & 0.369 & 0.369 & 0.369 & 0.369 & 0.369 \\
\hline $\mathbf{0 . 0 6}$ & 0.303 & 0.303 & 0.303 & 0.303 & 0.303 & 0.303 & 0.303 & 0.303 \\
\hline $\mathbf{0 . 0 7}$ & 0.249 & 0.249 & 0.249 & 0.249 & 0.249 & 0.249 & 0.249 & 0.249 \\
\hline $\mathbf{0 . 0 8}$ & 0.204 & 0.204 & 0.204 & 0.204 & 0.204 & 0.204 & 0.204 & 0.204 \\
\hline $\mathbf{0 . 0 9}$ & 0.168 & 0.168 & 0.168 & 0.168 & 0.168 & 0.168 & 0.168 & 0.168 \\
\hline $\mathbf{0 . 1}$ & 0.137 & 0.137 & 0.137 & 0.137 & 0.137 & 0.137 & 0.137 & 0.137 \\
\hline $\mathbf{0 . 1 1}$ & 0.112 & 0.112 & 0.112 & 0.112 & 0.112 & 0.112 & 0.112 & 0.112 \\
\hline $\mathbf{0 . 1 7}$ & 0.034 & 0.034 & 0.034 & 0.034 & 0.034 & 0.034 & 0.034 & 0.049 \\
\hline $\mathbf{0 . 2}$ & 0.019 & 0.019 & 0.019 & 0.019 & 0.019 & 0.019 & 0.019 & 0.039 \\
\hline $\mathbf{0 . 2 9}$ & 0.003 & 0.003 & 0.003 & 0.003 & 0.003 & 0.004 & 0.006 & 0.026 \\
\hline $\mathbf{0 . 4}$ & 0.000 & 0.000 & 0.000 & 0.000 & 0.000 & 0.002 & 0.003 & 0.018 \\
\hline $\mathbf{0 . 5}$ & 0.000 & 0.000 & 0.000 & 0.000 & 0.000 & 0.002 & 0.002 & 0.013 \\
\hline $\mathbf{0 . 5 8}$ & 0.000 & 0.000 & 0.000 & 0.000 & 0.000 & 0.001 & 0.002 & 0.011 \\
\hline $\mathbf{0 . 7 5}$ & 0.000 & 0.000 & 0.000 & 0.000 & 0.000 & 0.001 & 0.001 & 0.008 \\
\hline $\mathbf{0 . 9}$ & 0.000 & 0.000 & 0.000 & 0.000 & 0.000 & 0.001 & 0.001 & 0.005 \\
\hline $\mathbf{1}$ & 0.000 & 0.000 & 0.000 & 0.000 & 0.000 & 0.001 & 0.001 & 0.005 \\
\hline $\mathbf{1 . 2 8}$ & 0.000 & 0.000 & 0.000 & 0.000 & 0.000 & 0.000 & 0.000 & 0.003 \\
\hline & & & & & & & &
\end{tabular}

Table B.2:

\begin{tabular}{|c|c|c|c|c|c|c|c|c|c|c|c|c|}
\hline & \multicolumn{12}{|c|}{$u_{0}$} \\
\hline $\mathbf{t}$ & 0 & 0.54 & 4.82 & 5 & 9.08 & 9.5 & 9.82 & 10 & 14.82 & 15 & 19.08 & 19.5 \\
\hline 0 & 1 & 1 & 1 & 1 & 1 & 1 & 1 & 1 & 1 & 1 & 1 & 1 \\
\hline 0.01 & 0.844 & 0.887 & 0.963 & 0.964 & 0.978 & 0.979 & 0.979 & 0.980 & 0.986 & 0.986 & 0.989 & 0.989 \\
\hline 0.02 & 0.742 & 0.803 & 0.928 & 0.930 & 0.956 & 0.957 & 0.958 & 0.959 & 0.971 & 0.971 & 0.977 & 0.977 \\
\hline 0.03 & 0.667 & 0.737 & 0.895 & 0.898 & 0.934 & 0.936 & 0.938 & 0.939 & 0.956 & 0.956 & 0.965 & 0.965 \\
\hline 0.04 & 0.609 & 0.682 & 0.864 & 0.867 & 0.913 & 0.916 & 0.918 & 0.919 & 0.941 & 0.942 & 0.953 & 0.954 \\
\hline 0.05 & 0.562 & 0.637 & 0.835 & 0.838 & 0.892 & 0.895 & 0.898 & 0.899 & 0.927 & 0.927 & 0.941 & 0.942 \\
\hline 0.06 & 0.523 & 0.597 & 0.806 & 0.810 & 0.871 & 0.875 & 0.878 & 0.880 & 0.912 & 0.913 & 0.929 & 0.930 \\
\hline 0.07 & 0.490 & 0.562 & 0.780 & 0.784 & 0.851 & 0.856 & 0.860 & 0.861 & 0.897 & 0.898 & 0.917 & 0.918 \\
\hline 0.08 & 0.461 & 0.532 & 0.754 & 0.759 & 0.832 & 0.837 & 0.841 & 0.843 & 0.883 & 0.884 & 0.905 & 0.907 \\
\hline 0.09 & 0.435 & 0.505 & 0.731 & 0.735 & 0.813 & 0.818 & 0.823 & 0.825 & 0.869 & 0.870 & 0.893 & 0.895 \\
\hline 0.1 & 0.412 & 0.480 & 0.708 & 0.713 & 0.795 & 0.801 & 0.805 & 0.808 & 0.855 & 0.856 & 0.881 & 0.884 \\
\hline 0.11 & 0.392 & 0.458 & 0.686 & 0.692 & 0.777 & 0.783 & 0.788 & 0.791 & 0.841 & 0.843 & 0.870 & 0.872 \\
\hline 0.17 & 0.301 & 0.356 & 0.575 & 0.580 & 0.680 & 0.688 & 0.693 & 0.696 & 0.762 & 0.764 & 0.801 & 0.805 \\
\hline 0.2 & 0.268 & 0.319 & 0.529 & 0.535 & 0.637 & 0.645 & 0.651 & 0.655 & 0.726 & 0.728 & 0.769 & 0.773 \\
\hline 0.29 & 0.200 & 0.240 & 0.421 & 0.427 & 0.530 & 0.538 & 0.544 & 0.548 & 0.626 & 0.628 & 0.678 & 0.682 \\
\hline 0.4 & 0.149 & 0.179 & 0.328 & 0.333 & 0.427 & 0.436 & 0.442 & 0.445 & 0.525 & 0.527 & 0.581 & 0.586 \\
\hline 0.5 & 0.117 & 0.141 & 0.266 & 0.270 & 0.355 & 0.363 & 0.369 & 0.372 & 0.448 & 0.451 & 0.505 & 0.510 \\
\hline 0.58 & 0.098 & 0.118 & 0.227 & 0.231 & 0.308 & 0.315 & 0.320 & 0.323 & 0.396 & 0.399 & 0.452 & 0.457 \\
\hline 0.75 & 0.069 & 0.084 & 0.165 & 0.168 & 0.231 & 0.236 & 0.241 & 0.243 & 0.306 & 0.308 & 0.357 & 0.362 \\
\hline 0.9 & 0.052 & 0.063 & 0.127 & 0.129 & 0.181 & 0.186 & 0.189 & 0.192 & 0.246 & 0.248 & 0.291 & 0.296 \\
\hline 1 & 0.044 & 0.054 & 0.108 & 0.110 & 0.155 & 0.159 & 0.162 & 0.164 & 0.213 & 0.215 & 0.255 & 0.259 \\
\hline 1.28 & 0.028 & 0.034 & 0.070 & 0.071 & 0.102 & 0.106 & 0.108 & 0.110 & 0.145 & 0.147 & 0.177 & 0.180 \\
\hline 1.4 & 0.023 & 0.028 & 0.058 & 0.059 & 0.086 & 0.089 & 0.091 & 0.092 & 0.123 & 0.125 & 0.152 & 0.154 \\
\hline 1.49 & 0.020 & 0.025 & 0.051 & 0.052 & 0.076 & 0.079 & 0.081 & 0.082 & 0.110 & 0.111 & 0.135 & 0.138 \\
\hline
\end{tabular}


Table B.3:

\begin{tabular}{|c|c|c|c|c|c|c|c|c|c|c|c|c|}
\hline & \multicolumn{12}{|c|}{$u_{0}$} \\
\hline $\mathrm{t}$ & 19.82 & 20 & 24.82 & 25 & 29.08 & 29.5 & 29.82 & 30 & 34.82 & 35 & 39.08 & 39.5 \\
\hline 0 & 1 & 1 & 1 & 1 & 1 & 1 & 1 & 1 & 1 & 1 & 1 & 1 \\
\hline 0.01 & 0.989 & 0.989 & 0.991 & 0.991 & 0.992 & 0.992 & 0.992 & 0.992 & 0.993 & 0.993 & 0.994 & 0.994 \\
\hline 0.02 & 0.977 & 0.977 & 0.981 & 0.982 & 0.984 & 0.984 & 0.984 & 0.984 & 0.986 & 0.986 & 0.988 & 0.988 \\
\hline 0.03 & 0.966 & 0.966 & 0.972 & 0.972 & 0.976 & 0.976 & 0.976 & 0.977 & 0.979 & 0.980 & 0.982 & 0.982 \\
\hline 0.04 & 0.954 & 0.955 & 0.963 & 0.963 & 0.968 & 0.968 & 0.968 & 0.969 & 0.973 & 0.973 & 0.975 & 0.976 \\
\hline 0.05 & 0.943 & 0.943 & 0.953 & 0.954 & 0.960 & 0.960 & 0.960 & 0.961 & 0.966 & 0.966 & 0.969 & 0.970 \\
\hline 0.06 & 0.931 & 0.932 & 0.943 & 0.944 & 0.951 & 0.952 & 0.952 & 0.952 & 0.959 & 0.959 & 0.963 & 0.963 \\
\hline 0.07 & 0.919 & 0.920 & 0.934 & 0.934 & 0.942 & 0.943 & 0.944 & 0.944 & 0.951 & 0.952 & 0.956 & 0.957 \\
\hline 0.08 & 0.908 & 0.909 & 0.924 & 0.925 & 0.934 & 0.935 & 0.936 & 0.936 & 0.944 & 0.945 & 0.950 & 0.950 \\
\hline 0.09 & 0.896 & 0.897 & 0.915 & 0.915 & 0.926 & 0.927 & 0.927 & 0.928 & 0.937 & 0.937 & 0.943 & 0.944 \\
\hline 0.1 & 0.885 & 0.886 & 0.905 & 0.906 & 0.917 & 0.919 & 0.919 & 0.920 & 0.930 & 0.930 & 0.937 & 0.938 \\
\hline 0.11 & 0.874 & 0.875 & 0.896 & 0.896 & 0.909 & 0.910 & 0.911 & 0.912 & 0.923 & 0.923 & 0.931 & 0.931 \\
\hline 0.17 & 0.807 & 0.808 & 0.838 & 0.840 & 0.858 & 0.860 & 0.861 & 0.862 & 0.879 & 0.880 & 0.891 & 0.892 \\
\hline 0.2 & 0.775 & 0.777 & 0.811 & $\overline{0.812}$ & 0.833 & 0.835 & 0.837 & 0.838 & 0.857 & 0.858 & 0.871 & 0.872 \\
\hline 0.29 & 0.685 & 0.687 & 0.730 & 0.732 & 0.760 & 0.763 & 0.765 & 0.766 & 0.791 & 0.792 & 0.811 & 0.812 \\
\hline 0.4 & 0.589 & 0.591 & 0.641 & 0.642 & 0.676 & 0.679 & 0.681 & 0.683 & 0.715 & 0.716 & 0.739 & 0.741 \\
\hline 0.5 & 0.514 & 0.516 & 0.567 & 0.569 & 0.606 & 0.609 & 0.612 & 0.613 & 0.649 & 0.650 & 0.676 & 0.679 \\
\hline 0.58 & 0.461 & 0.463 & 0.514 & 0.516 & 0.554 & 0.558 & 0.561 & 0.562 & 0.600 & 0.601 & 0.629 & 0.631 \\
\hline 0.75 & 0.365 & 0.367 & 0.417 & 0.419 & 0.457 & 0.461 & 0.464 & 0.465 & 0.504 & 0.506 & 0.536 & 0.538 \\
\hline 0.9 & 0.299 & 0.300 & 0.347 & 0.348 & 0.386 & 0.389 & 0.392 & 0.393 & 0.432 & 0.433 & 0.463 & 0.466 \\
\hline 1 & 0.262 & 0.264 & 0.307 & 0.309 & 0.344 & 0.347 & 0.350 & 0.351 & 0.389 & 0.390 & 0.420 & 0.423 \\
\hline 1.28 & 0.182 & 0.184 & 0.219 & 0.221 & 0.250 & 0.253 & 0.255 & 0.256 & 0.289 & 0.290 & 0.317 & 0.320 \\
\hline 1.4 & 0.156 & 0.158 & 0.190 & 0.191 & 0.218 & 0.220 & 0.223 & 0.224 & 0.254 & 0.255 & 0.280 & 0.282 \\
\hline 1.49 & 0.140 & 0.141 & 0.170 & 0.171 & 0.196 & 0.199 & 0.200 & 0.201 & 0.230 & 0.231 & 0.255 & 0.257 \\
\hline
\end{tabular}

Table B.4:

\begin{tabular}{|c|c|c|c|c|c|c|c|c|c|c|c|c|}
\hline \\
\hline t & 39.82 & 40 & 44.82 & 49.08 & 49.5 & 49.82 & 50 & 54.82 & 55 & 59.08 & 59.5 & 59.82 \\
\hline 0 & 1 & 1 & 1 & 1 & 1 & 1 & 1 & 1 & 1 & 1 & 1 & 1 \\
\hline 0.01 & 994 & 994 & 0.995 & 0.995 & .995 & 0.995 & 0.995 & 0.996 & 0.996 & 0.996 & 0.996 & 0.996 \\
\hline 0.02 & 988 & 988 & 989 & 0.990 & 990 & 990 & 991 & 0.991 & .991 & 0.992 & .992 & .992 \\
\hline 0.03 & 0.982 & 0.982 & .984 & 0.985 & .985 & 0.985 & 0.986 & 0.987 & 0.987 & 0.988 & .988 & 0.988 \\
\hline 0.04 & 0.976 & 0.976 & .979 & 0.980 & .980 & .981 & 0.981 & 0.982 & 0.982 & 0.983 & 0.984 & 0.984 \\
\hline 0.05 & 0.970 & 0.970 & 0.973 & 0.975 & 0.976 & 0.976 & 0.976 & 0.978 & 0.978 & 0.979 & 0.979 & 0.980 \\
\hline 0.06 & 0.963 & 0.964 & 0.967 & 0.970 & 0.970 & 0.970 & 0.971 & 0.973 & 0.973 & 0.975 & 0.975 & 0.975 \\
\hline 0.07 & 0.957 & 0.957 & 0.962 & 0.965 & 0.965 & 0.965 & 0.965 & 0.968 & 0.969 & 0.970 & 0.971 & 0.971 \\
\hline 0.08 & 0.951 & 0.951 & 0.956 & 0.960 & 0.960 & 0.960 & 0.960 & 0.964 & 0.964 & 0.966 & 0.966 & 0.967 \\
\hline 0.09 & 0.944 & 0.944 & 0.950 & 0.954 & 0.955 & 0.955 & 0.955 & 0.959 & 0.959 & 0.962 & 0.962 & 0.962 \\
\hline 0.1 & 0.938 & 0.938 & 0.944 & 0.949 & 0.950 & 0.950 & 0.950 & 0.954 & 0.954 & 0.957 & 0.958 & 0.958 \\
\hline 0.11 & 0.932 & 0.932 & 0.939 & 0.944 & 0.944 & 0.945 & 0.945 & 0.949 & 0.950 & 0.953 & 0.953 & 0.953 \\
\hline 0.17 & 0.893 & 0.893 & 0.904 & 0.911 & 0.912 & 0.913 & 0.913 & 0.920 & 0.921 & 0.926 & 0.926 & 0.926 \\
\hline 0.2 & 873 & 873 & 0.886 & 0.895 & 0.895 & 0.896 & 0.896 & 0.905 & 0.906 & 0.912 & 0.912 & 0.913 \\
\hline 0.29 & 314 & & 832 & 0.845 & 0.846 & 0.847 & 0.847 & 0.860 & 0.860 & 0.869 & 0.870 & 0.870 \\
\hline 0.4 & 742 & 0.743 & 0.765 & 0.782 & 0.784 & 0.785 & 0.786 & 0.802 & 0.803 & 0.814 & 0.816 & 0.816 \\
\hline 0.5 & 0.680 & 0.681 & 0.707 & 0.727 & 0.729 & 0.730 & 0.731 & 0.750 & 0.751 & 0.765 & 0.766 & 0.768 \\
\hline 0.58 & 0.633 & 0.634 & 0.663 & 0.684 & 0.686 & 0.688 & 0.689 & 0.710 & 0.711 & 0.727 & 0.729 & 0.730 \\
\hline 0.75 & 0.541 & 0.542 & 0.573 & 0.598 & 0.600 & 0.602 & 0.603 & 0.628 & 0.628 & 0.647 & 0.649 & 0.651 \\
\hline 0.9 & 0.468 & 0.470 & 0.502 & 0.528 & 0.530 & 0.532 & 0.533 & 0.559 & 0.560 & 0.581 & 0.583 & 0.584 \\
\hline 1 & 425 & & 0.458 & 0.485 & 0.487 & 0.489 & 0.490 & 0.517 & 0.518 & 0.539 & 0.541 & 0.543 \\
\hline 1.28 & 0.322 & & 0.352 & 0.377 & 0.380 & 0.381 & 0.382 & 0.409 & 0.410 & 0.432 & 0.434 & 0.436 \\
\hline 1.4 & 0.284 & 0.285 & 0.314 & 0.338 & 340 & 0.342 & 0.343 & 0.369 & 0.370 & 0.391 & 0.393 & 0.395 \\
\hline 1.49 & 0.259 & 0.260 & 0.287 & 0.311 & 0.313 & 0.315 & 0.316 & 0.341 & 0.342 & 0.363 & 0.365 & 0.367 \\
\hline
\end{tabular}


Table B.5:

\begin{tabular}{|c|c|c|c|c|c|c|c|c|c|c|c|c|}
\hline & \multicolumn{12}{|c|}{$u_{0}$} \\
\hline$t$ & 60 & 64.82 & 65 & 69.08 & 69.5 & 69.82 & 70 & 74.82 & 75 & 79.08 & 79.5 & 79.82 \\
\hline 0 & 1 & 1 & 1 & 1 & 1 & 1 & 1 & 1 & 1 & 1 & 1 & 1 \\
\hline 0.01 & 0.996 & 0.996 & 0.996 & 0.997 & 0.997 & 0.997 & 0.997 & 0.997 & 0.997 & 0.997 & 0.997 & 0.997 \\
\hline 0.02 & 0.992 & 0.993 & 0.993 & 0.993 & 0.993 & 0.993 & 0.993 & 0.993 & 0.993 & 0.994 & 0.994 & 0.994 \\
\hline 0.03 & 0.988 & 0.989 & 0.989 & 0.989 & 0.989 & 0.989 & 0.989 & 0.990 & 0.990 & 0.991 & 0.991 & 0.991 \\
\hline 0.04 & 0.984 & 0.985 & 0.985 & 0.986 & 0.986 & 0.986 & 0.986 & 0.987 & 0.987 & 0.987 & 0.987 & 0.987 \\
\hline 0.05 & 0.980 & 0.981 & 0.981 & 0.982 & 0.982 & 0.982 & 0.982 & 0.983 & 0.983 & 0.984 & 0.984 & 0.984 \\
\hline 0.06 & 0.975 & 0.977 & 0.977 & 0.978 & 0.978 & 0.978 & 0.978 & 0.980 & 0.980 & 0.981 & 0.981 & 0.981 \\
\hline 0.07 & 0.971 & 0.973 & 0.973 & 0.974 & 0.975 & 0.975 & 0.975 & 0.976 & 0.976 & 0.978 & 0.978 & 0.978 \\
\hline 0.08 & 0.967 & 0.969 & 0.969 & 0.971 & 0.971 & 0.971 & 0.971 & 0.973 & 0.973 & 0.974 & 0.974 & 0.974 \\
\hline 0.09 & 0.962 & 0.965 & 0.965 & 0.967 & 0.967 & 0.967 & 0.967 & 0.969 & 0.969 & 0.971 & 0.971 & 0.971 \\
\hline 0.1 & 0.958 & 0.961 & 0.961 & 0.963 & 0.963 & 0.963 & 0.963 & 0.966 & 0.966 & 0.967 & 0.968 & 0.968 \\
\hline 0.11 & 0.954 & 0.957 & 0.957 & 0.959 & 0.959 & 0.960 & 0.960 & 0.962 & 0.962 & 0.964 & 0.964 & 0.964 \\
\hline 0.17 & 0.927 & 0.932 & 0.932 & 0.936 & 0.936 & 0.936 & 0.936 & 0.940 & 0.941 & 0.944 & 0.944 & 0.944 \\
\hline 0.2 & 0.913 & 0.919 & 0.919 & 0.924 & 0.924 & 0.924 & 0.925 & 0.929 & 0.929 & 0.933 & 0.933 & 0.934 \\
\hline 0.29 & 0.871 & 0.879 & 0.880 & 0.886 & 0.887 & 0.887 & 0.888 & 0.895 & 0.895 & 0.900 & 0.901 & 0.901 \\
\hline 0.4 & 0.817 & 0.829 & 0.830 & 0.838 & 0.839 & 0.840 & 0.840 & 0.850 & 0.850 & 0.857 & 0.858 & 0.858 \\
\hline 0.5 & 0.768 & 0.783 & 0.784 & 0.795 & 0.796 & 0.797 & 0.797 & 0.809 & 0.809 & 0.818 & 0.819 & 0.819 \\
\hline 0.58 & 0.731 & 0.747 & 0.748 & 0.761 & 0.762 & 0.763 & 0.763 & 0.776 & 0.777 & 0.787 & 0.788 & 0.789 \\
\hline 0.75 & 0.651 & 0.672 & 0.672 & 0.688 & 0.689 & 0.690 & 0.691 & 0.707 & 0.708 & 0.721 & 0.722 & 0.723 \\
\hline 0.9 & 0.585 & 0.607 & 0.608 & 0.625 & 0.627 & 0.628 & 0.629 & 0.647 & 0.648 & 0.663 & 0.664 & 0.665 \\
\hline 1 & 0.544 & 0.567 & 0.568 & 0.586 & 0.588 & 0.589 & 0.589 & 0.609 & 0.610 & 0.625 & 0.626 & 0.628 \\
\hline 1.28 & 0.437 & 0.460 & 0.461 & 0.481 & 0.482 & 0.484 & 0.485 & 0.506 & 0.506 & 0.523 & 0.525 & 0.526 \\
\hline 1.4 & 0.396 & 0.419 & 0.420 & 0.440 & 0.442 & 0.443 & 0.444 & 0.465 & 0.466 & 0.483 & 0.485 & 0.486 \\
\hline 1.49 & 0.367 & 0.391 & 0.391 & 0.411 & 0.413 & 0.414 & 0.415 & 0.437 & 0.437 & 0.455 & 0.457 & 0.458 \\
\hline
\end{tabular}

Table B.6:

\begin{tabular}{lccccccccccc}
\hline $\mathbf{t}$ & 84.82 & 85 & 89.08 & 89.5 & 89.82 & 90 & 94.82 & 99.08 & 99.5 & 99.82 & 100 \\
\hline $\mathbf{0}$ & 1 & 1 & 1 & 1 & 1 & 1 & 1 & 1 & 1 & 1 & 1 \\
\hline $\mathbf{0 . 0 1}$ & 0.997 & 0.997 & 0.997 & 0.997 & 0.997 & 0.997 & 0.997 & 0.998 & 0.998 & 0.998 & 0.998 \\
\hline $\mathbf{0 . 0 2}$ & 0.994 & 0.994 & 0.994 & 0.994 & 0.995 & 0.995 & 0.995 & 0.995 & 0.995 & 0.995 & 0.995 \\
\hline $\mathbf{0 . 0 3}$ & 0.991 & 0.991 & 0.992 & 0.992 & 0.992 & 0.992 & 0.992 & 0.992 & 0.992 & 0.992 & 0.992 \\
\hline $\mathbf{0 . 0 4}$ & 0.988 & 0.988 & 0.989 & 0.989 & 0.989 & 0.989 & 0.989 & 0.990 & 0.990 & 0.990 & 0.990 \\
\hline $\mathbf{0 . 0 5}$ & 0.985 & 0.985 & 0.986 & 0.986 & 0.986 & 0.986 & 0.987 & 0.987 & 0.987 & 0.987 & 0.987 \\
\hline $\mathbf{0 . 0 6}$ & 0.982 & 0.982 & 0.983 & 0.983 & 0.983 & 0.983 & 0.984 & 0.985 & 0.985 & 0.985 & 0.985 \\
\hline $\mathbf{0 . 0 7}$ & 0.979 & 0.979 & 0.980 & 0.980 & 0.980 & 0.980 & 0.981 & 0.982 & 0.982 & 0.982 & 0.982 \\
\hline $\mathbf{0 . 0 8}$ & 0.976 & 0.976 & 0.977 & 0.977 & 0.977 & 0.977 & 0.978 & 0.979 & 0.979 & 0.979 & 0.980 \\
\hline $\mathbf{0 . 0 9}$ & 0.973 & 0.973 & 0.974 & 0.974 & 0.974 & 0.974 & 0.976 & 0.977 & 0.977 & 0.977 & 0.977 \\
\hline $\mathbf{0 . 1}$ & 0.970 & 0.970 & 0.971 & 0.971 & 0.971 & 0.971 & 0.973 & 0.974 & 0.974 & 0.974 & 0.974 \\
\hline $\mathbf{0 . 1 1}$ & 0.967 & 0.967 & 0.968 & 0.968 & 0.968 & 0.968 & 0.970 & 0.971 & 0.971 & 0.971 & 0.972 \\
\hline $\mathbf{0 . 1 7}$ & 0.947 & 0.948 & 0.950 & 0.950 & 0.950 & 0.950 & 0.953 & 0.955 & 0.955 & 0.955 & 0.955 \\
\hline $\mathbf{0 . 2}$ & 0.938 & 0.938 & 0.941 & 0.941 & 0.941 & 0.941 & 0.944 & 0.946 & 0.946 & 0.947 & 0.947 \\
\hline $\mathbf{0 . 2 9}$ & 0.907 & 0.907 & 0.911 & 0.911 & 0.912 & 0.912 & 0.916 & 0.920 & 0.920 & 0.920 & 0.921 \\
\hline $\mathbf{0 . 4}$ & 0.866 & 0.867 & 0.873 & 0.873 & 0.874 & 0.874 & 0.880 & 0.885 & 0.885 & 0.886 & 0.886 \\
\hline $\mathbf{0 . 5}$ & 0.829 & 0.829 & 0.837 & 0.838 & 0.838 & 0.839 & 0.847 & 0.853 & 0.853 & 0.854 & 0.854 \\
\hline $\mathbf{0 . 5 8}$ & 0.800 & 0.800 & 0.809 & 0.810 & 0.810 & 0.811 & 0.820 & 0.827 & 0.828 & 0.828 & 0.829 \\
\hline $\mathbf{0 . 7 5}$ & 0.737 & 0.737 & 0.748 & 0.749 & 0.750 & 0.750 & 0.762 & 0.771 & 0.772 & 0.773 & 0.773 \\
\hline $\mathbf{0 . 9}$ & 0.681 & 0.682 & 0.694 & 0.695 & 0.696 & 0.696 & 0.709 & 0.720 & 0.721 & 0.722 & 0.722 \\
\hline $\mathbf{1}$ & 0.645 & 0.645 & 0.658 & 0.660 & 0.661 & 0.661 & 0.675 & 0.687 & 0.688 & 0.688 & 0.689 \\
\hline $\mathbf{1 . 2 8}$ & 0.545 & 0.546 & 0.560 & 0.562 & 0.563 & 0.563 & 0.580 & 0.594 & 0.595 & 0.596 & 0.596 \\
\hline $\mathbf{1 . 4}$ & 0.505 & 0.506 & 0.521 & 0.523 & 0.524 & 0.525 & 0.541 & 0.555 & 0.557 & 0.558 & 0.558 \\
\hline $\mathbf{1 . 4 9}$ & 0.477 & 0.478 & 0.493 & 0.495 & 0.496 & 0.496 & 0.513 & 0.528 & 0.529 & 0.530 & 0.531 \\
\hline & & & & & & & & & & &
\end{tabular}




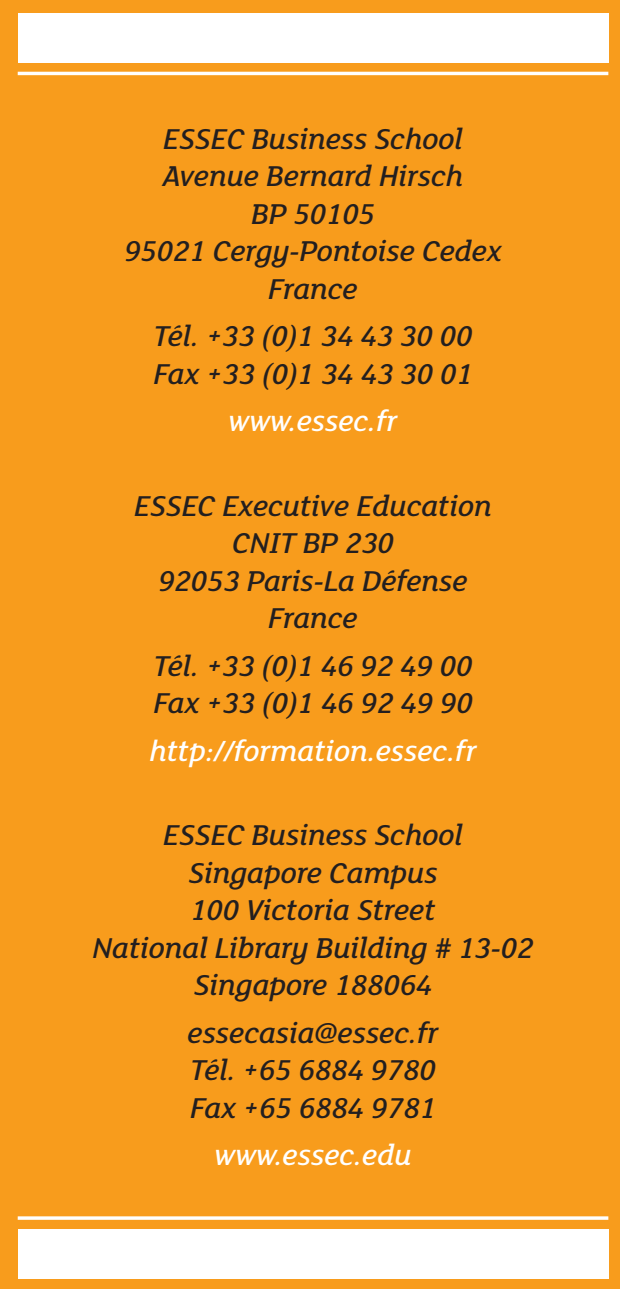

\section{Informations}

+33 (0)134433091

blanchard@essec.fr

research.center@essec.fr

www.essec.fr

\section{ISSIC

